UNIVERSIDADE DE SÃO PAULO

INSTITUTO DE FÍSICA DE SÃO CARLOS

JHONNY RICHARD HUAMANI CHAVIGURI

Transição de fase quântica de sistema $2 D$ em rede de vórtices

São Carlos

2016 

JHONNY RICHARD HUAMANI CHAVIGURI

\title{
Transição de fase quântica de sistema $2 D$ em rede de vórtices
}

\author{
Dissertação apresentada ao programa de Pós- \\ Graduação em Física do Instituto de Física de \\ São Carlos da Universidade de São Paulo, para \\ obtenção do título de Mestre em Ciências. \\ Área de concentração: Física Básica \\ Orientadora: Dra. Mônica Andrioli Caracanhas
}

\author{
Versão Corrigida \\ (Versão original disponivel na Unidade que aloja o Programa)
}

São Carlos

2016 
AUTORIZO A REPRODUÇÃO E DIVULGAÇÃO TOTAL OU PARCIAL DESTE TRABALHO, POR QUALQÜER MEIO CONVENCIONAL OU ELETRÔNICO PARA FINS DE ESTUDO E PESQUISA, DESDE QUE CITADA A FONTE.

Ficha catalográfica revisada pelo Serviço de Biblioteca e Informação do IFSC, com os dados fornecidos pelo(a) autor(a)

Huamani Chaviguri, Jhonny Richard

Transição de fase quântica de sistema 2D em rede de vórtices / Jhonny Richard Huamani Chaviguri;

orientadora Mônica Andrioli Caracanhas - versão corrigida -- São Carlos, 2016.

$101 \mathrm{p}$.

Dissertação (Mestrado - Programa de Pós-Graduação em Física Básica) -- Instituto de Física de São Carlos, Universidade de São Paulo, 2016.

1. Condensado de Bose-Einstein. 2. Rede de vórtices. 3. Transição de fase quântica. I. Caracanhas, Mônica Andrioli, orient. II. Título. 
À minha mãe, Saturnina. 



\section{Agradecimentos}

Primeiramente, agradeço à minha família por todo seu apoio, em especial ao meus tios-pais Alberto, Nina e Marcelina, meus primos Carlos, Dávid, Meri e Joel, minhas irmãs Eli e Katty, e à minha irmãzinha Fiorela.

Agradeço ao meu grande amigo Franklin por sua inestimável ajuda durante minha estadia em São Carlos, igualmente para sua esposa Anne. Também para os novos amigos que conheci no grupo de Óptica: Amilson, Pedro, Edwin, Emmanuel, Thiago, e para os que estou esquecendo. Para os professores Reginaldo Napolitano, Carlos Egues, Frederico Brito e Leonardo Maia por seus ensinamentos. Para os funcionários da biblioteca, em especial para a senhora Maria Neusa por sua diligência. Para Ítalo da gráfica. Para meus camaradas da república: Sergão, Marcão, Victor, Luiz, Rafael, Lito e Felipe, para todos eles muito obrigado por fazer minha estadia no Brasil muito mais que interessante.

Agradeço ao Instituto de Física de São Carlos, pela oportunidade de realização do curso de mestrado. Às agências de fomento à pesquisa CAPES, FAPESP e, particularmente, à CNPq de quem fui bolsista neste mestrado.

Finalmente, agradeço ao Prof. Vanderlei por me aceitar, fazendo um pequeno buraco, ser parte de seu numeroso grupo de pesquisadores no grupo de Óptica, e à minha orientadora Mônica, por sua altíssima paciência para me compreender, tolerar (espero) e fornecer as pautas precisas no momento certo e, obviamente, por sua inestimável ajuda para que esse trabalho chegue a bom porto durante esses dois anos. 



\section{Resumo}

HUAMANI CHAVIGURI, J. R. Transição de fase quântica de sistema $2 \mathrm{D}$ em rede de vórtices. 2016. 101 p. Dissertação (Mestrado em Ciências) - Instituto de Física de São Carlos, Universidade de São Paulo, São Carlos, 2016.

Neste trabalho estudamos um sistema bidimensional composto de duas espécies atômicas condensadas, uma delas contendo uma rede de vórtices. Analogamente ao modelo desenvolvido para tratar de átomos ultrafrios em redes ópticas, mapeamos o Hamiltoniano do nosso sistema com o Hamiltoniano do modelo Bose-Hubbard (BH), com o potencial periódico da rede advindo da interação de campo médio entre as duas espécies. A variação do comprimento de espalhamento atômico permite alterar as propriedades do potencial confinante, com a indução da transição de fase quântica na espécie aprisionada nos vórtices. O novo aspecto trazido pela rede de vórtices advém dos seus modos de excitação de baixa energia, os modos de Tkachenko. Consideramos os efeitos da dinâmica própria desse potencial sobre a espécie aprisonada através de um modelo BH efetivo, com novos valores para interação local e tunelamento, além de um termo adicional de interação de longo alcance, mediada pelos modos da rede. Além de complementar os estudos com redes ópticas estáticas, a proposta teórica desenvolvida apresenta grande viabilidade experimental no contexto das técnicas atuais para manipulação de átomos ultrafrios.

Palavras-chaves: Condensação de Bose-Einstein. Rede de vórtices. Transição de fase quântica. 



\section{Abstract}

HUAMANI CHAVIGURI, J. R. Quantum phase transition of 2D system in a vortex lattice. 2016. 101 p. Dissertação (Mestrado em Ciências) - Instituto de Física de São Carlos, Universidade de São Paulo, São Carlos, 2016.

In this work we consider a two dimensional system composed of two condensed atomic species, one containing a vortex lattice. Analogously to the model used to describe ultracold atoms in optical lattices, we mapped our system Hamiltonian in the Hamiltonian of the Bose-Hubbard (BH) model, with the periodic lattice potential arising from the meanfield interaction between the two species. The variation of the atomic scattering length allow us to change the properties of the confining potential, to induce the quantum phase transition in the species trapped in the vortices. The new aspect brought by the vortex lattice comes with its low energy normal modes, the Tkachenko modes. We considered the effects of such dynamic potential over the confined species thought an effective BH model, with new values for the local interaction and tunneling parameters, besides an additional long-range interaction term mediated by the lattice modes. Our theoretical proposal goes beyond the studies with static optical lattice. Additionally, it has great feasibility in the current context of ultra-cold atoms experimental techniques.

Keywords: Bose-Einstein condensation. Vortex lattice. Quantum phase transition. 



\section{Lista de figuras}

Figura 1 - Etapas experimentais da criação do BEC. (a) Formação da nuvem atômica térmica antes de atingir a temperatura crítica $\left(T>T_{c}\right)$. (b) Comportamento bimodal da nuvem atômica compostas pela componente do BEC (pico) e outra associada aos átomos térmicos residuais $\left(T<T_{c}\right)$. (c) Obtenção do BEC puro $\left(T \ll T_{c}\right)$, onde a presença da nuvem residual é quase nula. $N_{B E C} \sim 1.5 \times 10^{6}$. Escala de cor indica maior densidade atômica. . . . . . . . . . . . . . . . .

Figura 2 - Etapas que atravessa o gás ideal de bósons diluído até atingir o con-

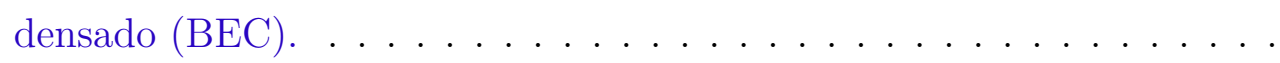

Figura 3 - Perfil de densidade atômica do regime de TF e solução numérica da GPE estacionária, Eq. (2.15). O deslocamento da GPE com respeito ao perfil parabólico de TF é da ordem do healing length. Como $N a_{s} / a_{o h} \sim$ 100, o regime de TF é uma boa aproximação para descrever o BEC. . .

Figura 4 - Imagens experimentais desde 1 até um arranjo de 130 vórtices. Acima: Formação desde 1 até 13 vórtices. O condensado tem seu formato variando de um cigar-shape para um pancake-shape a medida que adquire maior número de vórtices. Abaixo: Cristalização dos vórtices em rede triangular para diferentes tempos na armadilha; observam-se (A) 16, (B) 32, (C) 80 e (D) 130 vórtices. . . . . . . . . . . . . . .

Figura 5 - Superfluído aprisionado em armadilha cilíndrica com frequência angular $\Omega=\Omega \hat{z}$. Acima de um valor crítico de $\Omega$ temos o aparecimento das excitações de vórtice no superfluido. $R$ e $v$ são o raio do cilindro e velocidade do superfluido. . . . . . . . . . . . . . . .

Figura 6 - (a) BEC quase 2D composto de um arranjo de vórtices. (b) Vórtice unitário rodeado pelo magnetic length a. O núcleo do vórtice é da ordem do healing length, $\xi \ldots \ldots \ldots \ldots \ldots \ldots$ 
Figura 7 - Diferentes configurações espaciais da rede óptica. Esquerda: (a) 1D, (b) $2 D$, , c $3 D$. Direita: Configurações geradas quando se mudam as direções dos lasers em $2 D$ tais como hexagonal e triangular. . . . . . . .

Figura 8 - Redes rasa e profunda obtidas variando externamente a intensidade dos feixes de lasers. . . . . . . . . . . . . . . . . . .

Figura 9 - Bandas de energia (circunscritos no esquema da zona reduzida) de um átomo com a profundidade do potencial óptico $V_{0}$ dada em termos da energia de recuo $E_{R}$. (a) $V_{0}=0$ átomo livre. (b) $V_{0}=4 E_{R}$ rede rasa.

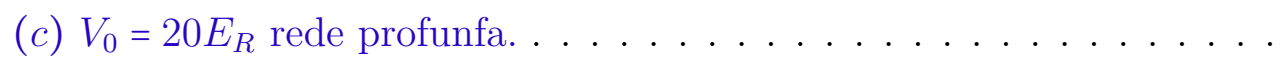

Figura 10 - Etapas atravessadas pela amostra aprisionada até atingir a TFQ. As imagens se referem à distribuicao dos átomos no espaço dos momentos. Os valores de $V_{0}=(a): 0 E_{R},(b): 3 E_{R},(c): 7 E_{R},(d): 10 E_{R}$, $(e): 13 E_{R},(f): 14 E_{R},(g): 16 E_{R},(h): 20 E_{R}$ e $(i): 22 E_{R} . \ldots$.

Figura 11 - Rede óptica com fator de preenchimento $\nu=1$. (a) Fase superfluida, os átomos estão delocalizados. (b) Fase isolante de Mott, os átomos estão localizados. . . . . . . . . . . . . . . . . . . 46

Figura 12 - Gráfico da equação (4.37) com $\bar{\mu}=\mu / z J$ e $\bar{U}=U / z J$. A área correspondente à fase de isolante de Mott varia de acordo com o número de ocupação dos sítios da rede. . . . . . . . . . . . . . . . . . . . 51

Figura 13 - Imagem de absorção (perfil de densidade) da espécie $A$ composta de um arranjo triangular de vórtices. . . . . . . . . . . . . . 55

Figura 14 - Variação do potencial local do sítio (vórtices) nos limites extremos da

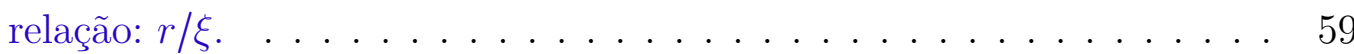

Figura 15 - Parâmetro de rede $d$, magnetic length a, healing length $\xi$ e o comprimento $l_{0}$ do perfil gaussiano da função de Wannier. . . . . . . . . . . . . .

Figura 16 - Diagrama de fases para $n=1$. O lóbulo interno (vermelho tracejada) foi feita pela aproximação de mean-field, enquanto que o lóbulo exterior (azul) foi obtida pela TC através da equação (5.32) usando $z=6$. . . .

Figura 17 - Valores críticos do espalhamento $a_{B}$ para as misturas: ${ }^{23} \mathrm{Na}-{ }^{87} \mathrm{Rb}$ à esquerda e ${ }^{87} \mathrm{Rb}-{ }^{133} \mathrm{Cs}$ à direita. . . . . . . . . . . . . . . 66 
Figura 18 - Valores críticos do espalhamento $a_{A B}$ para as misturas: ${ }^{23} \mathrm{Na}-{ }^{87} \mathrm{Rb}$ à esquerda e ${ }^{87} \mathrm{Rb}-{ }^{41} \mathrm{~K}$ à direita. . . . . . . . . . . . . . . . 67

Figura 19 - Valores críticos do espalhamento $a_{A B}$ para as misturas: ${ }^{87} \mathrm{Rb}-{ }^{133} \mathrm{Cs}$ à esquerda e ${ }^{23} \mathrm{Na}-{ }^{41} \mathrm{~K}$ à direita. . . . . . . . . . . . . . . . . . 67

Figura 20 - Modos de Tkachenko observados no experimento do JILA no regime de $\mathrm{TF}$ com $\Gamma_{L L L}=0.6 \ldots \ldots \ldots \ldots \ldots$

Figura 21 - Diagrama de fases para o sistema estendido, caso $\tilde{\mathbf{V}} \ll \tilde{\mathbf{U}}$. Lóbulo com preenchimento unitário: linha tracejada representando cálculo MF com $a=0$; em vermelho $a=0.01$, em marrom $a=0.05$, em preto, $a=0.1 \mathrm{em}$

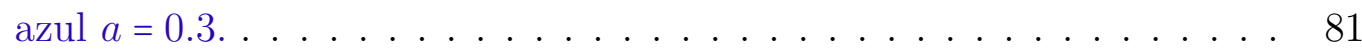





\section{Lista de tabelas}

Tabela 1 - Valores experimentais para os espalhamentos da onda-s $a_{s}$ e massa $m$ para distintos átomos alcalinos. $a_{0}$ é o raio de Bohr . . . . . . . . . 65

Tabela 2 - Valores aproximados para o espalhamento $a_{B}$ com fator de Landau $\Gamma_{L L L}=0.6$ para a mistura de espécies diferentes. $l_{z}$ foi computado empregando $\omega_{z}=200 \times 2 \pi \mathrm{rad} / \mathrm{s} . \ldots \ldots \ldots 6$

Tabela 3 - Valores aproximados para o espalhamento $a_{A B}$ com fator de Landau $\Gamma_{L L L}=0.6$ para a mistura de espécies diferentes. $l_{z}$ foi computado empregando $\omega_{z}=200 \times 2 \pi \mathrm{rad} / \mathrm{s} \ldots \ldots \ldots \ldots \ldots$. . . . . . . . . 67 



\section{Sumário}

INTRODUÇÃO . . . . . . . . . . . . . . . . . 19

NOÇÕES PRELIMINARES . . . . . . . . . . . . . . . . . 21

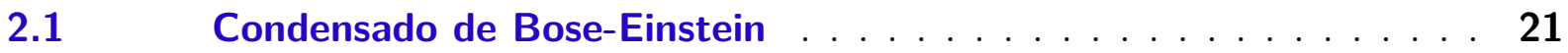

2.1.1 BEC não interagente: Gás ideal de Bose . . . . . . . . . . . . . . 24

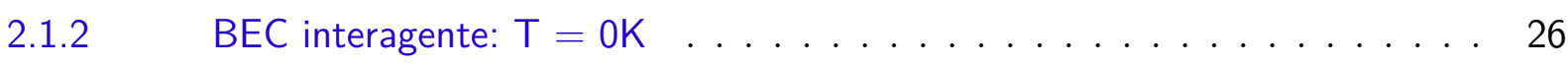

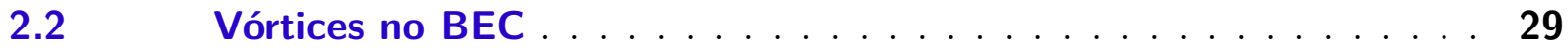

2.2.1 Vórtice unitário: Quantização da circulação . . . . . . . . . . . . . 30

2.2.2 BEC em rotação: Rede de Vórtices . . . . . . . . . . . . . . . 31

3 ÁtOMOS UltRAFRIOS EM REDES ÓPTICAS . . . . . . . . . . . 33

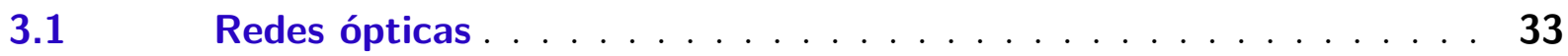

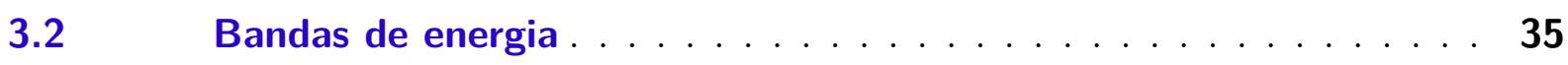

3.2.1 Rede profunda: Aproximação de tight-binding . . . . . . . . . . 36

3.2.2 Funções de Wannier . . . . . . . . . . . . . . . . . . . 37

$3.3 \quad$ BEC em redes ópticas $\ldots \ldots \ldots \ldots \ldots$

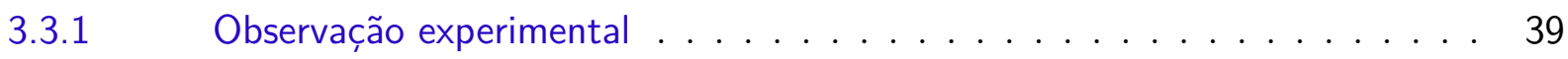

4 MODELO DE BOSE-HUBBARD $\ldots \ldots \ldots \ldots \ldots \ldots$

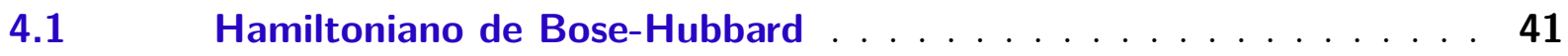

$4.1 .1 \quad$ Aproximação gaussiana . . . . . . . . . . . . 43

$4.1 .2 \quad$ Equação de Mathieu . . . . . . . . . . . . . . . . . . 45

$4.2 \quad$ Transição de fase quântica . . . . . . . . . . . . . . 46

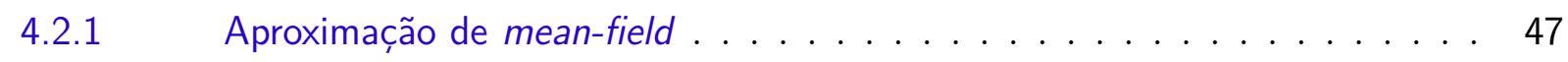

4.2.2 Teoria de perturbações de Rayleigh-Schrödinger . . . . . . . . . . . . 49

$4.2 .3 \quad$ Diagrama de fases . . . . . . . . . . . . . . . 50 
$5.1 \quad$ Regimes do estudo $\ldots \ldots \ldots \ldots \ldots$

$5.1 .1 \quad$ Características das espécies para $T \approx 0 K \ldots \ldots \ldots \ldots$

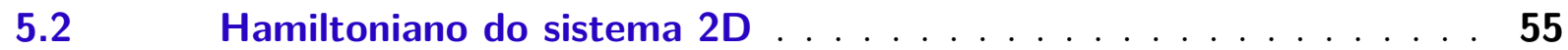

$5.2 .1 \quad$ Espécie B: Limite de tight-binding . . . . . . . . . . . . . 57

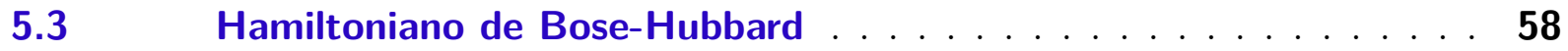

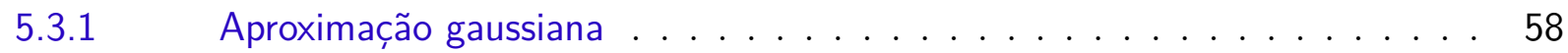

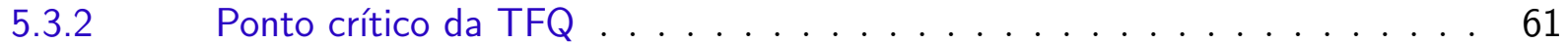

$5.4 \quad$ Transição de fase quântica . . . . . . . . . . . . 62

5.4.1 Aproximação de Teoria de Campos . . . . . . . . . . . . . . 62

$5.4 .2 \quad$ Diagrama de Fases . . . . . . . . . . . . . . . . . . 64

6 MODOS VIBRACIONAIS DA REDE DE VÓRTICES . . . . . . . 69

$6.1 \quad$ Espécie $A$ : Transformação de Bogoliubov . . . . . . . . . . . . 69

$6.1 .1 \quad$ Expansão dos modos vibracionais . . . . . . . . . . . . . 71

6.1.2 Hamiltoniano de Bogoliubov . . . . . . . . . . . . . . . . . 72

$6.2 \quad$ Interação entre as espécies: $A-B \ldots \ldots \ldots \ldots$

$6.3 \quad$ Hamiltoniano total do sistema estendido $\ldots \ldots \ldots \ldots$

$6.3 .1 \quad$ Transformação unitária . . . . . . . . . . . . . . 76

6.3.2 Hamiltoniano de Bose-Hubbard estendido: $\alpha$ particular . . . . . . . . . 77

6.3.3 Transição de fase quântica: Aproximação de MF . . . . . . . . . . . . . . . 79

$7 \quad$ CONCLUSÕES $\ldots \ldots \ldots \ldots$

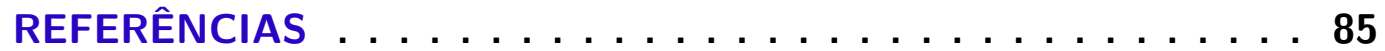

APÊNDICE A - HAMILTONIANO DE BOSE-HUBBARD ESTENDIDO: $\alpha$ GERAL . . . . . . . . . . . . 93

APÊNDICE B - PARÂMETROS ASSOCIADOS AOS MODOS . . 95

APÊNDICE C - APROXIMAÇÃO DE MF DA TFQ . . . . . . . 99 


\section{Introdução}

Os condensados com misturas de bósons possuem rica física nova a ser explorada. Existem importantes experimentos nesse campo, que produzem condensados de BoseEinstein (BEC) utilizando diferentes espécies hiperfinas do átomo de rubídio ${ }^{87} \mathrm{Rb}(1-3)$, onde as propriedades estáticas da mistura, a fase relativa entre os condensados e sua dinâmica foram investigadas. Esse mesmo grupo experimental foi também pioneiro na nucleação de vórtices nesse sistema composto (3), produzindo inclusive a superposição de duas redes de vórtices. (4) A possibilidade de variar o comprimento de espalhamento atômico é uma importante ferramenta para lidar com os condensados de duas espécies. Nos experimentos $(5,6)$, foram utilizadas diferentes espécies atômicas, precisamente átomos de rubídio ${ }^{87} \mathrm{Rb}$ e potássio ${ }^{41} \mathrm{~K}$, para produzir um condensado de duas componentes através da técnica do resfriamento mútuo (Sympathetic cooling). Apesar das diferentes armadilhas vistas por cada uma das espécies (diferentes massas envolvidas), mesmo uma pequena sobreposição das nuvens condensadas já trouxeram efeitos observáveis devido à interação. $(7,8)$ Importantes resultados obtidos com esses experimentos se baseiam no controle da interação entre as espécies por meio da técnica de ressonância de Feshbach. $(8,9)$

Tendo como base esses experimentos e a teoria relacionada a eles, nesse trabalho analisa-se uma mistura de superfluidos atômicos. O modelo teórico proposto baseia-se na possibilidade de aprisionar uma espécie superfluida bastante diluída (espécie $B$ ) nos vórtices de uma segunda espécie (espécie $A$ ), com densidade de átomos bastante superior à primeira. A espécie $A$ consiste de um condensado com rede de vórtices que interagirá com um outro superfluido $B$, ambos em duas dimensões espaciais. As propriedades do sistema resultante foram analisadas através do modelo Bose-Hubbard, com a proposição de um experimento para caracterizar a transição de fase quântica da espécie aprisionada, por meio do controle da sua seção de choque de espalhamento.

Analisa-se também as transições de fase da espécie $B$ aprisionada na rede de vórtices da espécie $A$. O aprisionamento de átomos ultrafrios em redes ópticas aumentou 
o poder dessa área de pesquisa em simular modelos de matéria condensada. $(10,11)$ A variação das propriedades do potencial óptico, frequência e intensidade dos lasers envolvidos na criação da rede, permite controlar as taxas de tunelamento e a interação dos átomos aprisionados. Esse controle das energias cinética e potencial dos átomos na rede possibilitou a transição entre os estados superfluido e isolante de Mott da amostra aprisionada, com inúmeros trabalhos teóricos e experimentais caracterizando o diagrama de fases. $(11-13)$

Com a proposta da rede de vórtices $(14,15)$ pretende-se estudar um sistema de rede dinâmica, que possui vibrações intrínsecas dadas pelos modos de Tkachenko (16-18), e que também apresenta grande interesse para realização experimental, pois se enquadra no cenário das mais recentes técnicas desenvolvidas para manipulação dos átomos frios. Dentre esses avanços experimentais, destaca-se a produção das misturas de duas espécies condensadas através da técnica do resfriamento mútuo, e também a possibilidade de gerar vórtices seletivamente em uma das espécies da mistura, através da manipulação dos seus estados hiperfinos com os campos de Calibre artificiais. (19) Para completar, tem-se ainda uma técnica auxiliar para o controle da interação entre os átomos, a ressonância de Feshbach (20), que será peça chave para variar o potencial da rede, o qual advém da interação repulsiva entre os átomos da rede e os da espécie aprisionada.

A dissertação será apresentada nas seguintes etapas: o capítulo 2 descreve o formalismo matemático para os átomos ultrafrios relevantes na obtenção do BEC; analisa-se a dinâmica desse BEC e as suas excitações de vórtices. No capítulo 3 são analisadas as características do sistema de átomos ultrafrios aprisionados em rede óptica, e a sua relação com a física do estado sólido através das funções de onda de Bloch. No capítulo 4, aplica-se o modelo de Bose-Hubbard com a aproximação de tight binding para bósons na rede óptica. No capítulo 5 a primeira parte do modelo teórico proposto é desenvolvida, com o tratamento "estático" para rede de vórtices. O capítulo 6 complementa essa primeira etapa do referido modelo, introduzindo a "dinâmica" da rede através dos modos de Tkachenko. Os principais resultados do modelo teórico proposto são sumarizados no capítulo final com as conclusões. 


\section{Noções preliminares}

Este capítulo apresenta um breve resumo dos conceitos básicos e a formulação matemática elementar associada ao condensado de Bose-Einstein. Também se analisa seu comportamento sob excitações externas, focado na formação dos vórtices. Esse capítulo está fundamentado com base nos livros-texto. $(20,21)$

\subsection{Condensado de Bose-Einstein}

Um grande acontecimento ocorrido na última década do século $\mathbf{X X}$ foi a obtenção experimental do condensado do gás de Bose em gases alcalinos $\left({ }^{7} \mathrm{Li},{ }^{23} \mathrm{Na},{ }^{87} \mathrm{Rb}\right)$.

Historicamente, no ano 1924, S. N. Bose (22) elaborou uma descrição estatística dos "quanta" de luz (fótons). Em 1925, A. Einstein (23) empregou essa descrição para estudar o menor estado de energia a baixas temperaturas de um gás de Bose não interagente, predizendo o chamado condensado de Bose-Einstein. Anos depois, em 1938, P. L. Kapitza (24), J. F. Allen e A. D. Misener (25) reportaram independentemente seus descobrimentos sobre a superfluidez do He líquido (conhecido como He tipo 2) e no mesmo ano F. London (26) propõe que o BEC possui características de superfluidez.

Por volta de 1941, L. D. Landau (27) desenvolveu a teoria dos superfluidos do He líquido (interações atômicas fortes) empregando o modelo dos dois fluidos (fluido clássico e superfluido quântico). Nessa teoria, Landau introduz o conceito de parâmetro de ordem (valor esperado). Em 1947, N. N. Bogoliubov (28) formulou a teoria microscópica do gás de Bose fracamente interagente. Nessa teoria ele introduz o conceito da flutuação (surge como uma manifestação das interações fracas), com o estado fundamental do sistema podendo ser descrito como um parâmetro de ordem somado às flutuações. Essa teoria é adequada para descrever o BEC obtido nos experimentos, posto que os gases alcalinos a 
baixas temperaturas são muito diluídos e interagem fracamente.

No ano 1961, E. P. Gross (29) e L. P. Pitaevskii (30) derivam independentemente uma equação de Schrödinger não linear utilizando argumentos da teoria de mean-field. Essa equação será denominada como equação de Gross-Pitaevskii (GPE). Em torno dos anos de 1986 e 1988, C. Cohen-Tannoudji (31), W. D. Phillips (32) e S. Chu (33) desenvolvem técnicas óptico-magnéticas de resfriamento em experimentos de átomos frios. Finalmente, em 1995, E. A. Cornell e C. E. Wieman (34), W. Ketterle (35) e R. G. Hulet (36) conseguiram observar experimentalmente o BEC em gases alcalinos confinados de ${ }^{87} \mathrm{Rb},{ }^{23} \mathrm{Na}$ e ${ }^{7} \mathrm{Li}$ respectivamente. Usualmente esses gases diluídos e ultrafrios possuem densidade da ordem de $10^{-14} \mathrm{~cm}^{-3}$ e temperaturas na escala de $10^{-9} \mathrm{~K}$.
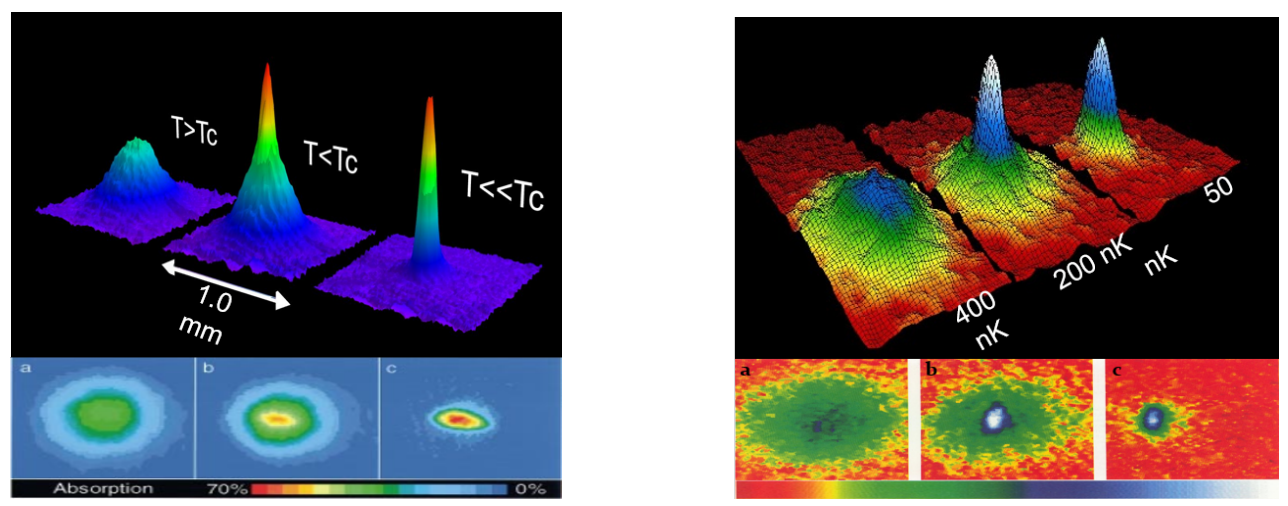

Figura 1 - Etapas experimentais da criação do BEC. (a) Formação da nuvem atômica térmica antes de atingir a temperatura crítica $\left(T>T_{c}\right)$. (b) Comportamento bimodal da nuvem atômica compostas pela componente do BEC (pico) e outra associada aos átomos térmicos residuais $\left(T<T_{c}\right)$. (c) Obtenção do BEC puro $\left(T \ll T_{c}\right)$, onde a presença da nuvem residual é quase nula. $N_{B E C} \sim 1.5 \times 10^{6}$. Escala de cor indica maior densidade atômica.

Fonte: KETTERLE; PRITCHARD (37) à direita e CORNELL (38) à esquerda.

- Definição do BEC: É o estado da matéria no qual todos os átomos em conjunto ocupam um único estado quântico (menor estado de energia). Esse estado é descrito por meio de uma única função de onda macroscópica denominada função de onda do condensado, que está composta de muitas funções de ondas individuais superpostas.

• Condição para obter BEC: O gás deve ser diluído (distância média interatômica, $d$, é muito maior ao comprimento de espalhamento da onda-s, $a_{s}$ )

$$
n\left|a_{s}\right|^{3} \ll 1 \quad \text { com } n \sim d^{-3}
$$


onde $n$ representa a densidade espacial dos átomos. O sinal de $a_{s}$ representa natureza do potencial de contato e influência diretamente na formação do BEC [Condensado com $a_{s}>0$ (interação repulsiva) $(34,35)$ e com $a_{s}<0$ (interação atrativa) (36)].

O processo de formação do BEC ideal é esquematizado na Figura ??. Resumindo as etapas: considere um gás bosónico diluído com $N$ átomos. (a) Os átomos a altas temperaturas comportam-se como pontos materiais clássicos (bolas de bilhar) que são caracterizados pela velocidade $(v)$ e densidade atômica $\left(n \sim d^{-3}\right)$. Nessa etapa os átomos são governados pelas leis da mecânica clássica. (b) Quando a temperatura começa descer, os átomos mostram sua natureza ondulatória. Cada ponto material torna-se um pacote de onda, cuja dimensão fica especificado pelo seu comprimento de onda. Essa dimensão é o comprimento de onda térmico de de Broglie $\left[\lambda_{d B}=h /\left(2 \pi m k_{B} T\right)^{1 / 2}\right.$, onde $m$ é a massa do átomo]. A partir dessa etapa os átomos passam a ser governados pelas leis da mecânica quântica. (c) No momento em que os átomos atingem a temperatura crítica $\left(T=T_{C}\right)$, a dimensão do pacote de onda torna-se da ordem da distância interatômica $\left(\lambda_{d B} \approx d\right)$. Nesse ponto os átomos começam a perder as suas propriedades individuais. (d) Quando temperatura atinge o zero absoluto $(T=0 \mathrm{~K})$, os átomos tornam-se indistinguíveis e comportam-se de maneira coletiva (como se eles sofressem uma "crise de identidade"). Os átomos condensam em um único estado, representado por um pacote de onda gigante; i.e., obtém-se um BEC puro. É interessante notar que essa onda gigante é macroscópica.

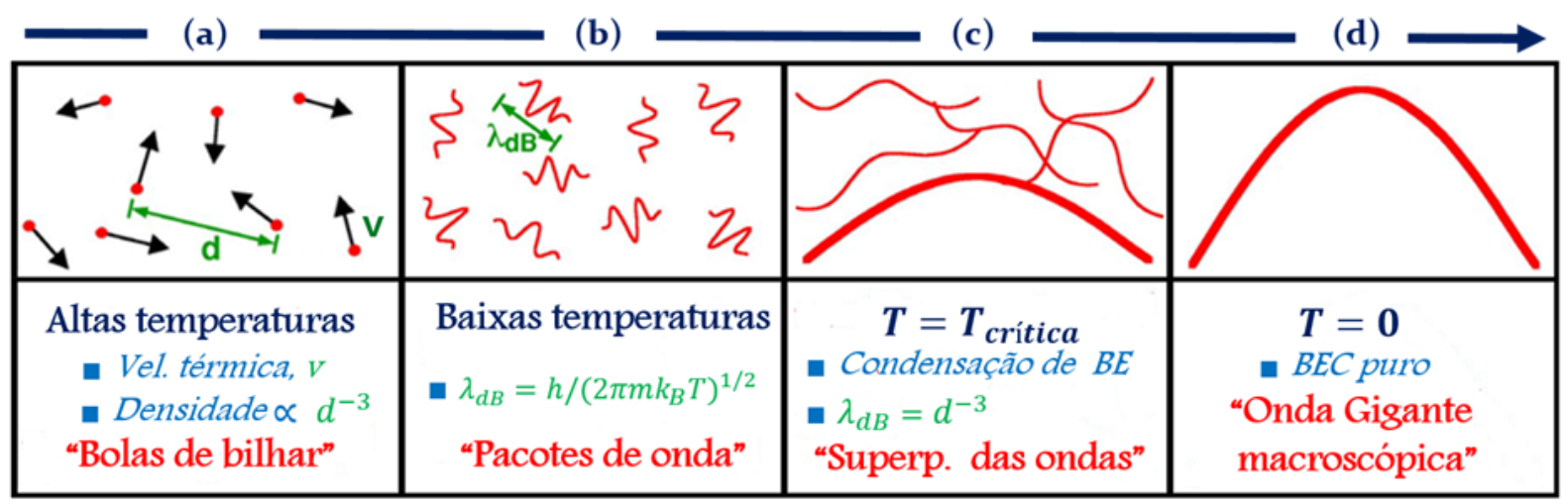

Figura 2 - Etapas que atravessa o gás ideal de bósons diluído até atingir o condensado (BEC). Fonte: Adaptada de ALMEIDA. (39) 


\subsubsection{BEC não interagente: Gás ideal de Bose}

Um sistema de $N$ átomos (bósons) não interagentes e em equilíbrio termodinâmico contidos em um volume $\mathcal{V}$, é descrito através da função de distribuição de Bose-Einstein

$$
\mathscr{F}\left(\varepsilon_{i}, \mu\right)=\left(e^{\left(\varepsilon_{i}-\mu\right) / k_{B} T}-1\right)^{-1}
$$

onde $\mu$ e $k_{B}$ são respectivamente o potencial químico e constante de Boltzmann. $\varepsilon_{i}$ representa a energia de cada estado individual.

Para conservar o número total de átomos do sistema, é comum empregar o formalismo do ensemble grande-canônico: $\Omega=S T-\mu N$, com $N$ definido como

$$
N(T, \mu)=\sum_{i=0}^{\infty} \mathscr{F}\left(\varepsilon_{i}, \mu\right)=\mathscr{F}\left(\varepsilon_{0}, \mu\right)+\sum_{i=1}^{\infty} \mathscr{F}\left(\varepsilon_{i}, \mu=0\right)=N_{0}(T, \mu)+N_{T}(T)
$$

Ao expandir a Eq. (2.3) surgem o número de átomos do condensado, $N_{0}$, e dos estados excitados (térmicos), $N_{T}$; tal que $\varepsilon_{0}$ representa a menor energia dos estados individuais. Aplicando o limite termodinâmico, no espaço de momentos, à equação (2.3), tem-se

$$
N_{T}=\int_{0}^{\infty} \mathscr{F}(\varepsilon) \mathscr{G}(\varepsilon) d \varepsilon \quad \operatorname{com} \quad \mathscr{G}(\varepsilon)=\frac{d \Xi(\varepsilon)}{d \varepsilon}=\mathcal{C} \varepsilon^{\delta-1} .
$$

Onde $\mathscr{G}(\varepsilon)$ representa a densidade de estados, $\Xi(\varepsilon)$ é o número total de estados, $\mathcal{C}$ é uma constante e $\delta(>0)$ um parâmetro que depende do tipo de potencial de confinamento externo.

Ao substituir as equações (2.2) e (2.3) em (2.4), obtém-se

$$
N_{T}=\mathcal{C} \Gamma(\delta) \zeta(\delta)\left(k_{B} T\right)^{\delta}
$$

com $\Gamma(\delta)$ e $\zeta(\delta)$ sendo as funções Gama e Zeta de Riemann.

Por outro lado, o sistema atinge a transição de fase quântica (TFQ) quando o potencial químico é zero $(\mu=0)$, de modo que o número de total de átomos equivale ao número de átomos no estado excitado. Com essa condição surge a temperatura crítica $T_{c}$, que estabelece o limite de temperatura a partir do qual surge o condensado do gás de bósons $\left(T<T_{c}\right)$. Igualando $N=N(T, \mu)=N_{T}\left(T=T_{c}, \mu=0\right)$ na equação $(2.5)$, obtemos

$$
T_{c}=\frac{1}{k_{B}} \sqrt[\delta]{\frac{N}{\mathcal{C} \Gamma(\delta) \zeta(\delta)}}, \quad \longrightarrow \quad \frac{N_{T}}{N}=\left(\frac{T}{T_{c}}\right)^{\delta} .
$$


Combinando as equações (2.6) e (2.3), derivamos o número de átomos presentes no condensado (fração condensada)

$$
\frac{N_{0}}{N}=\left[1-\left(\frac{T}{T_{c}}\right)^{\delta}\right]\left(1-\Theta\left(T-T_{c}\right)\right)
$$

onde $\Theta\left(T-T_{c}\right)$ é a função degrau unitário. Essa relação mostra a dependência entre a temperatura e o número de átomos do condensado. No limite $T \rightarrow 0 \mathrm{~K}$ teremos $N_{0} \sim N$, que por sua vez implica na ocupação macroscópica do estado fundamental $N_{0} \gg N_{T}$.

- Para um potencial de caixa (3D): $\delta=3 / 2$

$$
V=\left\{\begin{array} { l l } 
{ 0 ; \quad x _ { i } \in [ 0 , L ] , \quad x _ { i } = x , y , z } \\
{ \infty ; \quad x _ { i } \geq L \quad \text { e } \quad x _ { i } < 0 , }
\end{array} \text { e } \left\{\begin{array}{ll}
\varepsilon \quad=\mathbf{p}^{2} / 2 m \\
\mathscr{G}(\varepsilon)=\left(\mathcal{V} m^{3 / 2} / \sqrt{2} \pi^{2} \hbar^{3}\right) \varepsilon^{1 / 2}
\end{array}\right.\right.
$$

onde $\mathbf{p}$ representa o momentum do estado atômico individual presente no condensado. Para $T<T_{c}$, de acordo com Eq. (2.7) teremos

$$
\frac{N_{0}}{N}=1-\left(\frac{T}{T_{c}}\right)^{3 / 2}
$$

Empregando $\delta=3 / 2$ na equação (2.5), determina-se a densidade de átomos térmicos

$$
n_{T}=\frac{N_{T}}{\mathcal{V}}=\zeta(3 / 2) \lambda_{d B}^{-3} \quad \Rightarrow \quad n_{T} \lambda_{d B}^{3}=\zeta(3 / 2) \approx 2.612,
$$

onde $\lambda_{d B}$ é o comprimento de onda de de Broglie definido anteriormente. Utilizando a equação (2.8) , computamos o valor da temperatura crítica da transição de fase quântica, na qual temos a saturação da ocupação dos estados excitados e os átomos passam a se acumular no estado fundamental. Esse valor foi obtido por A. Einstein (23) e é conhecido como o critério para formar o condensado.

- Para um potencial de oscilador harmônico (3D): $\delta=3$

$$
V(\mathbf{r})=\frac{m}{2}\left(\omega_{x}^{2} x^{2}+\omega_{y}^{2} y^{2}+\omega_{z}^{2} z^{2}\right)
$$

onde $\left(\omega_{x}, \omega_{y}, \omega_{z},\right)$ são as frequências angulares para cada direção espacial.

$$
\varepsilon=\sum_{i=x}^{z}\left(n_{i}+1 / 2\right) \hbar \omega_{i}, \quad \mathscr{G}(\varepsilon)=\varepsilon^{3} / 2 \hbar^{2} \omega_{x} \omega_{y} \omega_{z},
$$

onde $n_{i} \in \mathbb{Z}$. Para $T<T_{c}$, de acordo com Eq. (2.7) teremos

$$
\frac{N_{0}}{N}=1-\left(\frac{T}{T_{c}}\right)^{3}
$$




\subsubsection{BEC interagente: $\mathrm{T}=\mathrm{OK}$}

A condição (2.1) indica um regime de gás de bósons fracamente interagente e diluído, onde a interação entre os átomos consiste predominantente de colisões binárias. Além disso, como consideramos ultra-baixas temperaturas, podemos adotar espalhamento tipo onda-s, o qual será caracterizado apenas pelo comprimento de espalhamento $a_{s}$.

\section{- Equação de Gross-Pitaevskii}

Segundo a seção 2.1.1, no limite $T \rightarrow 0 \mathrm{~K}$ (BEC puro), os bósons passam a ocupar um mesmo estado quântico no nível de menor energia. Nesse limite em que o número de átomos no condensado é da mesma ordem do número total de átomos $\left(N \approx N_{0}\right)$, o sistema é caracterizado através da função de onda macroscópica, $\Phi(\mathbf{r}, t)$, a qual pode ser obtida pelo produto das funções de onda individuais que caracterizam o átomo no estado fundamental. Para deduzir a GPE, partimos da equação de Heisenberg para dinâmica dos operadores

$$
\begin{aligned}
i \hbar \frac{\partial}{\partial t} \hat{\Psi}(\mathbf{r}, t) & =[\hat{\Psi}(\mathbf{r}, t), \hat{H}] \\
& =\left[-\frac{\hbar^{2} \nabla^{2}}{2 m}+V_{e x t}(\mathbf{r})+\int \hat{\Psi}^{\dagger}\left(\mathbf{r}^{\prime}, t\right) V\left(\mathbf{r}^{\prime}-\mathbf{r}\right) \hat{\Psi}\left(\mathbf{r}^{\prime}, t\right) d \mathbf{r}^{\prime}\right] \hat{\Psi}(\mathbf{r}, t)
\end{aligned}
$$

Com $\hat{H}$ sendo o Hamiltoniano do sistema, definido como

$\hat{H}=\int\left(\frac{-\hbar^{2}}{2 m} \hat{\Psi}^{\dagger}(\mathbf{r}, t) \nabla^{2} \hat{\Psi}(\mathbf{r}, t)+V_{e x t}(\mathbf{r})\right) d \mathbf{r}+\iint \hat{\Psi}^{\dagger}(\mathbf{r}, t) \hat{\Psi}^{\dagger}\left(\mathbf{r}^{\prime}, t\right) V\left(\mathbf{r}^{\prime}-\mathbf{r}\right) \hat{\Psi}(\mathbf{r}, t) \hat{\Psi}\left(\mathbf{r}^{\prime}, t\right) d \mathbf{r}^{\prime} d \mathbf{r}$ onde $\hat{\Psi}^{\dagger}(\hat{\Psi})$ representa operadores de criação (destruição) dos átomos na posição $\mathbf{r}$ e tempo $t, V_{\text {ext }}$ corresponde ao potencial de confinamento externo. $V\left(\mathbf{r}^{\prime}-\mathbf{r}\right)$ representa a interação de contato entre dois átomos e tem a forma (21)

$$
V\left(\mathbf{r}^{\prime}-\mathbf{r}\right)=g_{3 D} \delta\left(\mathbf{r}^{\prime}-\mathbf{r}\right) ; \quad \operatorname{com} \quad g_{3 D}=\frac{4 \pi \hbar^{2} a_{s}}{m},
$$

em termos do comprimento de espalhamento $a_{s}$. O potencial efetivo pode ser repulsivo $\left(g_{3 D}>0\right)$ ou atrativo $\left(g_{3 D}<0\right)$. Essa característica se reflete na formação e estabilidade do BEC. $(40,41)$

Para determinarmos a equação que rege a evolução do parâmetro de ordem (BEC), utilizamos o ansatz de Bogoliubov para o operador de campo $\hat{\Psi}(\mathbf{r}, t)=\Phi(\mathbf{r}, t)+\delta \hat{\Psi}(\mathbf{r}, t)$. O 
termo da flutuação pode ser desprezado perante a aplicação da aproximação de mean-field e o operador de estado passa a ser descrito pela função macroscópica

$$
\hat{\Psi}(\mathbf{r}, t) \approx \Phi(\mathbf{r}, t)
$$

Substituindo as Eqs. (2.11) e (2.12) em (2.10), derivamos a GPE

$$
i \hbar \frac{\partial}{\partial t} \Phi(\mathbf{r}, t)=\left[\frac{(-i \hbar \nabla)^{2}}{2 m}+V_{e x t}(\mathbf{r})+g_{3 D}|\Phi(\mathbf{r}, t)|^{2}\right] \Phi(\mathbf{r}, t) .
$$

Consideramos que a função de onda $\Phi(\mathbf{r}, t)$ da GPE 2.13 evolui no tempo na forma (20)

$$
\Phi(\mathbf{r}, t)=\phi(\mathbf{r}) e^{-i \mu t / \hbar}
$$

Ao substituir a equação (2.14) em (2.13), deduz-se a GPE independentemente do tempo

$$
\left[-\frac{(\hbar \nabla)^{2}}{2 m}+V_{\text {ext }}(\mathbf{r})+g_{3 D}|\phi(\mathbf{r})|^{2}-\mu\right] \phi(\mathbf{r})=0,
$$

com $\phi(\mathbf{r})$ sendo a função de onda dos átomos no estado fundamental. O número total de átomos é dado por $N=\int|\phi(\mathbf{r})|^{2} d \mathbf{r}=\int n(\mathbf{r}) d \mathbf{r}$, onde $n(\mathbf{r})$ é a densidade dos átomos.

As equações (2.13) e (2.15) descrevem convenientemente as propriedades do condensado.

\section{- Regime de Thomas-Fermi (TF)}

Uma solução particular da GPE é obtida assumindo que a interação dos átomos faz com que a variação do perfil de densidade do termo cinético seja mais ameno, com gradientes menores, em comparação com os outros termos. A energia cinética torna-se desprezível fazendo que só os potenciais de interação e de confinamento externo sejam relevantes na dinâmica do condensado. Introduzindo $E_{\text {cine }} \gg E_{\text {pot }}$ na Eq. (2.15), tem-se

$$
\mu \phi(\mathbf{r})=\left(V_{e x t}(\mathbf{r})+g_{3 D}|\phi(\mathbf{r})|^{2}\right) \phi(\mathbf{r}) .
$$

Da solução desta equação temos o perfil de densidade dos átomos:

$$
n(\mathbf{r})=|\phi(\mathbf{r})|^{2}=\left(\frac{\mu-V_{e x t}(\mathbf{r})}{g_{3 D}}\right) \Theta\left[\mu-V_{e x t}(\mathbf{r})\right]
$$

Para obter as dimensões aproximadas da nuvem do condensado é preciso conhecer a forma do potencial externo. Assumindo o potencial de oscilador harmônico da equação (2.9), obtêm-se o comprimento de oscilador $a_{o h}$ e frequência $\omega_{o h}$ desse potencial

$$
a_{o h}=\sqrt{\hbar / m \omega_{o h}}, \quad \omega_{o h}=\sqrt[3]{\omega_{x} \omega_{y} \omega_{z}}
$$


A extensão da nuvem do condensado é determinada aproximadamente com a aplicação da condição de fronteira $V(\mathbf{r})=\mu$, com a qual derivamos o raio de TF para cada eixo

$$
R_{i}^{T F}=\sqrt{\frac{2 \mu}{m \omega_{i}^{2}}}=\sqrt{\frac{2 g_{3 D} n_{0}}{m \omega_{i}}}, \quad i=x, y, z . \quad \underbrace{\Rightarrow}_{(2.17)} \quad \sqrt[3]{R_{x}^{T F} R_{y}^{T F} R_{z}^{T F}}=R^{T F}=a_{o h}^{2} / \xi
$$

onde $n_{0}$ é a densidade central do condensado obtida para $\mathbf{r}=\mathbf{0}$ na equação $(2.16)$ e $\xi$ é o healing length, definido como a escala de comprimento onde o condensado recompõe seu valor uniforme da densidade atômica

$$
\begin{aligned}
& \mu=g_{3 D}|\phi(\mathbf{r}=0)|^{2}=g_{3 D} n_{0}, \\
& \xi=\hbar / \sqrt{2 m g_{3 D} n_{0}} \underbrace{\longrightarrow}_{(2.11)} \xi=1 / \sqrt{8 \pi n_{0} a_{s}}
\end{aligned}
$$

Ao empregar o potencial químico e o healing length no perfil da densidade de átomos, Eq. (2.16), reescrevemos o raio de TF (41) como $R^{T F}=a_{o h}\left(15 N a_{s} / a_{o h}\right)^{1 / 5}$; Essa relação é válida na seguinte escala de comprimento (42): $\xi \ll a_{o h} \ll R^{T F}$.

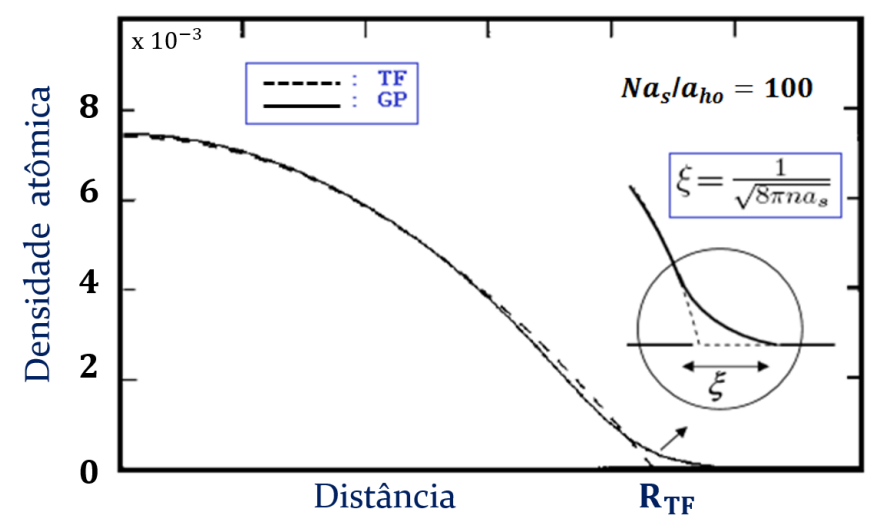

Figura 3 - Perfil de densidade atômica do regime de TF e solução numérica da GPE estacionária, Eq. (2.15). O deslocamento da GPE com respeito ao perfil parabólico de TF é da ordem do healing length. Como $N a_{s} / a_{o h} \sim 100$, o regime de TF é uma boa aproximação para descrever o BEC.

Fonte: Adaptada de DALFOVO et al. (41) 


\subsection{Vórtices no BEC}

Os vórtices são singularidades que surgem como consequência de imprimir uma fase local, que varia no espaço e tempo, na função de onda macroscópica do BEC através de uma excitação externa. O contorno fechado ao redor de um vórtice descreve uma fase total multipla de $2 \pi$, com densidade atômica nessa singularidade sendo nula. Dependendo da excitação externa pode-se cristalizar desde um até um arranjo de vórtices. Diversas técnicas experimentais foram empregadas para excitar as configurações de vórtices em superfluidos de átomos ultrafrios. (3, 15, 43-46)

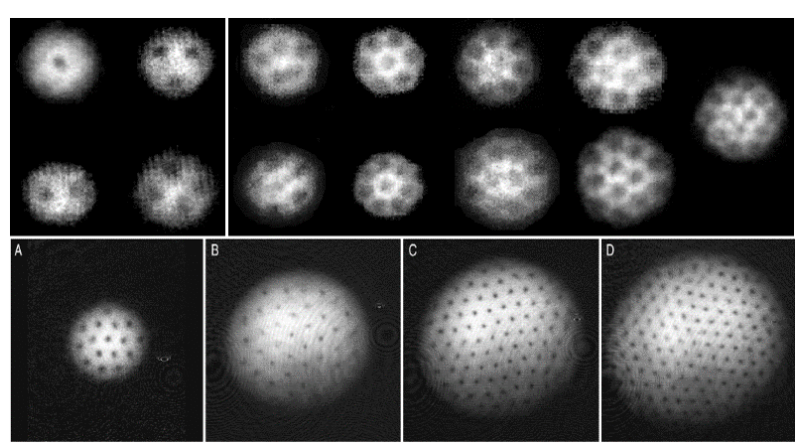

Figura 4 - Imagens experimentais desde 1 até um arranjo de 130 vórtices. Acima: Formação desde 1 até 13 vórtices. O condensado tem seu formato variando de um cigar-shape para um pancake-shape a medida que adquire maior número de vórtices. Abaixo: Cristalização dos vórtices em rede triangular para diferentes tempos na armadilha; observam-se (A) 16, (B) 32, (C) 80 e (D) 130 vórtices.

Fonte: MADISON et al. (43,47), Acima e ABO-SHAEER et al. (15), Abaixo.

- Superfluidez: Quando um fluido é irrotacional, chama-se de superfluido. Empregando a GPE dependente do tempo, Eq. (2.13), tem-se a densidade de corrente: $\mathscr{J}=$ $i(\hbar / 2 m)\left[\Phi(\mathbf{r}, t) \nabla \Phi^{*}(\mathbf{r}, t)-\Phi^{*}(\mathbf{r}, t) \nabla \Phi(\mathbf{r}, t)\right]=|\Phi(\mathbf{r}, t)|^{2} \mathbf{v}(\mathbf{r}, t)$, sendo $\mathbf{v}$ a velocidade local do condensado. A função de onda do BEC pode ser escrita usando a relação de Madelung

$$
\Phi(\mathbf{r}, t)=\sqrt{n(\mathbf{r}, t)} e^{i \theta(\mathbf{r}, t)}
$$

onde $\theta(\mathbf{r}, t)$ representa a fase local do condensado.

Ao substituir a Eq. (2.21) na densidade de corrente, obtêm-se a velocidade

$$
\mathbf{v}(\mathbf{r}, t)=(\hbar / m) \nabla \theta(\mathbf{r}, t) \quad \text { cujo rotacional } \quad \nabla \times \mathbf{v}(\mathbf{r}, t)=0 .
$$


A irrotacionalidade indica que o BEC é um superfluido.

\subsubsection{Vórtice unitário: Quantização da circulação}

Considerando a velocidade do superfluido descrevendo um contorno fechado na vizinhança de um vórtice (Figura 5), é possível determinar a circulação $\mathscr{C}$. (48) Usando equação (2.22), consegue-se

$$
\mathscr{C}=\oint_{C \in[0,2 \pi]} \mathbf{v} \cdot d \mathbf{r}=\frac{\hbar}{m} \oint_{C \in[0,2 \pi]} \nabla \theta \cdot d \mathbf{r}=\frac{\hbar}{m} \oint_{C \in[0,2 \pi]} d \theta \Rightarrow \mathscr{C}=2 \pi \frac{\hbar}{m} q ; \quad q \in \mathbb{Z}
$$

Essa relação é conhecida como a condição de quantização de Onsager-Feynman. O parâmetro $q$ pode ser entendido como a carga do vórtice ( $q=1$ corresponde a carga unitária).

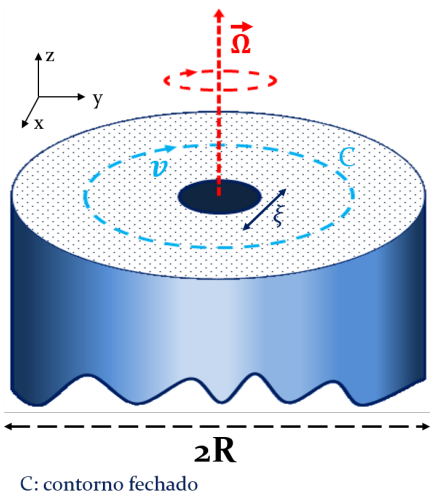

Figura 5 - Superfluído aprisionado em armadilha cilíndrica com frequência angular $\Omega=\Omega \hat{z}$. Acima de um valor crítico de $\Omega$ temos o aparecimento das excitações de vórtice no superfluido. $R$ e $v$ são o raio do cilindro e velocidade do superfluido.

Fonte: Elaborada pelo autor. 


\subsubsection{BEC em rotação: Rede de Vórtices}

O Hamiltoniano do sistema no referencial em rotação pode ser descrito usando a definição de Landau (49) como

$$
\begin{aligned}
& H_{\text {rot }}=H_{l a b}-\boldsymbol{\Omega} \cdot \mathbf{L}, \\
& H_{l a b}=\frac{\mathbf{p}^{2}}{2 m}+V_{o h},
\end{aligned}
$$

onde $\mathbf{L}=\mathbf{r} \times \mathbf{p}$ é o momento angular total e $H_{l a b}$ é o Hamiltoniano que descreve os átomos do superfluido no referencial em repouso, com confinamento harmônico dado por

$$
V_{\text {oh }}=\frac{m}{2}\left[\omega_{\rho}^{2}\left(x^{2}+y^{2}\right)+\omega_{z}^{2} z^{2}\right] ; \quad r^{2}=\rho^{2}+z^{2},
$$

onde $\omega_{\rho}$ é a frequência angular radial e $\omega_{z}$ frequência angular axial paralela ao eixo de rotação. Usando a equação (2.24) em (2.23), deriva-se

$$
H_{\text {rot }}=\frac{(\mathbf{p}-\mathbf{A})^{2}}{2 m}+\frac{m}{2} \omega_{e f}^{2} \rho^{2}+\frac{m}{2} \omega_{z}^{2} z^{2}, \quad \text { com } \quad \mathbf{A}=m \boldsymbol{\Omega} \times \mathbf{r} \quad \text { e } \quad \omega_{e f}=\sqrt{\omega_{\rho}^{2}-\Omega^{2}} .
$$

O campo vetorial A pode visto como um análogo do potencial vetorial magnético (ou potencial de calibre) induzido pelas forças de Lorentz atuando em um campo magnético uniforme. (50) A frequência efetiva $\omega_{\text {ef }}$ permite estabelecer o regime adequado de estudo dos vórtices, que depende da relação entre $\omega_{\rho}$ e $\Omega$. (42) Definimos o fator

$$
\bar{\Omega}=\Omega / \omega_{\rho}
$$

Ressaltando que para $\bar{\Omega} \rightarrow 1$ o condensado tende a formar um disco (o condensado estendese radialmente), e em um caso extremo pode ser tratado como um sistema quase-2D. Por outro lado, o termo cinético da Eq. (2.26) tende à zero quando $\mathbf{p}=\mathbf{A}$, de onde obtém-se a velocidade de corpo rigido, tal como acontece para um fluido clássico (21)

$$
\mathbf{v}=\Omega \times \mathbf{r}, \quad \longrightarrow \quad \nabla \times \mathbf{v}=2 \Omega(\neq 0)
$$

O condensado excitado com frequência angular constante $\Omega$ apresenta vorticidade, dado pela Eq. (2.28), aproximadamente uniforme. Esse resultado traz uma nova escala de comprimento com o aparecimento da rede de vórtices. Seguindo o critério de Fetter (42), ver 
Figura 6b, os vórtices podem ser cinscunscritos em celas circulares; o raio a dessa cela é demoninado de magnetic length devido à analogia feita com o campo magnético do vetor A. Partindo da circulação para uma rede (51), seja

$$
\mathscr{C}=N_{v} \frac{2 \pi \hbar}{m}=\oint_{C(\mathcal{S})} \mathbf{v} \cdot d \mathbf{l}=\oint_{\mathcal{S}}(\nabla \times \mathbf{v}) \cdot d \mathbf{s}=2 \Omega \mathcal{S}, \quad \text { com } \quad n_{v}=\frac{m \Omega}{\pi \hbar},
$$

onde $N_{v}=n_{v} \mathcal{S}$ é o número de vórtices e $\mathcal{S}=\pi a^{2}$. Para $N_{v}=1$ e usando as Eqs. (2.28)-(2.29)

$$
\mathscr{C}=2 \Omega \mathcal{S}=\frac{2 \pi \hbar}{m} \longrightarrow a=\sqrt{\frac{\hbar}{m \Omega}} .
$$

O aumento gradativo da ação da excitação externa provoca um aumento no número de vórtices $N_{v}$ da nuvem condensada, até o ponto em que eles se re-arranjam para estabelecer uma rede triangular estável (15), ver Figura 6a. A estabilidade da rede de vórtice pode ser estabelecida com base no fator de preenchimento

$$
\nu=N / N_{v}
$$

Para $\nu>\nu_{c}$ o condensado em rotação atinge estabilidade formando uma rede triangular de vórtices, enquanto que é instavel para $\nu<\nu_{c}$, apresentando fases fortemente correlacionadas a partir de um certo valor crítico $\nu_{c} \sim 1$. (50)

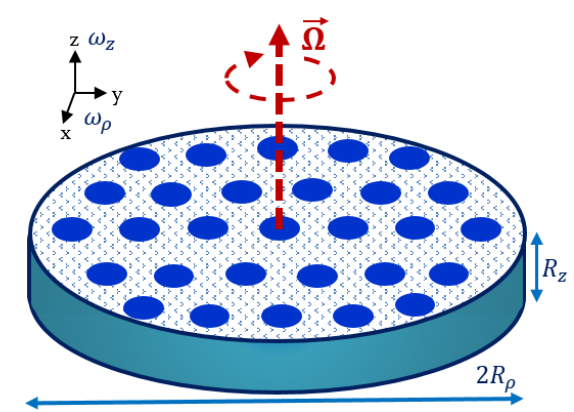

(a)

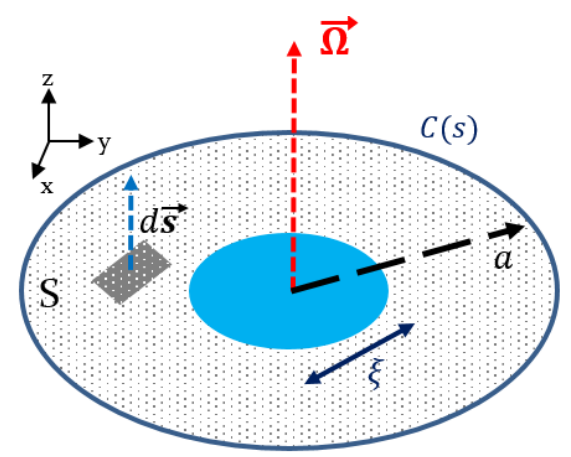

(b)

Figura 6 - (a) BEC quase $2 D$ composto de um arranjo de vórtices. (b) Vórtice unitário rodeado pelo magnetic length a. O núcleo do vórtice é da ordem do healing length, $\xi$.

Fonte: Elaborada pelo autor.

Os raios de TF (componentes radial e azimutal) para um sistema em rotação são redefinidos de acordo com as equações (2.18) e (2.26), adotando a forma

$$
R_{\rho}=\sqrt{\frac{2 g_{3 D} n_{0}}{m \omega_{e f}^{2}}} \text { e } \quad R_{z}=\sqrt{\frac{2 g_{3 D} n_{0}}{m \omega_{z}^{2}}} .
$$




\section{3 Átomos ultrafrios em redes ópticas}

Neste capítulo apresentam-se as características físicas e descrição matemática das redes ópticas. (52) Na sequência mostram-se tanto a proposta teórica como a verificação experimental do comportamento dos átomos ultrafrios (BEC) na presença da rede óptica.

\subsection{Redes ópticas}

Uma rede óptica é um arranjo periódico de milhares de micro potenciais ópticos advindos da interferência dos feixes de lasers. $(53,54)$ Os potenciais periódicos são gerados pela interferência de dois feixes de lasers propagando-se em direções opostas, os quais geram um campo elétrico oscilante no espaço (isto é, são ondas estacionárias que apresentam um perfil de intensidade espacial). O életron no átomo na presença desse campo atinge um momento dipolar dependente do tempo. Por causa do dipolo e o campo elétrico, a energia eletromagnética experimenta um deslocamento quadrático do campo elétrico (efeito Stark). Então, os átomos são empurrados para posições de mínima ou máxima intensidade dependendo se a frequência do laser é muito menor ou muito maior do que a frequência da transição atômica. (54)

Consideramos a sobreposição de dois feixes de lasers contra-progantes ao longo da direção $\mathrm{x}$ :

$$
\mathbf{E}(x, t)=\mathbf{E}_{0} \cos \left(k_{l} x-\omega t\right)+\mathbf{E}_{0}^{*} \cos \left(k_{l} x+\omega t\right)
$$

onde $\omega$ é a frequência, $k_{l}$ o vetor de onda e $\mathbf{E}(x, t)$ é o campo elétrico dos lasers.

Ao determinar a média com respeito ao tempo do módulo quadrado do campo elétrico, obtém-se o potencial óptico (10)

$$
V(x) \sim\left\langle\left[|\mathbf{E}(x, t)|^{2}\right]_{t}\right\rangle \rightarrow V(x) \simeq \frac{\left|\Omega_{0}(x)\right|^{2}}{4 \delta^{2}}=\frac{\Omega_{0}^{2}}{4 \delta^{2}} \cos ^{2}\left(k_{l} x\right), \rightarrow V(x) \simeq V_{0} \cos ^{2}\left(k_{l} x\right)
$$


onde $\Omega_{0}$ é a frequência de Rabi e $\delta$ é o detuning (diferença entre as frequências atômica e da radiação), enquanto que $V_{0}$ caracteriza a profundidade do potencial periódico. Definimos a energia de recuo $E_{R}$ como a escala de energia natural dos átomos em redes ópticas (13)

$$
E_{R}=\frac{\hbar^{2} k_{l}^{2}}{2 m}
$$

com $m$ sendo a massa do átomo.

O potencial periódico formado traz uma periodicidade definida por $d=\lambda / 2$, sendo $\lambda=2 \pi / k_{l}$ o comprimento de onda dos feixes de lasers. Existem muitas propostas para geração das redes estacionárias; a equação com os feixes contrapropagantes (3.1) pode ser estendida para fornecer as redes quadradas em duas ou três dimensões espaciais, ou até mesmo redes triangulares ou hexagonais $(55,56)$, com a utilização de diferentes números de lasers nos ângulos apropriados.
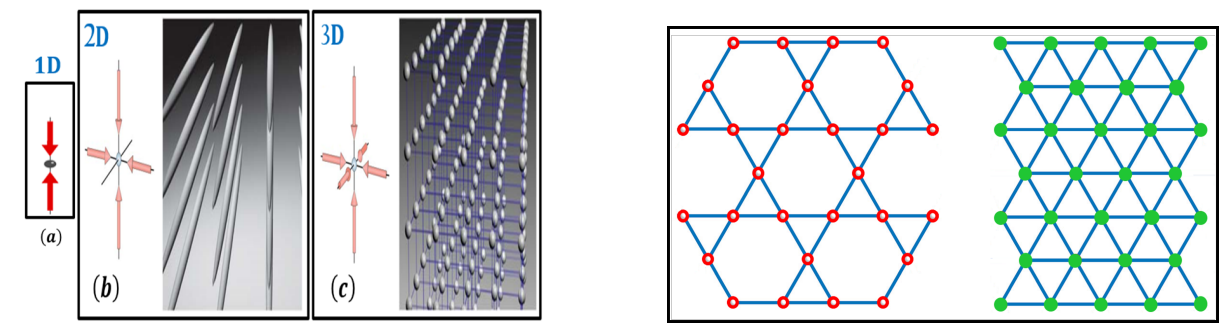

Figura 7 - Diferentes configurações espaciais da rede óptica. Esquerda: (a) 1D, (b) 2D, (c) $3 D$. Direita: Configurações geradas quando se mudam as direções dos lasers em $2 D$ tais como hexagonal e triangular.

Fonte: BLOCH (53), à esquerda e Elaborada pelo autor, à direita.
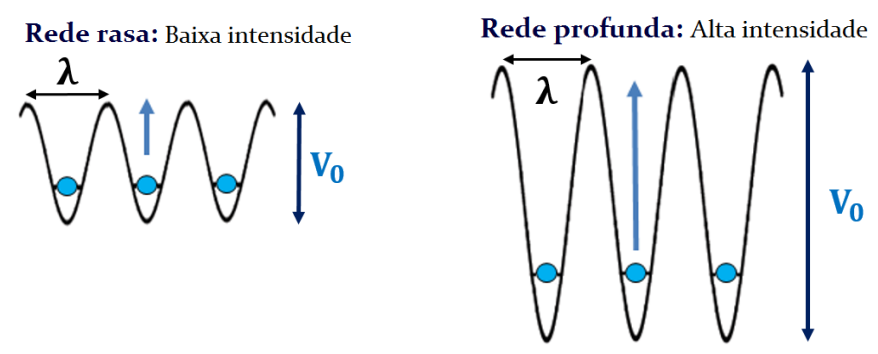

Figura 8 - Redes rasa e profunda obtidas variando externamente a intensidade dos feixes de lasers.

Fonte: Elaborada pelo autor. 
Conforme ilustrado, o controle da intensidade, comprimentos de onda e direção dos feixes de lasers permitem manipular e criar experimentalmente redes arbitrarias, em qualquer dimensão espacial. Vale ressaltar que, diferentemente das redes do estado sólido, as redes ópticas são redes estáticas (ausência de modos vibracionais - fônons) e livres de defeitos. Essas suas características é que permitiram a obtenção da transição de fase quântica em átomos fortemente correlacionados. (57)

\subsection{Bandas de energia}

Tanto a rede óptica como os sólidos estudados na física de matéria condensada são compostos de potencias periódicos. Essa característica sugere empregar o formalismo matemático do estado sólido às redes ópticas, i.e., pode-se fazer uma equivalência entre a rede óptica e a rede de Bravais. O potencial óptico corresponderia à um potencial externo para os átomos, os quais teriam Hamiltoniano dado por

$$
H=-\frac{\hbar^{2} \nabla^{2}}{2 m}+V(\mathbf{r})
$$

onde $V(\mathbf{r})=V(\mathbf{r}+\mathbf{R})$, com $\mathbf{R}$ pertencente à rede óptica, satisfaz a invariâcia translacional. A função de onda do átomo na rede satisfaz a equacao de Schrödinger

$$
H \varphi_{n, \mathbf{k}}(\mathbf{r})=\epsilon_{n} \varphi_{n, \mathbf{k}}(\mathbf{r})
$$

Usando o teorema de Bloch (58) obtém-se as autofunções do Hamiltoniano, Eq. (3.3)

$$
\varphi_{n, \mathbf{k}}(\mathbf{r})=e^{i \mathbf{k} \cdot \mathbf{r}} u_{n, \mathbf{k}}(\mathbf{r})
$$

onde $\mathbf{k}$ é o momentum linear, $u_{n, \mathbf{k}}(\mathbf{r})$ representa uma função com periodicidade da rede $u_{n, \mathbf{k}}(\mathbf{r})=u_{n, \mathbf{k}}(\mathbf{r}+\mathbf{R})$, e $n$ indica a banda de energia.

As autofunções, equação (3.5), podem ser reescritas em termos da periodicidade da rede como

$$
\varphi_{n, \mathbf{k}}(\mathbf{r}+\mathbf{R})=e^{i \mathbf{k} \cdot \mathbf{R}} \varphi_{n, \mathbf{k}}(\mathbf{r})
$$


Expandindo em um conjunto completo de ondas planas tanto a função de Bloch na Eq. (3.5), como o potencial $V(\mathbf{r})$ nas formas $\varphi_{n, \mathbf{k}}(\mathbf{r})=\sum_{\mathbf{k}} c_{n, \mathbf{k}} e^{i \mathbf{k} \cdot \mathbf{r}}$ e $V(\mathbf{r})=\sum_{\mathbf{k}} V_{\mathbf{k}} e^{i \mathbf{k} \cdot \mathbf{r}}$, e substituindo na equação (3.4), obtém-se a equação de Schrödinger no espaço de momentos (59)

$$
\left(\frac{\hbar^{2} \mathbf{k}^{2}}{2 m}-\epsilon\right) c_{n, \mathbf{k}}+\sum_{\mathbf{k}} V_{\mathbf{k}} c_{n, \mathbf{k}-\mathbf{K}}=0
$$

onde $\mathbf{k}$ e $\mathbf{K}$ pertencem à rede recíproca.

A primeira zona de Brillouin (1ZB) representa a recíproca da cela de Wigner-Seitz (WS). Enquanto que a cela WS é definida no espaço real, a $1 Z B$ está descrita no espaço de momentos.

As bandas de energia são obtidas resolvendo a equação matricial (3.7) tanto de forma analítica como numérica no espaço recíproco. $(58,59)$ A Figura 9, mostram a evolução das bandas de energia com relação à profundidade da rede óptica. (a) Bandas de energia típicas de um átomo livre, $\epsilon_{\mathbf{k}}=\hbar^{2} \mathbf{k}^{2} / 2 m$. (b) Quando o potencial é raso, formamse os chamados gaps de energia entre as bandas, mas ainda assim o espectro de energia é comparável ao caso de um átomo livre; as funções de Bloch podem ser aproximadas como ondas planas $\varphi_{\mathbf{k}}(\mathbf{r}) \sim e^{i \mathbf{k} \cdot \mathbf{r}}$ na menor banda de energia. (c) Aqui o potencial é muito profundo e o gap entre as bandas de energia é muito grande. O tamanho do gap pode ser relacionado com a energia de confinamento do átomo nos sítios da rede como $\epsilon_{g a p} \sim \hbar \omega_{l}$, onde a frequência $\omega_{l}$ é obtida expandindo a Eq. (3.1): $V(x) \approx V_{0} k_{l}^{2} x^{2} \approx\left(m \omega_{l}^{2} / 2\right) x^{2}$. Usando o valor de $k_{l}=\pi / d$ e a Eq. (3.2), obtém-se $\omega_{l}=\sqrt{4 V_{0} E_{R}} / \hbar$. Para descrever apropriadamente uma rede profunda, é usual empregar o limite de tight-binding.

\subsubsection{Rede profunda: Aproximação de tight-binding}

O regime de tight-binding é atingido quando as larguras das bandas são muito menores que o gap de energia entre elas (60), comforme mostrado na Figura 9(c). Esse regime resulta apropriado quando os átomos confinados na rede óptica se localizam individual- 

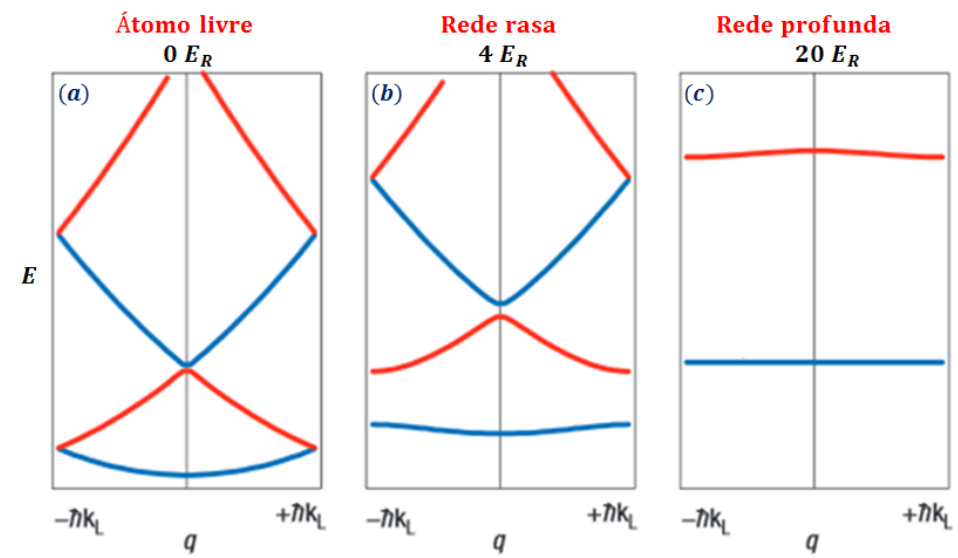

Figura 9 - Bandas de energia (circunscritos no esquema da zona reduzida) de um átomo com a profundidade do potencial óptico $V_{0}$ dada em termos da energia de recuo $E_{R}$. (a) $V_{0}=0$ átomo livre. (b) $V_{0}=4 E_{R}$ rede rasa. (c) $V_{0}=20 E_{R}$ rede profunfa.

Fonte: BLOCH. (53)

mente nos sítios, existindo pequena sobreposição entre suas funções de onda. As funções de onda de Bloch podem ser expandidas em termos de uma função (base) localizada, definida nos sítios da rede

$$
\varphi_{n, \mathbf{k}}(\mathbf{r})=\frac{1}{\sqrt{N_{s}}} \sum_{i} e^{i \mathbf{k} \cdot \mathbf{r}} \phi_{n}\left(\mathbf{r}-\mathbf{R}_{i}\right)
$$

onde $N_{s}$ é o número total de sítios da rede e $\mathbf{R}_{i}$ é o vetor posição de cada sítio. O índice $n$ de $\phi_{n}\left(\mathbf{r}-\mathbf{R}_{i}\right)$ corresponde ao índice de banda. Um caso especial desse tipo de funções é a chamada função de Wannier. (61)

As funções de Bloch satisfazem a condição de ortogonalidade

$$
\int \varphi_{n^{\prime}, \mathbf{k}^{\prime}}^{*}(\mathbf{r}) \varphi_{n, \mathbf{k}}(\mathbf{r}) d \mathbf{r}=\delta_{\mathbf{k}, \mathbf{k}^{\prime}} \delta_{n, n^{\prime}}
$$

\subsubsection{Funções de Wannier}

Para descrever os átomos da rede óptica, no limite de tight-binding usa-se a função de Wannier na menor banda energética $(n=0)$. Em forma geral, a função de Wannier é 
dada pela combinação linear das funções de Bloch (62)

$$
w_{n}\left(\mathbf{r}-\mathbf{R}_{i}\right)=\frac{1}{\sqrt{N_{s}}} \sum_{\mathbf{k}} e^{-i \mathbf{k} \cdot \mathbf{R}_{i}} \varphi_{n, \mathbf{k}}(\mathbf{r})
$$

Fazendo

$$
\begin{aligned}
& \frac{1}{\sqrt{N_{s}}} \sum_{i} e^{i \mathbf{k}^{\prime} \cdot \mathbf{R}_{i}} w_{n}\left(\mathbf{r}-\mathbf{R}_{i}\right)=\sum_{\mathbf{k}}\left[\frac{1}{N_{s}} \sum_{i} e^{i\left(\mathbf{k}^{\prime}-\mathbf{k}\right) \cdot \mathbf{R}_{i}}\right] \varphi_{n, \mathbf{k}}(\mathbf{r}) \\
&=\sum_{\mathbf{k}} \delta_{\mathbf{k}, \mathbf{k}^{\prime}} \varphi_{n, \mathbf{k}}(\mathbf{r})=\varphi_{n, \mathbf{k}^{\prime}}(\mathbf{r}) \\
& \varphi_{n, \mathbf{k}}(\mathbf{r})=\frac{1}{\sqrt{N_{s}}} \sum_{i} e^{i \mathbf{k} \cdot \mathbf{R}_{i}} w_{n}\left(\mathbf{r}-\mathbf{R}_{i}\right)
\end{aligned}
$$

Se $w_{0}\left(\mathbf{r}-\mathbf{R}_{i}\right) \equiv w\left(\mathbf{r}-\mathbf{R}_{i}\right)$ e $\varphi_{0, \mathbf{k}}(\mathbf{r}) \equiv \varphi_{\mathbf{k}}(\mathbf{r})$, consegue-se

$$
w\left(\mathbf{r}-\mathbf{R}_{i}\right)=\frac{1}{\sqrt{N_{s}}} \sum_{\mathbf{k}} e^{-i \mathbf{k} \cdot \mathbf{R}_{i}} \varphi_{\mathbf{k}}(\mathbf{r})
$$

Empregando as Eqs. (3.9) e (3.12), deriva-se a condição de ortogonalidade

$$
\begin{aligned}
\int w^{*}\left(\mathbf{r}-\mathbf{R}_{i}\right) w^{*}\left(\mathbf{r}-\mathbf{R}_{j}\right) d \mathbf{r} & =\frac{1}{N s} \sum_{\mathbf{k}, \mathbf{k}^{\prime}} e^{i \mathbf{k}^{\prime} \cdot \mathbf{R}_{i}-i \mathbf{k} \cdot \mathbf{R}_{j}}\left[\int \varphi_{\mathbf{k}^{\prime}}^{*}(\mathbf{r}) \varphi_{\mathbf{k}}(\mathbf{r}) d \mathbf{r}\right]=\frac{1}{N_{s}} \sum_{\mathbf{k}} e^{i \mathbf{k} \cdot\left(\mathbf{R}_{i}-\mathbf{R}_{j}\right)} \\
& =\delta_{\mathbf{R}_{i}, \mathbf{R}_{j}}=\delta_{i, j}
\end{aligned}
$$

\section{3 $B E C$ em redes ópticas}

Um dos grandes progressos no estudo dos átomos frios foi observar a TFQ em condensados de Bose-Einstein imersos em uma rede óptica. O BEC é um sistema de átomos fracamente interagentes que é induzido adiabaticamente a ter correlações interatômicas fortes (altamente interagente). Essa mudança se traduz em novas fases do sistema, que deverão ser estudadas através de novos formalismos matemáticos. No sistema estudado aqui empregaremos o modelo de Bose-Hubbard, o qual será visto no capítulo seguinte . 


\subsubsection{Observação experimental}

No ano 2002, Greiner (63) verificou experimentalmente a TFQ predita por Jaksch et al. (13), utilizando átomos de ${ }^{87} \mathrm{Rb}$ confinados em uma rede óptica tridimensional.

A Figura 10 mostra os padrões de interferência da amostra de átomos ultrafrios para diferentes valores de profundidade do potencial $V_{0}$. As imagens se referem ao espaço de momentos, e foram construídas por meio da técnica de imagem por absorção. Para redes rasas $(a)-(d)$, os átomos apresentam uma grande coerência, i.e. todos eles estão "sintonizados" e delocalizados na rede toda comportando-se como uma onda gigante (comportamento típico do BEC). Esse estado é chamado de fase superfluida. Quando a profundidade da rede se aproxima do valor $V_{0}=13 E_{R}(e)$, o sistema atinge o ponto crítico da TFQ. A partir desse ponto os átomos começam a perder sua coerência e tornam-se "independentes" ocupando cada sítio possível da rede, i.e., eles alcançam a fase chamada de isolante de Mott.

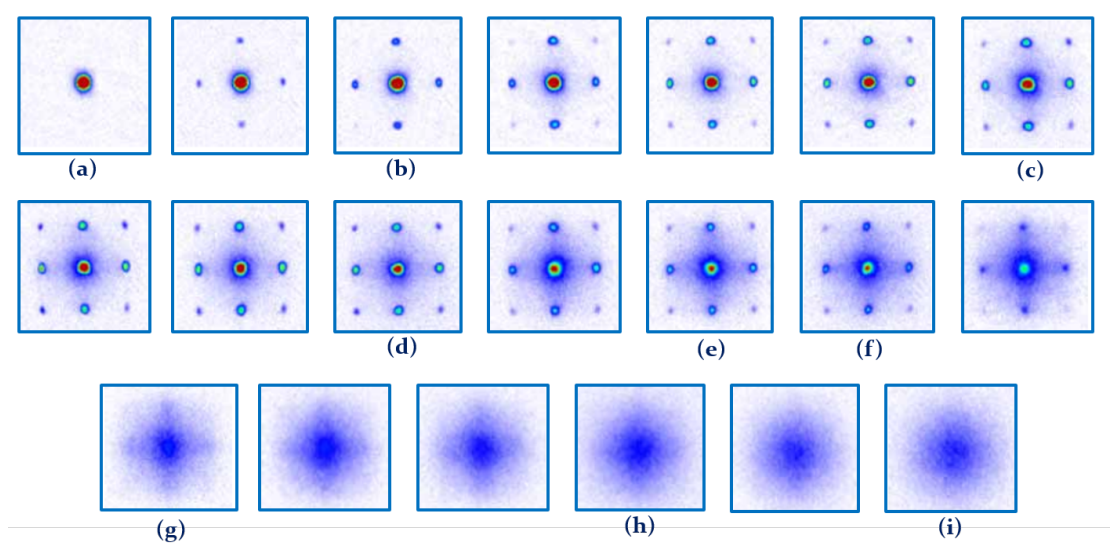

Figura 10 - Etapas atravessadas pela amostra aprisionada até atingir a TFQ. As imagens se referem à distribuicao dos átomos no espaço dos momentos. Os valores de $V_{0}=$ $(a): 0 E_{R},(b): 3 E_{R},(c): 7 E_{R},(d): 10 E_{R},(e): 13 E_{R},(f): 14 E_{R},(g): 16 E_{R}$, $(h): 20 E_{R}$ e $(i): 22 E_{R}$.

Fonte: GREINER et al. $(63,64)$ 



\section{Modelo de Bose-Hubbard}

No ano 1963, J. Hubbard (65) propõe um método aproximado para descrever o comportamento dos elétrons em um sólido (elétrons interagindo em uma rede de potenciais periódicos). Nesse modelo, o balanço entre as energias cinética e de interação fazem com que os elétrons passem de correlações fracas para fortes, induzindo novas fases no sistema.

Por outro lado, é interessante notar que o modelo de Hubbard para baixas temperaturas $(T \approx 0 \mathrm{~K})$ é uma boa aproximação para descrever átomos (férmions ou bósons) confinados em potenciais periódicos. O modelo de Hubbard dos elétrons foi aplicado a sistemas de bósons por M. Fisher (12) em 1989, para descrever a destruição da superfluidez sob efeitos das interações fortes. Quando o objeto de estudo são bósons, denomina-se modelo de Bose-Hubbard (BHM).

\subsection{Hamiltoniano de Bose-Hubbard}

A prescrição empregada para estudar um gás ultrafrio composto de $N$ átomos é o critério da segunda quantização, onde as funções de onda tornam-se autovetores de estado, cujo autovalor é controlado com a atuação dos operadores de criação e destruição.

Seja o Hamiltoniano para um sistema de $N$ átomos fracamente interagente na linguagem da segunda quantização

$$
\hat{H}=\int \hat{\psi}^{\dagger}(\mathbf{r})\left[-\frac{\hbar^{2} \nabla^{2}}{2 m}+V_{e x t}(\mathbf{r})-\mu_{0}\right] \hat{\psi}(\mathbf{r}) d \mathbf{r}+\frac{1}{2} \iint \hat{\psi}^{\dagger}\left(\mathbf{r}^{\prime}\right) \hat{\psi}^{\dagger}(\mathbf{r}) U\left(\mathbf{r}^{\prime}-\mathbf{r}\right) \hat{\psi}\left(\mathbf{r}^{\prime}\right) \hat{\psi}(\mathbf{r}) d \mathbf{r} d \mathbf{r}^{\prime}
$$

onde $U\left(\mathbf{r}^{\prime}-\mathbf{r}\right)$ representa o potencial de interação de dois corpos, $V_{\text {ext }}(\mathbf{r})$ é o potencial externo periódico e $\mu_{0}$ o potencial químico, que garante a conservação dos átomos.

Os operadores de estado $\hat{\psi}\left(\hat{\psi}^{\dagger}\right)$ destroem (criam) átomos na posição r, e satisfazem as 
relações de comutação (20)

$$
\left[\hat{\psi}(\mathbf{r}), \hat{\psi}^{\dagger}\left(\mathbf{r}^{\prime}\right)\right]=\delta\left(\mathbf{r}-\mathbf{r}^{\prime}\right), \quad\left[\hat{\psi}^{\dagger}(\mathbf{r}), \hat{\psi}^{\dagger}\left(\mathbf{r}^{\prime}\right)\right]=\left[\hat{\psi}(\mathbf{r}), \hat{\psi}\left(\mathbf{r}^{\prime}\right)\right]=0
$$

Para regimes de baixa temperatura e densidade (como o caso do BEC), o potencial de interação se reduz a uma interação de contato

$$
U\left(\mathbf{r}^{\prime}-\mathbf{r}\right)=g_{3 D} \delta\left(\mathbf{r}-\mathbf{r}^{\prime}\right)
$$

onde $m$ e $a_{s}$ são a massa do átomo e o comprimento de espalhamento da onda-s.

Substituindo a equação (4.2) em (4.1), obtém-se o Hamiltoniano

$$
\hat{H}=\int \hat{\psi}^{\dagger}(\mathbf{r})\left[-\frac{\hbar^{2} \nabla^{2}}{2 m}+V_{e x t}(\mathbf{r})-\mu_{0}\right] \hat{\psi}(\mathbf{r}) d \mathbf{r}+\frac{g_{3 D}}{2} \int \hat{\psi}^{\dagger}(\mathbf{r}) \hat{\psi}^{\dagger}(\mathbf{r}) \hat{\psi}(\mathbf{r}) \hat{\psi}(\mathbf{r}) d \mathbf{r}
$$

Os átomos em redes profundas podem ser descritos com a aproximação de tightbinding. Usando esse critério os operadores de estado são expandidos em termos das funções de Wannier localizadas nos sítios da rede $R_{i}$ (ver seção 3.2.2)

$$
\hat{\psi}(\mathbf{r})=\sum_{i} w\left(\mathbf{r}-\mathbf{R}_{i}\right) \hat{a}_{i} \quad \text { e } \quad \hat{\psi}^{\dagger}(\mathbf{r})=\sum_{i} w^{*}\left(\mathbf{r}-\mathbf{R}_{i}\right) \hat{a}_{i}^{\dagger} .
$$

Em Eq. (4.4), w(r $\left.-\mathbf{R}_{i}\right)$ representa a função de Wannier para átomos na menor banda de energia de Bloch. Os operadores $\hat{a}_{i}$ e $\hat{a}_{i}^{\dagger}$ são os operadores de criação e destruição dos átomos nos sítios, e satisfazem as relações de comutação:

$$
\left[\hat{a}_{i}, \hat{a}_{j}^{\dagger}\right]=\delta_{i, j}, \quad\left[\hat{a}_{i}^{\dagger}, \hat{a}_{j}^{\dagger}\right]=\left[\hat{a}_{i}, \hat{a}_{j}\right]=0
$$

O operador número é definido como

$$
\hat{n}_{i}=\hat{a}_{i}^{\dagger} \hat{a}_{i}
$$

Ao substituir a equação (4.4) em (4.3), tem-se

$$
\begin{aligned}
\hat{H}= & \sum_{i j}\left\{\int w^{*}\left(\mathbf{r}-\mathbf{R}_{i}\right)\left[-\frac{\hbar^{2} \nabla^{2}}{2 m}+V_{e x t}(\mathbf{r})\right] w\left(\mathbf{r}-\mathbf{R}_{j}\right) d \mathbf{r}\right\} \hat{a}_{i}^{\dagger} \hat{a}_{j} \\
& -\mu_{0} \sum_{i j}\left\{\int w^{*}\left(\mathbf{r}-\mathbf{R}_{i}\right) w\left(\mathbf{r}-\mathbf{R}_{j}\right) d \mathbf{r}\right\} \hat{a}_{i}^{\dagger} \hat{a}_{j} \\
& +\frac{g_{3 D}}{2} \sum_{i j k l}\left[\int w^{*}\left(\mathbf{r}-\mathbf{R}_{i}\right) w^{*}\left(\mathbf{r}-\mathbf{R}_{k}\right) w\left(\mathbf{r}-\mathbf{R}_{j}\right) w\left(\mathbf{r}-\mathbf{R}_{l}\right) d \mathbf{r}\right] \hat{a}_{i}^{\dagger} \hat{a}_{k}^{\dagger} \hat{a}_{j} \hat{a}_{l} \\
= & -\sum_{i j} J_{i j} \hat{a}_{i}^{\dagger} \hat{a}_{j}-\mu_{0} \sum_{i j} \hat{a}_{i}^{\dagger} \hat{a}_{j}+\frac{1}{2} \sum_{i j k l} U_{i j k l} \hat{a}_{i}^{\dagger} \hat{a}_{k}^{\dagger} \hat{a}_{j} \hat{a}_{l} .
\end{aligned}
$$


O Hamiltoniano de Bose-Hubbard (BH) (11) advém da Eq. (4.6) com a consideração das funções de Wannier localizadas, que permite restrigirmos o tunelamento entre os primeiros vizinhos da rede $\left(J_{i j}\right)$, e com a energia de interação $\left(U_{i j k l}\right)$ restritas ao mesmo sítio. Assim

$$
\hat{H}_{B H}=-J \sum_{\langle i j\rangle} \hat{a}_{i}^{\dagger} \hat{a}_{j}-\mu \sum_{i} \hat{n}_{i}+\frac{U}{2} \sum_{i} \hat{n}_{i}\left(\hat{n}_{i}-1\right) .
$$

Aqui $\langle i j\rangle$ denota o somatório sobre todos os pares de primeiros vizinhos, e

$$
\begin{aligned}
& J=-\int w^{*}\left(\mathbf{r}-\mathbf{R}_{i}\right)\left[\frac{-\hbar^{2} \nabla^{2}}{2 m}+V_{e x t}(\mathbf{r})\right] w\left(\mathbf{r}-\mathbf{R}_{j}\right) d \mathbf{r}, \\
& \mu=\mu_{0}-\int w^{*}\left(\mathbf{r}-\mathbf{R}_{i}\right)\left[\frac{-\hbar^{2} \nabla^{2}}{2 m}+V_{e x t}(\mathbf{r})\right] w\left(\mathbf{r}-\mathbf{R}_{i}\right) d \mathbf{r}, \\
& U=g_{3 D} \int\left|w\left(\mathbf{r}-\mathbf{R}_{i}\right)\right|^{4} d \mathbf{r} .
\end{aligned}
$$

- J: Parâmetro associado à energia cinética, representa a energia que um átomo precisa para tunelar de um sítio a outro. Nesse caso um tunelamento isotrópico restrito aos primeiros vizinhos.

- U: Parâmetro associado à energia potencial, representa a energia de interação dos átomos em um mesmo sítio. Consideraremos interação repulsiva entre os átomos $(U>0)$. Tratando-se de bósons, podemos ter mais de um átomo por sítio, de acordo com o balanço entre as energias $U$ e o potencial químico do sistema.

\subsubsection{Aproximação gaussiana}

Para estimar valores analíticos para tunelamento e energia de interação, normalmente as funções de Wannier são definidas como funções gaussianas, com largura média muito menor do que o parâmetro de rede e superposição quase nula entre sítios vizinhos. Apesar de aproximar corretamente a energia de interação $U$, a onda gaussiana falha na aproximação do tunelamento $J$, já que a "cauda" da Wannier não é bem descrita.

Definindo o perfil gaussiano normalizado da função de Wannier unidimensional

$$
w_{i}(x)=\left(\frac{m \omega_{l}}{\pi \hbar}\right)^{1 / 4} e^{-\frac{m \omega_{l}}{2 \hbar}\left(x-x_{i}\right)^{2}}
$$


onde $w_{i}(x)=w\left(x-x_{i}\right)$ e $\omega_{l}$ frequência angular do potencial externo.

A Eq. (4.11) descreve a solução para o confinamento de um átomo em potencial de oscilador harmônico, o qual surge ao expandir o potencial periódico da rede em torno dos sítios. O comportamento do tunelamento é completamente descrito em forma unidimensional devido à isotropia do tunelamento entre vizinhos.

Considerando o potencial externo periódico em Eq. (3.1), substituindo a energia de recuo, Eq. (3.2), e a função de Wannier com perfil gaussiano, Eq. (4.11), no tunelamento, Eq. (4.8), obtém-se

$$
\begin{aligned}
J & =-\left(\frac{m \omega_{l}}{\pi \hbar}\right)^{1 / 2} \int_{-\infty}^{\infty+} e^{-\frac{m \omega_{l}}{2 \hbar}\left(x-x_{i}\right)^{2}}\left[-\frac{\hbar^{2} \nabla^{2}}{2 m}+V_{0} \cos ^{2}\left(k_{l} x\right)\right] e^{-\frac{m \omega_{l}}{2 \hbar}\left(x-x_{j}\right)^{2}} d x \\
& =-e^{-\frac{m \omega_{l}}{4 \hbar} d^{2}}\left[-\frac{\hbar \omega_{l}}{2}\left(\frac{1}{2}+\frac{\hbar \omega_{l}}{2} d^{2}\right)+V_{0}\left(1+e^{-\frac{\pi^{2} \hbar}{m \omega_{l} d^{2}}}\right)\right] \\
& =-e^{-\frac{\pi^{2}}{4} \sqrt{V_{0} / E_{R}}}\left[-\frac{\sqrt{V_{0} E_{R}}}{2}+\frac{V_{0}}{2}\left(1+e^{-\sqrt{E_{R} / V_{0}}}-\frac{\pi^{2}}{2}\right)\right]
\end{aligned}
$$

com $\hbar \omega_{l}=\sqrt{4 V_{0} E_{R}}$ e o parâmetro de rede definido como

$$
d=x_{i}-x_{j}
$$

Para redes profundas $V_{0} \gg E_{R}$, a equação (4.12) transforma-se em

$$
J=E_{R}\left(\frac{V_{0}}{E_{R}}\right)\left[\frac{\pi^{2}}{4}-1\right] e^{-\frac{\pi^{2}}{4} \sqrt{V_{0} / E_{R}}}
$$

A genealização para o caso do tunelamento na rede $3 D$ traz o somatório sobre o número de primeiros vizinhos.

Assumindo o potencial externo isotrópico $V(\mathbf{r})=V_{0}\left[\cos ^{2}\left(k_{l} x\right)+\cos ^{2}\left(k_{l} y\right)+\cos ^{2}\left(k_{l} z\right)\right]$, com frequências angulares equivalentes: $\omega_{x}=\omega_{y}=\omega_{z}=\omega_{l}$. De acordo com Eq. (4.10), a energia de interação $(3 D)$ será dada por

$$
\begin{aligned}
U & =\frac{4 \pi \hbar^{2} a_{s}}{m}\left[\frac{m \omega_{l}}{\pi \hbar} \int_{-\infty}^{\infty+} e^{-\frac{2 m \omega_{l}}{\hbar}\left(x-x_{i}\right)^{2}} d x\right]^{3} \\
& =\frac{4 \pi \hbar^{2} a_{s}}{m}\left[\frac{m \omega_{l}}{\pi \hbar} \sqrt{\frac{\pi \hbar}{2 m \omega_{l}}}\right]=\sqrt{\frac{8 a_{s}^{2} \pi}{E_{R} d^{2}}}\left(V_{0} E_{R}\right)^{3 / 4} \\
& =2 \sqrt{2 \pi}\left(\frac{a_{s}}{d}\right) E_{R}\left(\frac{V_{0}}{E_{R}}\right)^{3 / 4}
\end{aligned}
$$




\subsubsection{Equação de Mathieu}

A fim de estudar o comportamento das vibrações em membranas elípticas, no ano 1868, Émile Mathieu (66) propõe que elas são soluções da seguinte equação diferencial

$$
\frac{d^{2} h(y)}{d y^{2}}+[\alpha-2 \beta \cos (2 y)] h(y)=0
$$

onde $\alpha$ e $\beta$ são parâmetros arbitrários.

A solução dessa equação pode ser aplicada ao caso de potenciais periódicos em uma rede. (67-69) A equação de Schrödinger com potencial externo periódico pode ser reescrita na forma da Eq. (4.16):

$$
\left(-\frac{\hbar^{2}}{2 m} \frac{d^{2}}{d x^{2}}+V_{0} \cos ^{2}\left(k_{l} x\right)\right) \psi(x)=E \psi(x) \Rightarrow \frac{d^{2} \psi(x)}{\left(d k_{l} x\right)^{2}}+\left(\frac{2 m \bar{E}}{k_{l}^{2}}-\frac{m V_{0}}{k_{l}^{2}} \cos \left(2 k_{l} x\right)\right) \psi(x)=0,
$$

com

$$
h(y) \equiv \psi(x), \quad y=k_{l} x, \quad \alpha=\frac{2 m \bar{E}}{k_{l}^{2}}, \quad \beta=\frac{m V_{0}}{2 k_{l}^{2}} .
$$

sendo $k_{l}=2 \pi / \lambda, V_{0}$ profundidade da rede e $E$ autovalor da energia $\left(\bar{E}=E-V_{0} / 2\right)$.

Abramowitz e Stegun (70), usando a equação (4.17), derivaram a expressão para largura das bandas de energia

$$
A_{m+1}(\beta)-A_{m}(\beta) \approx \frac{2^{4 m+6}}{\sqrt{2 \pi} m !} \beta^{m / 2+3 / 4} e^{-4 \sqrt{\beta}}\left(1-\frac{6 m^{2}+14 m+7}{32 \sqrt{\beta}}\right),
$$

onde $m$ representa a banda de energia, com a distância entre elas dadas por $\Delta A_{m, m+1}=$ $A_{m+1}-A_{m}$. Em particular, para primeira banda de energia teremos

$$
\Delta A_{0,1}=A_{1}-A_{0} \approx \frac{64}{\sqrt{2 \pi}} \beta^{3 / 4} e^{-4 \sqrt{\beta}}\left(1-\frac{7}{32 \sqrt{\beta}}\right),
$$

onde $\beta=V_{0} / 4 E_{R}$ e $\Delta A_{0,1}=4 J / E_{R}(10,53)$, de acordo com as Eqs. (3.2) e (4.18).

Para o limite de rede profunda $V_{0} \gg E_{R}, 1 / \beta \rightarrow 0$. Com o auxílio da Eq. (4.20), deriva-se a solução exata para o tunelamento referente à menor banda de energia $(11,71)$

$$
J_{M} \approx \frac{4}{\sqrt{\pi}} E_{R}\left(\frac{V_{0}}{E_{R}}\right)^{3 / 4} e^{-2 \sqrt{V_{0} / E_{R}}} .
$$

Essa equação será utilizada para determinar o ponto crítico da transição de fase quântica. 


\subsection{Transição de fase quântica}

Quando o estado fundamental de um sistema físico muda abruptamente devido às flutuações quânticas, diz-se que o sistema atingiu a TFQ (no caso clássico essa transição deve-se às flutuações térmicas). Para $T=0 \mathrm{~K}$, as mudanças do sistema são puramente quânticas. (57) A partir do BHM é possível obter a TFQ analisando a competição entre as energias de tunelamento $(J)$ e de interação $(U)$; justamente devida à essa competição o sistema é induzido a ter mais de um estado fundamental. O limite termodinâmico fixa o sistema em um desses estados fundamentais. O ponto crítico da TFQ em geral acontece quando a razão das energias $(U / J)$ é da ordem 1. (71)

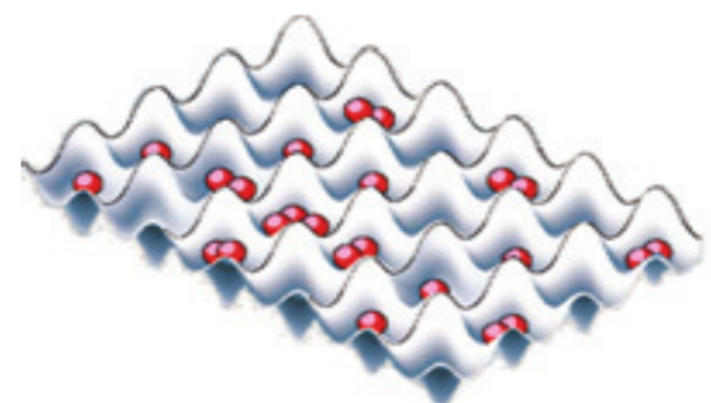

(a)

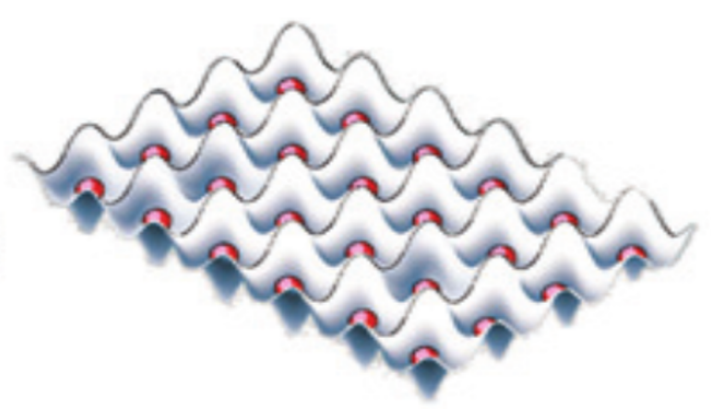

(b)

Figura 11 - Rede óptica com fator de preenchimento $\nu=1$. (a) Fase superfluida, os átomos estão delocalizados. (b) Fase isolante de Mott, os átomos estão localizados.

Fonte: LEVIN; FETTER; STAMPER-KURN. (72)

\section{- Fase superfluida (SF)}

Quando a energia de tunelamento predomina sobre a energia de interação $(J \gg U)$. O sistema minimiza sua energia no processo de delocalizar os átomos na rede (Figura 12a). O tunelamento dos átomos entre sítios vizinhos mantém a coerência na fase, de forma que os átomos compartilham o mesmo estado quântico. Desse modo eles são descritos pela mesma função de onda individual do estado fundamental: $\left|\psi_{\text {indiv }}\right\rangle=\frac{1}{\sqrt{N_{s}}} \sum_{i=1}^{N_{s}} \hat{a}_{i}^{\dagger}|0\rangle$, normalizada com respeito ao número de sítios $N_{s}$.

O estado SF dos $N$ átomos corresponde ao produtório das funções de onda individuais:

$$
\left|\psi_{S F}\right\rangle=\frac{1}{\sqrt{N !}}\left(\left|\psi_{\text {indiv }}\right\rangle\right)^{N}=\frac{1}{\sqrt{N !}}\left(\frac{1}{\sqrt{N_{s}}} \sum_{i=1}^{N_{s}} \hat{a}_{i}^{\dagger}\right)^{N}|0\rangle .
$$




\section{- Fase Isolante de Mott (MI)}

Quando a energia de interação prevalece sobre a energia de tunelamento $(U \gg J)$. O sistema minimiza sua energia total reduzindo o número médio de átomos em cada sítio, favorecendo dessa forma a distribuição dos átomos por toda a rede (Fig. 12b). O processo de tunelamento é suprimido fazendo com que a fase do sistema não esteja bem definida, e que os átomos apresentem máxima localização nos sítios da rede.

O estado fundamental dos $N$ átomos localizados é descrito pela função de onda de muitos corpos de Fock:

$$
\left|\psi_{M I}\right\rangle \propto \prod_{i=1}^{N_{s}}\left(\hat{a}_{i}^{\dagger}\right)^{m}|0\rangle, \quad m \in \mathbb{Z}^{+}
$$

onde $m$ é o número de átomos por sítio.

O surgimento da fase MI é devido às fortes correlações que acontecem na rede como consequência das interações interatômicas nos sítios. Neste caso os átomos precisam de certa energia (gap) para transportar-se de um sítio para outro.

\subsubsection{Aproximação de mean-field}

As fortes correlações atômicas advindas das interações interatômicas impossibilitam soluções analíticas para o Hamiltoniano de BH, Eq. (4.7). Existem técnicas aproximadas, porém, que permitem simplificar essas correlações, viabilizando as soluções analíticas, sem perder a natureza física do problema. (73) Um desses métodos, chamado de mean-field de desacoplamento, ou simplesmente mean-field (MF), consiste em rescrever os operado-

res $\hat{a}_{i}$ e $\hat{a}_{i}^{\dagger}$ em termos de seus valores médios e uma flutuação. Como será mostrado na sequência, essa transformação diagonaliza o Hamiltoniano.

Seja a transformação dos operadores

$$
\hat{a}_{i}=\left\langle\hat{a}_{i}\right\rangle+\delta \hat{a}_{i}=\phi_{i}+\delta \hat{a}_{i} \quad \text { e } \quad \hat{a}_{i}^{\dagger}=\left\langle\hat{a}_{i}^{\dagger}\right\rangle+\delta \hat{a}_{i}^{\dagger}=\phi_{i}^{*}+\delta \hat{a}_{i}^{\dagger}
$$


onde $\phi_{i}=\left\langle\hat{a}_{i}\right\rangle$ representa o parâmetro de ordem e $\delta \hat{a}_{i}$ a flutuação. De forma que

$$
\begin{aligned}
\hat{a}_{i}^{\dagger} \hat{a}_{j} & =\left(\phi_{i}^{*}+\delta \hat{a}_{i}^{\dagger}\right)\left(\phi_{j}+\delta \hat{a}_{j}\right) \\
& =\phi_{i}^{*} \phi_{j}+\phi_{i}^{*}\left(\hat{a}_{j}-\phi_{j}\right)+\phi_{j}\left(\hat{a}_{i}^{\dagger}-\phi_{i}^{*}\right)+\delta \hat{a}_{i}^{\dagger} \delta \hat{a}_{j} \\
& \approx \phi_{i}^{*} \hat{a}_{j}+\phi_{j} \hat{a}_{i}^{\dagger}-\phi_{i}^{*} \phi_{j},
\end{aligned}
$$

onde o termo de segunda ordem da flutuação foi negligenciado.

Substituindo a equação (4.23) em (4.7), consegue-se o Hamiltoniano de mean-field

$$
\hat{H}_{B H}^{M F}=-J \sum_{\langle i j\rangle}\left(\phi_{i}^{*} \hat{a}_{j}+\phi_{i} \hat{a}_{j}^{\dagger}-\phi_{i}^{*} \phi_{i}\right)-\mu \sum_{i} \hat{n}_{i}+\frac{U}{2} \sum_{i} \hat{n}_{i}\left(\hat{n}_{i}-1\right) .
$$

A somatória em $\langle i j\rangle$ pode ser expandida introduzindo o fator $z$ associado aos primeiros vizinhos. Dessa forma

$$
\sum_{\langle i j\rangle} \phi_{i}^{*} \hat{a}_{j}=z \sum_{i} \phi_{i}^{*} \hat{a}_{i} \quad \text { e } \sum_{\langle i j\rangle} \phi_{i}^{*} \phi_{j}=z \sum_{i}\left|\phi_{i}\right|^{2}
$$

reduzindo a somatória para um sítio.

Sem perder generalidade, seja $\phi_{i}=\phi_{i}^{*}=\phi$. Substituindo a Eq. (4.25) em (4.24), tem-se

$$
\begin{aligned}
\hat{H}_{B H}^{M F} & =-J z \sum_{i}\left(\phi \hat{a}_{i}+\phi \hat{a}_{i}^{\dagger}-\phi_{i}^{2}\right)-\mu \sum_{i j} \hat{n}_{i}+\frac{U}{2} \sum_{i} \hat{n}_{i}\left(\hat{n}_{i}-1\right) \\
& =\sum_{i}\left[-J z \phi\left(\hat{a}_{i}+\hat{a}_{i}^{\dagger}\right)\right]+\sum_{i}\left[z J \phi_{i}^{2}+\hat{H}_{i}^{0}\right] \\
& =\sum_{i}\left[\phi \hat{V}_{i}+\overline{\hat{H}}_{i}^{0}\right] .
\end{aligned}
$$

Onde o Hamiltoniano de mean-field corresponde ao somatório dos Hamiltonianos de BH de cada sítio

$$
\hat{H}_{i}=\phi \hat{V}_{i}+\overline{\hat{H}}_{i}^{0}
$$

com

$$
\begin{aligned}
\hat{V}_{i} & =-z J\left(\hat{a}_{i}^{\dagger}+\hat{a}_{i}\right), \\
\overline{\hat{H}}_{i}^{0} & =\hat{H}_{i}^{0}+z J \phi^{2}=-\mu \hat{n}_{i}+\frac{U}{2} \hat{n}_{i}\left(\hat{n}_{i}-1\right)+z J \phi^{2} .
\end{aligned}
$$




\subsubsection{Teoria de perturbações de Rayleigh-Schrödinger}

Considerando o Hamiltoniano do sítio na Eq. (4.27), fazendo $\hat{n}_{i} \rightarrow \hat{n}$, por simplicidade, seja a equação de Schrödinger para as auto-energias

$$
E_{n}^{M F}|n\rangle=\hat{H}_{n}|n\rangle=\left(\phi \hat{V}+\overline{\hat{H}}_{n}^{0}\right)|n\rangle,
$$

onde $\hat{V}$ será considerado como uma perturbação sobre o Hamiltoniano $\hat{H}^{0}$. Expandindo essa equação explicitamente até segunda ordem da perturbação, tem-se

$$
E_{n}^{M F} \approx \bar{E}_{n}^{0}+\phi\langle n|\hat{V}| n\rangle+\phi^{2} \sum_{m \neq n} \frac{|\langle m|\hat{V}| n\rangle|^{2}}{\left(\bar{E}_{n}^{0}-\bar{E}_{m}^{0}\right)}+\mathcal{O}\left(\phi^{3}\right) .
$$

Utilizando a álgebra dos operadores de escada e do operador número:

$$
\begin{aligned}
\hat{a}^{\dagger}|n\rangle & =\sqrt{n+1}|n+1\rangle \\
\hat{a}|n\rangle & =\sqrt{n}|n-1\rangle \\
\hat{n}|n\rangle & =n|n\rangle,
\end{aligned}
$$

teremos para o segundo e terceiro termos da Eq. (4.30)

$$
\begin{aligned}
\langle n|\hat{V}| n\rangle & =\left\langle n\left|-z J\left(\hat{a}^{\dagger}+\hat{a}\right)\right| n\right\rangle=0 \\
|\langle m|\hat{V}| n\rangle|^{2} & =(z J)^{2}\left|\left\langle m\left|\hat{a}^{\dagger}\right| n\right\rangle+\langle m|\hat{a}| n\rangle\right|^{2} \\
& =(z J)^{2}\left[n \delta_{m, n-1}+(n+1) \delta_{m, n+1}+\sqrt{n(n+1)}\left(\delta_{m, n+1}+\delta_{m, n-1}\right)\right], \\
\left\{\begin{array}{l}
\bar{E}_{n}^{0}|n\rangle=\overline{\hat{H}} \\
\bar{E}_{n}^{0}=\frac{U}{2} n(n-1)-\mu n+z J \phi^{2}
\end{array} \Rightarrow E_{n}^{0}+z J \phi^{2}\right) & \Rightarrow \bar{E}_{m}^{0}-\bar{E}_{n}^{0}=\frac{U}{2}[n(n-1)-m(m-1)]+\mu(n-m) .
\end{aligned}
$$

Considerando os autovalores do problema não perturbado, Eq. (4.33), obtem-se finalmente $E_{n}^{M F} \approx E_{n}^{0}+\phi^{2}\left[z J+(z J)^{2} \sum_{m \neq n}\left(\frac{n \delta_{m, n-1}+(n+1) \delta_{m, n-1}+2 \sqrt{n(n+1)} \delta_{m, n+1} \delta_{m, n-1}}{\frac{U}{2}[n(n-1)-m(m-1)]+\mu(n-m)}\right)\right]+\mathcal{O}\left(\phi^{3}\right)$.

Desta forma, a energia do estado fundamental na aproximação de mean-field até segunda ordem da teoria de perturbações será dada por

$$
E_{n}^{M F} \approx E_{n}^{0}+\phi^{2}\left[z J+(z J)^{2}\left(\frac{n}{U(n-1)-\mu}-\frac{n+1}{U n-\mu}\right)\right]+\mathcal{O}\left(\phi^{3}\right) .
$$


Por outro lado, empregando a aproximação de Landau para a transição de fase, o mínimo de energia que estabelece o estado de equílibrio do sistema, pode ser obtido expandindo a energia do sistema em termos do parâmetro de ordem

$$
E_{n}^{M F}=a_{0}(n, U, \mu)+a_{2}(n, U, \mu) \phi^{2}+\mathcal{O}\left(\phi^{4}\right)
$$

Quando $a_{2}(n, U, \mu)=0(12)$, o sistema atinge a TFQ. Comparando os coeficientes dos termos quadráticos em $\phi$ nas Eqs. (4.34) e (4.35), usando a condição da TFQ, obtém-se

$$
1+z J\left(\frac{n}{U(n-1)-\mu}-\frac{n+1}{U n-\mu}\right)=0 .
$$

Reescrevendo a Eq. (4.36) em termos dos parâmetros adimensionais $\bar{U}=U / z J$ e $\bar{\mu}=\mu / z J$, deduz-se a equação dos lóbulos

$$
\left(\frac{n}{\bar{U}(n-1)-\bar{\mu}}+\frac{n+1}{\bar{\mu}-\bar{U} n}\right)=-1
$$

Resolvendo a equação (4.37), tem-se: $\bar{\mu}_{1,2}=\frac{1}{2}[\bar{U}(2 n-1)-1] \pm \frac{1}{2} \sqrt{\bar{U}^{2}-2 \bar{U}(2 n+1)+1}$, onde o valor crítico para transição de fase é alcançado quando $\bar{\mu}_{1}=\bar{\mu}_{2}$, de forma que

$$
\bar{U}_{c}=(2 n+1) \pm \sqrt{(2 n+1)^{2}-1} \quad \text { para todo } n
$$

Para o caso $n=1$, primeiro lóbulo $(12,74,75)$, tem-se

$$
\bar{U}_{c}=3+\sqrt{2} \approx 5.83 \quad \longrightarrow \quad(U / J)_{c}=5.83 z
$$

Considerando a aproximação gaussiana feita nas Eq. (4.15) e Eq. (4.21), o ponto crítico toma a forma

$$
\left(\frac{U}{J_{M}}\right)_{c}=\frac{\pi}{\sqrt{2}}\left(\frac{a_{s}}{d}\right) e^{2 \sqrt{V_{0} / E_{R}}} \approx 5.83 z .
$$

\subsubsection{Diagrama de fases}

Para $T=0 \mathrm{~K}$, o diagrama de fases, Figura 12, mostra uma série de curvas conhecidas como lóbulos de Mott. Esses lóbulos comportan-se como fronteira entre as fases isolante de 
Mott (dentro dos lóbulos) e a superfluida (fora dos lóbulos). Cada lóbulo da fase MI tem uma densidade (número de átomos por sítio) limitada por um número inteiro (ocupação, $n=1,2, \ldots)$; além disso, a fase MI possui uma comprensibilidade nula.

A energia gap, ou energia para criar excitações na correspondente fase de MI, pode ser obtida computando as distâncias ente os lóbulos superior e inferior $\left(\mu_{+}\right.$e $\left.\mu_{-}\right)$a partir de um certo valor de $J$.

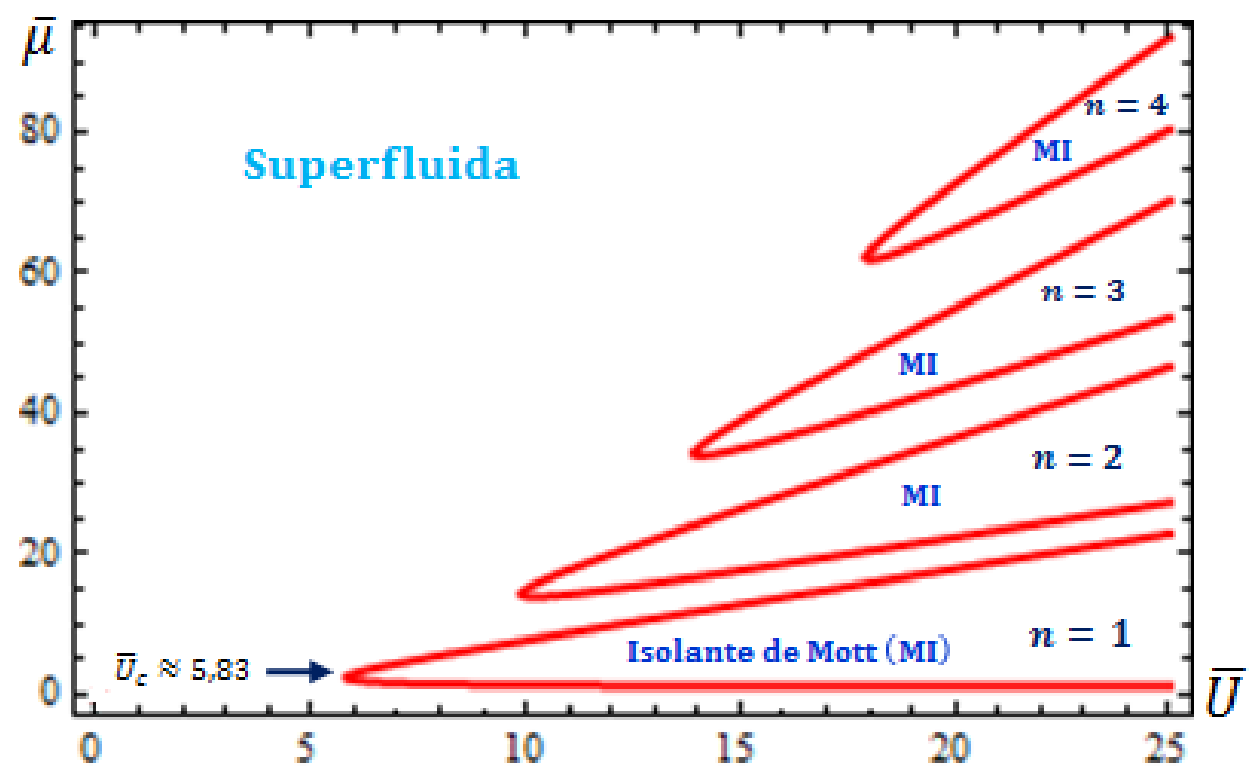

Figura 12 - Gráfico da equação (4.37) $\operatorname{com} \bar{\mu}=\mu / z J$ e $\bar{U}=U / z J$. A área correspondente à fase de isolante de Mott varia de acordo com o número de ocupação dos sítios da rede.

Fonte: Elaborada pelo autor. 



\section{Rede de vórtices estática}

Neste capítulo apresenta-se o modelo teórico das espécies atômicas interagindo em um sistema bidimensional. A descrição matemática da interação entre elas e sua relação com o modelo das redes ópticas será detalhado na sequência.

\subsection{Regimes do estudo}

Antes de descrever o modelo das duas espécies, definimos o regime de estudo apropriado para aplicação das ferramentas teóricas e com viabilidade experimental.

- Mean-field: Esse regime restringe a escala de comprimentos do sistema de duas espécies, i.e. garante que os parâmetros associados à dimensão física da rede de vórtices sejam muito maiores que os comprimentos de espalhamento (onda-s) das espécies atômicas.

$$
a_{A}, a_{B}, a_{A B} \ll \xi \leqslant d,
$$

onde $\xi$ e $d$ representam o healing length e a distância entre os vórtices respectivamente. A ordem dos espalhamentos $a_{A, B, A B} \sim 10^{-9} \mathrm{~m}$, enquanto que $d, \xi \sim 10^{-6} \mathrm{~m}$.

- Menor banda de energia de Bloch: Regime associado com a energia de interação entre as duas espécies. Considera-se os átomos da espécie $B$ restritos à menor banda de energia de Bloch, de modo que possam ser descritos através do limite de tight-binding. Essa condição indiretamente permite aplicar a aproximação do tunelamento entre primeiros vizinhos. No regime de tight-binding, tem-se

$$
V_{0} \gg E_{R}
$$

onde $V_{0}$ e $E_{R}$ se associam ao potencial de aprisionamento da espécie $B$ na rede de vórtices da espécie $A$. Tanto $V_{0}$ como $E_{R}$ serão computados em termos dos parâmetros intrínsecos das espécies atômicas. 
- Condição de quase-2D: Essa condição está associada com as dimensões espaciais do condensado (espécie $A$ )

$$
l_{\rho_{e f}} \gg l_{z}
$$

onde $l_{z}$ e $l_{\rho_{e f}}$ são os confinamentos axial e radial efetivo.

Esse regime garante um sistema bidimensional. O comprimento axial pode ser variado através da frequência de confinamento na direção axial, Eq. (5.8e). É importante ressaltar que a ordem de grandeza do confinamento axial $l_{z} \sim 10^{-6} \mathrm{~m}$.

- Lowest-Landau-Level (LLL): Também conhecido como MF-LLL. Esse regime também está relacionado com a menor banda de energia de Bloch, de acordo com (42)

$$
\Gamma=\frac{g_{A} n_{A}}{2 \hbar \Omega}=\frac{d^{2}}{16 \xi^{2}}<1
$$

onde $\mu_{A}=g_{A} n_{A}$ é o potencial químico da espécie $A$ e $\Omega\left[=n_{v} \pi \hbar / m\right.$, Eq. (2.29), sendo $n_{v}$ a densidade bidimensional dos vórtices] é a frequência de excitação do BEC.

O regime MF-LLL pode ser dado em termos do fator $\bar{\Omega}$, Eq. (2.27). O aumento gradativo da rotação $\left(\Omega \rightarrow \omega_{\rho}, \bar{\Omega} \rightarrow 1\right)$, além de induzir o aumento do número de vórtices, faz com que o BEC expanda-se radialmente, trazendo a diminuição da densidade atômica no centro. Os vórtices gerados irão se distribuir pela área disponível do condensado, com interação repulsiva entre eles (vórtices de mesma circulação) direcionando a formação da estrutura da rede triangular. $\Gamma$ e $\bar{\Omega}$ podem ser associados assim (42)

- Quando $0.99 \lesssim \bar{\Omega} \lesssim 0.999 \Rightarrow \Gamma=\Gamma_{L L L}<1$.

O caso de interesse foi atingido experimentalmente por Schweikhard (76), com $\Gamma_{L L L} \approx 0.6$.

\subsubsection{Características das espécies para $T \approx 0 K$}

O sistema de estudo consiste de uma espécie majoritária (espécie $A$ ) e uma minoritária (espécie $B$ ).

- A: superfluido (BEC) $2 D$ composto de uma rede triangular de vórtices.

- B: gás diluído ultrafrio comportando-se como impurezas na rede de vórtices. 
Uma das característica desse estudo será limitar o número de átomos da espécie $B$ com respeito ao número de vórtices da espécie $A$. Como os vórtices gerados dependem da excitação externa $\Omega$ e a espécie $B$ comporta-se como uma impureza, é factível assumir que as ordens entre estas grandezas sejam equivalentes, i.e. $N_{v} \sim N_{B}$.

Por outro lado, considerando a condição de "high filling factor", temos que $N_{B} \ll N_{A}$. Além de garantir um preenchimento unitário $(\nu=1)$, esta restrição permite desconsidermos deformações na rede devido à interação com os àtomos da espécie $B$, garantindo a estabilidade desse sistema.

Fazendo analogia com a rede óptica, a rede de vórtices corresponderia a uma rede triangular $2 D$, onde os vórtices representam os potenciais periódicos de aprisionamento, i.e. os sítios da rede. Nesse cenário a interação dos átomos da espécie $B$ com a rede $A$ pode ser descrita através do modelo de Bose-Hubbard, conforme será descrito na sequência.

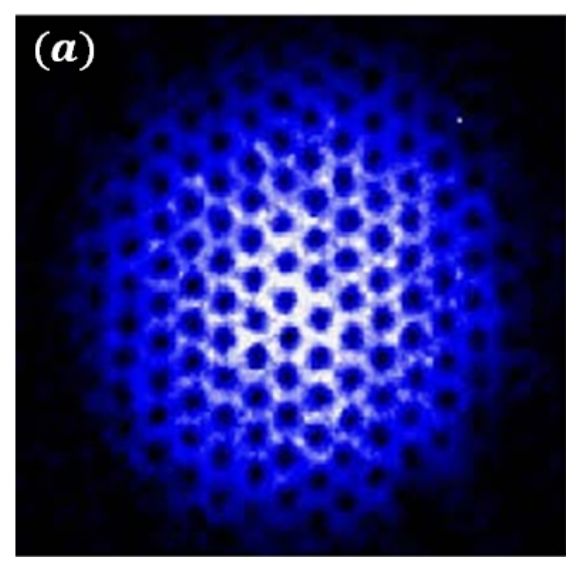

Figura 13 - Imagem de absorção (perfil de densidade) da espécie $A$ composta de um arranjo triangular de vórtices.

Fonte: CORNELL. (38)

\subsection{Hamiltoniano do sistema 2D}

A aproximação do Hamiltoniano $2 D$ é obtida considerando que a frequência efetiva do confinamento radial na Eq. (2.26) tende a zero, i.e. $\omega_{\rho} \rightarrow \Omega$. Essa condição pode ser 
obtida tanto para o regime de MF-TF como para o MF-LLL. Desse modo o Hamiltoniano 3D da Eq. (2.23) fica

$$
H \approx \frac{(\mathbf{p}-\mathbf{A})^{2}}{2 m}+\frac{m}{2} \omega_{z}^{2} z^{2}=H_{2 D}+\frac{m}{2} \omega_{z}^{2} z^{2}
$$

onde o termo associado ao eixo $\hat{z}$ pode ser tomado como uma frequência perpendicular ao plano do Hamiltoniano $2 D$.

Para descrever corretamente a rede de vórtices devemos incluir a interação entre os átomos, de forma que a equação (5.5) escrita na linguagem de segunda quantização fica

$$
\hat{H}_{2 D}=\int\left[\hat{\psi}^{\dagger}(\mathbf{r}) \frac{(i \hbar \nabla-\mathbf{A})^{2}}{2 m} \hat{\psi}(\mathbf{r}) d \mathbf{r}+\frac{g_{2 D}}{2} \hat{\psi}^{\dagger}(\mathbf{r}) \hat{\psi}^{\dagger}(\mathbf{r}) \hat{\psi}(\mathbf{r}) \hat{\psi}(\mathbf{r})\right] d \mathbf{r} \quad \text { com } \quad \mathbf{r} \in \perp \mathrm{xy},
$$

onde $\hat{\psi}^{\dagger}(\mathbf{r}), \hat{\psi}(\mathbf{r})$ são os operadores de campo e $g_{2 D}$ é o potencial efetivo de contato.

No regime MF-LLL, o Hamiltoniano (5.6) descreve completamente a rede triangular de vórtices do condensado, com solução analítica para o estado fundamental [análise com variáveis complexas $(77,78)]$

$$
\phi(z)=\sqrt{n} \varphi(z)
$$

$\operatorname{com} \varphi(z)=(2 v)^{1 / 4} \vartheta_{1}(\sqrt{\pi v} z, q) e^{z / 2} e^{-|z|^{2} / 2}$ sendo $\vartheta_{1}$ a função de Jacobi e $q=e^{i \pi \tau}, \tau=$ $u+i v, v=\sqrt{3} / 2, u=-1 / 2, z=x+i y$ e $n$ é a densidade atômica em $2 D$.

O sistema das duas espécies fica completamente caracterizado através do Hamiltoniano (5.6) $\operatorname{com}(79)$

$$
\begin{aligned}
\hat{H}_{A} & =\int\left[\hat{\psi}_{A}^{\dagger}(\mathbf{r}) \frac{(-i \hbar \nabla-\mathbf{A})^{2}}{2 m_{A}} \hat{\psi}_{A}(\mathbf{r})+\frac{g_{A}}{2} \hat{\psi}_{A}^{\dagger}(\mathbf{r}) \hat{\psi}_{A}^{\dagger}(\mathbf{r}) \hat{\psi}_{A}(\mathbf{r}) \hat{\psi}_{A}(\mathbf{r})\right] d \mathbf{r} \\
\hat{H}_{B} & =\int\left[\hat{\psi}_{B}^{\dagger}(\mathbf{r}) \frac{(-i \hbar \nabla)^{2}}{2 m_{B}} \hat{\psi}_{B}(\mathbf{r})+\frac{g_{B}}{2} \hat{\psi}_{B}^{\dagger}(\mathbf{r}) \hat{\psi}_{B}^{\dagger}(\mathbf{r}) \hat{\psi}_{B}(\mathbf{r}) \hat{\psi}_{B}(\mathbf{r})\right] d \mathbf{r} \\
\hat{H}_{A B} & =g_{A B} \int \hat{\psi}_{A}^{\dagger}(\mathbf{r}) \hat{\psi}_{B}^{\dagger}(\mathbf{r}) \hat{\psi}_{A}(\mathbf{r}) \hat{\psi}_{B}(\mathbf{r}) d \mathbf{r}
\end{aligned}
$$

com os potencias efetivos de contato em $2 \mathrm{D}$

$$
\begin{aligned}
g_{i} & =2 \sqrt{2 \pi} a_{i} \hbar^{2} / m_{i} l_{z}, \quad g_{A B}=\sqrt{2 \pi} a_{i j} \hbar^{2} / m_{r} l_{z}, \quad i, j=A, B, \\
m_{r} & =\frac{m_{A} m_{B}}{m_{A}+m_{B}}, \quad l_{z}=\sqrt{\frac{\hbar}{m_{A} \omega_{z}}}, \quad l_{\rho_{e f}}=\sqrt{\frac{\hbar}{m_{A} \omega_{e f}}},
\end{aligned}
$$

com $m_{A}$ e $m_{B}$ sendo as massas das espécies $A$ e $B$; o comprimento $l_{z}$ representa o confinamento axial do condensado; $a_{A}, a_{B}$ representam os comprimentos de espalhamento de 
onda-s das espécies $A$ e $B$, enquanto que $a_{A B}$ é o comprimento de espalhamento entre as espécies.

A mistura é descrita usando o formalismo grande-canônico, i.e., mantendo o número de átomos invariante com a inclusão do potencial químico

$$
\hat{\mathcal{H}}=\hat{\mathcal{H}}_{\mathcal{A}}+\hat{\mathcal{H}}_{\mathcal{B}}+\hat{H}_{A B}=\hat{H}_{A}-\mu_{A} \hat{N}_{A}+\hat{H}_{B}-\mu_{B} \hat{N}_{B}+\hat{H}_{A B}
$$

com

$$
\hat{N}_{A}=\int \hat{\psi}_{A}^{\dagger}(\mathbf{r}) \hat{\psi}_{A}(\mathbf{r}) d \mathbf{r}, \quad \hat{N}_{B}=\int \hat{\psi}_{B}^{\dagger}(\mathbf{r}) \hat{\psi}_{B}(\mathbf{r}) d \mathbf{r}
$$

sendo os operadores número e $\mu_{A}, \mu_{B}$ os potenciais químicos das espécies $A$ e $B$.

\subsubsection{Espécie B: Limite de tight-binding}

Os átomos da espécie B comportam-se como impurezas aprisionadas nos vórtices da rede, restritas à menor banda de Bloch. Desse modo o operador de campo $\hat{\psi}_{B}$ pode ser expandido em termos das funções de Wannier. Adaptando a equação (4.4), têm-se

$$
\hat{\psi}_{B}(\mathbf{r})=\sum_{i} w\left(\mathbf{r}-\mathbf{R}_{i}\right) \hat{b}_{i} \quad \text { e } \quad \hat{\psi}_{B}^{\dagger}(\mathbf{r})=\sum_{i} w^{*}\left(\mathbf{r}-\mathbf{R}_{i}\right) \hat{b}_{i}^{\dagger}
$$

Empregando a Eq. (3.13), obtemos $\int|w|^{2} d \mathbf{r}=1$ com $w_{i}=w\left(\mathbf{r}-\mathbf{R}_{i}\right)$. Substituindo a equação (5.10) na equação (5.8b) tem-se o Hamiltoniano do sistema grande-canônico

$$
\begin{aligned}
\hat{\mathcal{H}}_{B}=\sum_{i j}\left[\int w_{i}^{*} \frac{-\hbar^{2} \nabla^{2}}{2 m_{B}} w_{j} d \mathbf{r}\right] \hat{b}_{i}^{\dagger} \hat{b}_{j}+\frac{g_{B}}{2} \sum_{i j k l} & {\left[\int w_{i}^{*} w_{k}^{*} w_{j} w_{l} d \mathbf{r}\right] \hat{b}_{i}^{\dagger} \hat{b}_{k}^{\dagger} \hat{b}_{j} \hat{b}_{l} } \\
& -\mu_{B} \sum_{i j}\left[\int w_{i}^{*} w_{j} d \mathbf{r}\right] \hat{b}_{i}^{\dagger} \hat{b}_{j} .
\end{aligned}
$$

Substituindo a equação (5.10) em conjunto com o operador de campo da espécie $A$, Eq. (2.12), reescrito como $\hat{\psi}_{A}(\mathbf{r}) \approx \phi_{A}(\mathbf{r})$ no Hamiltoniano de interação $\hat{H}_{A B}$, consegue-se

$$
\hat{H}_{A B}=\sum_{i j}\left[g_{A B} n_{A} \int w_{i}^{*}\left|\varphi_{A}(\mathbf{r})\right|^{2} w_{j} d \mathbf{r}\right] \hat{b}_{i}^{\dagger} \hat{b}_{j}
$$

Nessa aproximação de mean-field para a espécie $A$, com a expansão de Bloch para especie $B$, precisamos do perfil de densidade do sítios para caracterizar as Wannier localizadas. 


\subsection{Hamiltoniano de Bose-Hubbard}

Substituindo os Hamiltonianos da espécie $B$ e de interação, Eqs. (5.11a) e (5.11b), na relação $(5.9 \mathrm{a})$

$$
\begin{array}{r}
\hat{\mathcal{H}}_{B H}=\sum_{i j} \int w_{i}^{*}\left[\frac{-\hbar^{2} \nabla^{2}}{2 m_{B}}+g_{A B} n_{A}\left|\varphi_{A}(\mathbf{r})\right|^{2}\right] w_{j} d \mathbf{r} \hat{b}_{i}^{\dagger} \hat{b}_{j}-\mu_{B} \sum_{i j}\left[\int w_{i}^{*} w_{j} d \mathbf{r}\right] \hat{b}_{i}^{\dagger} \hat{b}_{j} \\
+\frac{g_{B}}{2} \sum_{i j k l}\left[\int w_{i}^{*} w_{k}^{*} w_{j} w_{l} d \mathbf{r}\right] \hat{b}_{i}^{\dagger} \hat{b}_{k}^{\dagger} \hat{b}_{j} \hat{b}_{l}+E_{A} .
\end{array}
$$

Com a aproximação de primeiros vizinhos, e considerando o termo da energia da espécie $A$ como simples deslocamento, obtém-se

$$
\begin{array}{r}
\hat{\mathcal{H}}_{B H} \approx \sum_{\langle i j\rangle} \int w_{i}^{*}\left[\frac{-\hbar^{2} \nabla^{2}}{2 m_{B}}+g_{A B} n_{A}\left|\varphi_{A}(\mathbf{r})\right|^{2}\right] w_{j} d \mathbf{r} \hat{b}_{i}^{\dagger} \hat{b}_{j}+\frac{g_{B}}{2} \sum_{i} \int\left|w_{i}\right|^{4} d \mathbf{r} \hat{n}_{i}\left(\hat{n}_{i}-1\right) \\
+\sum_{i} \int w_{i}^{*}\left[\frac{-\hbar^{2} \nabla^{2}}{2 m_{B}}+g_{A B} n_{A}\left|\varphi_{A}(\mathbf{r})\right|^{2}-\mu_{B}\right] w_{i} d \mathbf{r} \hat{n}_{i} .
\end{array}
$$

Assim

$$
\hat{\mathcal{H}}_{B H} \approx-J \sum_{\langle i j\rangle} \hat{b}_{i}^{\dagger} \hat{b}_{j}+\frac{U}{2} \sum_{i} \hat{n}_{i}\left(\hat{n}_{i}-1\right)-\mu \sum_{i} \hat{n}_{i}
$$

com

$$
\begin{aligned}
& J=-\int w_{i}^{*}\left[\frac{-\hbar^{2} \nabla^{2}}{2 m_{B}}+g_{A B} n_{A}\left|\varphi_{A}(\mathbf{r})\right|^{2}\right] w_{j} d \mathbf{r} . \\
& U=g_{B} \int\left|w_{i}\right|^{4} d \mathbf{r} . \\
& \mu=\mu_{B}-\int w_{i}^{*}\left[\frac{-\hbar^{2} \nabla^{2}}{2 m_{B}}+g_{A B} n_{A}\left|\varphi_{A}(\mathbf{r})\right|^{2}\right] w_{i} d \mathbf{r},
\end{aligned}
$$

onde $J, U$ e $\mu$ representam respectivamente o tunelamento, a energia de interação e o potencial químico deslocado. $\hat{n}_{i}\left(=\hat{b}_{i}^{\dagger} \hat{b}_{i}\right)$ é o operador número da espécie $B$.

\subsubsection{Aproximação gaussiana}

Para definirmos uma expressão analítica para função de Wannier localizada, é razoável expandir o potencial de interação ao redor do sítio da rede de vórtices (80)

$$
V(\mathbf{r})=g_{A B} n_{A}\left|\varphi_{A}(\mathbf{r})\right|^{2}=g_{A B} n_{A} \frac{r^{2}}{r^{2}+2 \xi^{2}},
$$


onde o healing length, Eq.(2.20), é redefinindo como

$$
\xi=\frac{\hbar}{\sqrt{2 m_{A} g_{A} n_{A}}} .
$$

Expandindo esse potencial em termos de $r / \xi$, obtêm-se os seguintes domínios

$$
V(\mathbf{r}) \approx \begin{cases}V_{0} \frac{r^{2}}{2 \xi^{2}} ; & r \ll \xi \\ g_{A B} n_{A}=V_{0} ; & r \gg \xi .\end{cases}
$$

- Quando $r \ll \xi$ : tem-se o potencial de um oscilador harmônico. Conforme será mostrado, a função de Wannier será caracterizada em termos dos parâmetros dessa aproximação.

- Quando $r \gg \xi$ : O potencial comporta-se como uma barreira, com a interação entre dois sítios vizinhos será limitada por este potencial.

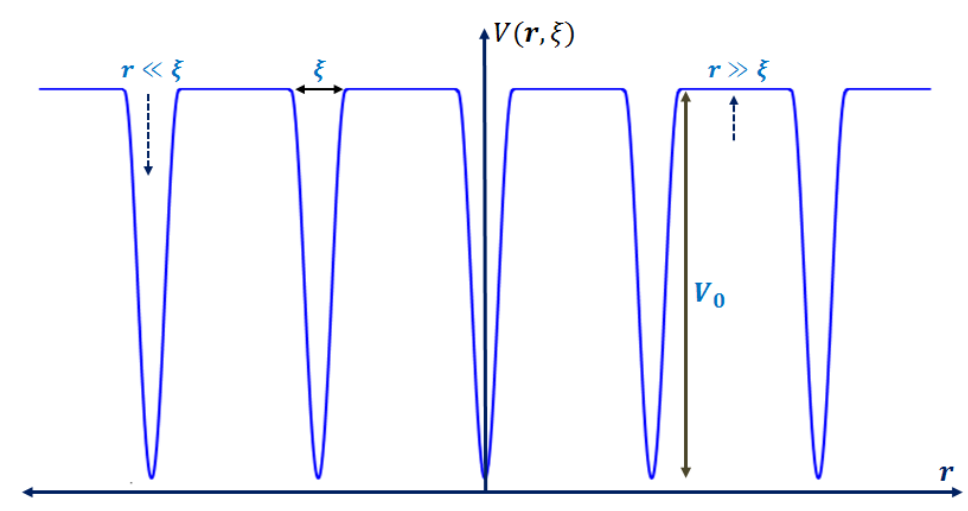

Figura 14 - Variação do potencial local do sítio (vórtices) nos limites extremos da relação: $r / \xi$. Fonte: Elaborada pelo autor.

- Potencial harmônico: comparando-se o perfil do sítio $V(r)=\frac{V_{0}}{2} \xi^{2}\left(x^{2}+y^{2}\right)$ com $V(r)=\frac{1}{2} m_{B}\left(\omega_{0 x}^{2} x^{2}+\omega_{0 y}^{2} y^{2}\right)$, obtém-se as frequências

$$
\omega_{0 x}=\omega_{0 y}=\omega_{0}=\sqrt{\frac{V_{0}}{m_{B} \xi^{2}}} .
$$

Os comprimentos de oscilador correspondentes

$$
l_{0 x}^{2}=l_{0 y}^{2}=l_{0}^{2}=\frac{\hbar \xi}{\sqrt{m_{B} V_{0}}} .
$$

onde $w_{i}$ está normalizada.

- Relação entre o parâmetro de rede $d$ e healing length $\xi$ : o parâmetro de rede é definido como a distância entre os vórtices, ver Figura 15.

$$
d=2 a=\sqrt{\frac{4 \hbar}{m_{A} \Omega}} .
$$


Nota-se que $d$ decai a medida que a excitação externa $\Omega$ aumenta.

Empregando o parâmetro de Landau, Eq. (5.4), healing length e o parâmetro de rede, tem-se

$$
\frac{d^{2}}{\xi^{2}}=\frac{4 \hbar}{m_{A} \Omega} \frac{2 m_{A} g_{A} n_{A}}{\hbar^{2}}=\frac{8 g_{A} n_{A}}{\hbar \Omega} \Rightarrow \frac{d^{2}}{\xi^{2}}=16 \Gamma
$$

- Relação entre $d$ e o comprimento de oscilador $l_{0}$ : usando as equações (5.18b) e (5.19b), obtém-se

$$
\frac{d^{2}}{l_{0}^{2}}=16 \xi^{2} \Gamma \frac{\sqrt{m_{B} V_{0}}}{\hbar \xi}=\frac{16 \Gamma \sqrt{m_{B} V_{0}}}{\hbar} \frac{\hbar}{\sqrt{2 m_{A} g_{A} n_{A}}} \Rightarrow \frac{d^{2}}{4 l_{0}^{2}}=\frac{4 \Gamma}{\sqrt{2}} \sqrt{\frac{m_{B}}{m_{A}}} \sqrt{\frac{V_{0}}{g_{A} n_{A}}} .
$$

Por outro lado, utilizando a equação (5.8d) em (5.20a), consegue-se

$$
\begin{aligned}
\frac{d^{2}}{l_{0}^{2}} & =\frac{4}{\sqrt{2}} \sqrt{\frac{m_{B}}{m_{A}}} \frac{V_{0}}{g_{A} n_{A}} \Gamma=\frac{4}{\sqrt{2}} \sqrt{\frac{m_{B}}{m_{A}}}\left[\sqrt{2 \pi} \frac{m_{A}+m_{B}}{m_{A} m_{B}} \frac{a_{A B} \hbar^{2}}{\ell_{z}} \frac{m_{A} \ell_{z}}{2 \sqrt{2 \pi} \hbar^{2} a_{A}}\right]^{1 / 2} \\
& \Rightarrow \frac{d^{2}}{4 l_{0}^{2}}=2 \Gamma \sqrt{\left(1+\frac{m_{B}}{m_{A}}\right)\left(\frac{a_{A B}}{a_{A}}\right)} .
\end{aligned}
$$

- Relação entre $l_{0}$ e $\xi$ : empregando as equações (5.19b) e (5.20b), tem-se

$$
\frac{16 \Gamma \xi^{2}}{4 l_{0}^{2}}=2 \Gamma \sqrt{1+\frac{m_{B}}{m_{A}}} \sqrt{\frac{a_{A B}}{a_{A}}} \Rightarrow \frac{l_{0}^{2}}{\xi^{2}}=2 \sqrt{\frac{m_{A}}{m_{A}+m_{B}}} \sqrt{\frac{a_{A}}{a_{A B}}} .
$$

Observa-se que todas as relações apresentam dependência direta do fator de Landau $(\Gamma)$. Define-se uma escala natural de energia dos átomos da espécie $B$ aprisionados nos sítios da rede através da energia de recuo

$$
E_{R}=\frac{\hbar^{2}}{2 m_{B} d^{2}}
$$

Dessa forma, utilizando a energia de recuo e o parâmetro de rede na Eq. (5.20a), deriva-se

$$
\frac{V_{0}}{E_{R}}=8 \Gamma\left(\frac{m_{A}+m_{B}}{m_{A}}\right) \frac{a_{A B}}{a_{A}} .
$$

finalmente, substituindo na razão $V_{0} / E_{R}$ na Eq. (5.20b), consegue-se

$$
\frac{d^{2}}{4 l_{0}^{2}}=\sqrt{\frac{\Gamma}{2} \frac{V_{0}}{E_{R}}} .
$$

Definimos a função de Wannier localizada através de um perfil gausiano caracterizado pelo comprimento $l_{0}$

$$
w_{i}=w\left(x-x_{i}\right)=\left(\frac{1}{\pi l_{0}^{2}}\right)^{1 / 4} e^{-\frac{\left(x-x_{i}\right)^{2}}{2 l_{0}^{2}}}
$$




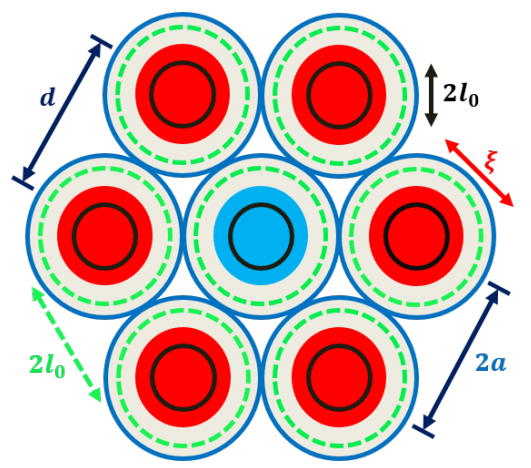

Figura 15 - Parâmetro de rede $d$, magnetic length a, healing length $\xi$ e o comprimento $l_{0}$ do perfil gaussiano da função de Wannier.

Fonte: Elaborada pelo autor.

Empregando o ansatz gaussiano é possível obter valores analíticos para os parâmetros de energia $J$ e $U$ em $\operatorname{Eq}(5.14)$.

- Tunelamento gaussiano $J_{G}$ : para o caso unidimensional (tunelamento isotrópico)

$$
\begin{aligned}
J_{G} & =-\frac{1}{\sqrt{\pi l_{0}^{2}}} \int_{-\infty}^{\infty+} e^{-\frac{\left(x-x_{i}\right)^{2}}{2 l_{0}^{2}}}\left[-\frac{\hbar^{2} \nabla^{2}}{2 m_{B}} \frac{d^{2}}{d x^{2}}+V_{0}\right] e^{-\frac{\left(x-x_{j}\right)^{2}}{2 l_{0}^{2}}} d x \\
& =\left\{\frac{\hbar^{2}}{2 m_{B} l_{0}^{2}}\left[\frac{d^{2}}{4 l_{0}^{2}}-\frac{1}{2}\right]-V_{0}\right\} e^{-\frac{d^{2}}{4 l_{0}^{2}}},
\end{aligned}
$$

Aplicando as relações (5.21) e (5.23) em (5.25a), teremos

$$
J_{G}=E_{R}\left(\frac{V_{0}}{E_{R}}\right)^{1 / 2}\left[\frac{V_{0}}{E_{R}}(2 \Gamma-1)-\sqrt{2 \Gamma}\right] e^{-2 \sqrt{\frac{\Gamma}{2} \frac{V_{0}}{E_{R}}}} .
$$

- Energia de interação $U$ : para um sistema bidimensional

$$
U=g_{B}\left\{\int_{-\infty}^{\infty+}\left[e^{-\frac{\left(x-x_{i}\right)^{2}}{2 l_{0}^{2}}} \frac{1}{\left(\pi l_{0}^{2}\right)^{1 / 4}}\right]^{4} d x\right\}^{2} \Rightarrow U=\frac{g_{B}}{2 \pi l_{0}^{2}}
$$

Empregando a expressão (5.23) em (5.26), obtemos

$$
U=\frac{8}{\sqrt{\pi}}\left(\frac{a_{B}}{l_{z}}\right) \Gamma^{1 / 2} E_{R} \sqrt{\frac{V_{0}}{E_{R}}},
$$

\subsubsection{Ponto crítico da TFQ}

A razão entre as energias $U$ e $J$ permite determinar a transição de fase do sistema, de acordo com o valor crítico obtido da solução dos lóbulos (ver seção 4.3.1). Como no 
caso anterior da rede óptica, o tunelamento $J$ será caracterizado aqui pela solução exata da menor banda de energia de Bloch, $J_{M}$. Usando as equações (4.21) e (5.27), deduzimos

$$
\frac{U}{J_{M}}=2 \sqrt{\Gamma}\left(\frac{a_{B}}{l_{z}}\right)\left(\frac{E_{R}}{V_{0}}\right)^{1 / 4} e^{2 \sqrt{V_{0} / E_{R}}}
$$

Em termos dos parâmetros da rede de vórtices, teremos

$$
\frac{U}{J_{M}}=\left(\frac{a_{B}}{l_{z}}\right)\left[2 \Gamma\left(\frac{m_{A}}{m_{A}+m_{B}}\right) \frac{a_{A}}{a_{A B}}\right]^{1 / 4} e^{4 \sqrt{2 \Gamma\left(\frac{m_{A}+m_{B}}{m_{A}}\right) \frac{a_{A B}}{a_{A}}}} .
$$

Conhecendo $U / J_{M}$ da transição de fase, pode-se determinar o comprimento de espalhamento críticos $a_{B}$ ou $a_{A B}$ para valores fixos dos demais parâmetros da Eq.(5.28b), conforme será exemplificado na seção final desse capítulo.

\subsection{Transição de fase quântica}

Conforme descrito no capítulo 4, o caminho usual para determinar o diagrama de fases é através da aproximação de mean-field para o termo cinético do Hamiltoniano de Bose-Hubbard, seguida do tratamento perturbativo para lidar com os Hamiltonianos desacoplados dos sítios (75), e para obter a equação dos lóbulos. Essa aproximação de mean-field é boa quando aplicada para dimensões superiores $(D \rightarrow \infty)$. A técnica a ser empregada para corrigir a aproximação de mean-field será a aproximação de Teoria de campos (TC). (81)

\subsubsection{Aproximação de Teoria de Campos}

Esse método consiste em reescrever o Hamiltoniano de Bose-Hubbard em termos dos parâmetros $t, t^{*}$ a serem maximizados através do método variacional, i. e., fazer $t, t^{*} \rightarrow$ 0. Nessa seção indica-se só as partes mais relevantes da técnica, a qual está detalhada na 
referência. (81)

Reescrevendo a Eq. (5.13) em termos de $t$ e $t^{*}$

$$
\hat{\mathcal{H}}_{B H} \approx-J \sum_{\langle i j\rangle} \hat{b}_{i}^{\dagger} \hat{b}_{j}+\sum_{i}\left(t^{*} \hat{b}_{i}+t \hat{b}_{i}^{\dagger}\right)+\frac{U}{2} \sum_{i} \hat{n}_{i}\left(\hat{n}_{i}-1\right)-\mu \sum_{i} \hat{n}_{i} .
$$

Expandindo a energia livre $\Xi_{o}$ associada ao sistema grande-canônico em termos de $t$ e $t^{*}$ através da série de Taylor, tem-se

$$
\Xi_{o}\left(t^{*}, t, J\right)=N_{v}\left(\Xi_{o}(J)+\sum_{p=1}^{\infty} c_{2 p}(J)|t|^{2 p}\right) \quad \text { com } \quad c_{2 p}(J)=\sum_{n=0}^{\infty}(-J)^{n} \alpha_{2 p}^{(n)},
$$

onde $N_{v}$ é o número de vórtices (lembrando que $i=1, \ldots, N_{v}$ abrange a rede toda).

Ao empregar a transformação de Legendre à energia livre $\Xi_{o}$, derivamos a energia efetiva em termos do parâmetro de ordem $\phi=\left\langle\hat{b}_{i}\right\rangle$ e $\phi^{*}=\left\langle\hat{b}_{i}^{\dagger}\right\rangle$

$$
\mathscr{E}\left(\phi, \phi^{*}, J\right)=\Xi\left(\phi, \phi^{*}, J\right) / N_{v}-\phi^{*} t-\phi t^{*}
$$

obtidos por meio de

$$
\phi=\frac{1}{N_{v}} \frac{\partial \Xi\left(\phi, \phi^{*}, J\right)}{\partial t^{*}}, \quad \phi^{*}=\frac{1}{N_{v}} \frac{\partial \Xi\left(\phi, \phi^{*}, J\right)}{\partial t} .
$$

A partir dos parâmetros de ordens $\phi\left(\phi^{*}\right)$ do sistema grande-canônico é possível derivar uma expressão para os parâmetros $t\left(t^{*}\right)$. Substituindo a equação (5.30) em (5.31b), consegue-se

$$
|t|^{2 p}=\frac{|\phi|^{2}}{c_{2}^{2 p}(J)}\left[1+\sum_{p=2}^{\infty} p \frac{c_{2 p}(J)}{c_{2}(J)} \frac{|t|^{2 p}}{|t|^{2}}\right]^{-2 p} .
$$

O parâmetro $t$ satisfaz uma equação transcendente, e sua solução depende da convergência do termo somatório. Para garantir a convergência é razoável assumir o parâmetro sendo pequeno, de forma que possa ser expandido. Aplicando o teorema binomial generalizado nessa última equação

$$
|t|^{2 p} \approx \frac{|\phi|^{2}}{c_{2}^{2 p}(J)}\left[1-2 p \sum_{p=2}^{\infty} p \frac{c_{2 p}(J)}{c_{2}(J)} \frac{|t|^{2 p}}{|t|^{2}}+\ldots\right]
$$

Dessa forma, substituindo as equações (5.30) e (5.31d) na energia efetiva, (5.31a), tem-se

$$
\mathscr{E}\left(\phi, \phi^{*}, J\right) \approx \Xi_{o}(J)+\sum_{p=1}^{\infty}(1-2 p) c_{2 p}(J) \frac{|\phi|^{2}}{c_{2}^{2 p}(J)}\left[1-2 p \sum_{p=2}^{\infty} p \frac{c_{2 p}(J)}{c_{2}(J)} \frac{|t|^{2 p}}{|t|^{2}}+\ldots\right] .
$$


Aplicando a equação de recorrência (5.31d), a energia efetiva adota a forma de uma expansão em termos do módulo quadrado do parâmetro de ordem

$$
\mathscr{E}\left(\phi, \phi^{*}, J\right) \simeq \Xi_{o}(J)-\frac{1}{c_{2}(J)}|\phi|^{2}+\frac{c_{4}(J)}{c_{2}^{4}(J)}|\phi|^{4}+\ldots
$$

Para $p=1$, o coeficiente $c_{2}(J)$, Eq. (5.30), é expandido em termos da energia de tunelamento. Neste caso considera-se relevantes os primeiros termos da expansão; devido a que essa aproximação é valida para a energia de tunelamento pequeno, afim de aplicar a teoria de perturbações para o cálculo dos coeficientes. Então

$$
\frac{1}{c_{2}(J)}=\frac{1}{\alpha_{2}^{(0)}(J)}\left[1+\sum_{n=1}^{\infty}(-J)^{n} \frac{\alpha_{2}^{(n)}}{\alpha_{2}^{(0)}(J)}\right]^{-1} \approx \frac{1}{\alpha_{2}^{(0)}}\left\{1+\frac{\alpha_{2}^{(1)}}{\alpha_{2}^{(0)}} J+\left[\left(\frac{\alpha_{2}^{(1)}}{\alpha_{2}^{(0)}}\right)^{2}-\frac{\alpha_{2}^{(2)}}{\alpha_{2}^{(0)}}\right] J^{2}+\ldots\right\} .
$$

Os parâmetros $\alpha_{2}^{(0)}, \alpha_{2}^{(1)}$ e $\alpha_{2}^{(2)}$ são obtidos usando a teoria de perturbações até quarta ordem. Seus valores são

$$
\begin{aligned}
& \alpha_{2}^{(0)}=\frac{b+1}{U(b-n)(b+1-n)}, \quad \alpha_{2}^{(1)}=z\left(\alpha_{2}^{(0)}\right)^{2}, \quad \text { com } \quad b=\mu / U \\
& \alpha_{2}^{(2)}=z^{2}\left(\alpha_{2}^{(0)}\right)^{3}+\frac{\alpha_{2}^{(1)}}{U} \frac{n(n+1)(4+3 b+2 n)\left[-3-2 n+2\left(b^{2}+b-2 b n+n^{2}\right)\right]}{(b+1)^{2}(b-n-2)(b+3-n)} .
\end{aligned}
$$

A energia $\mathscr{E}$ é equivalente à expansão de Landau, de modo que o sistema atinge a TFQ quando $1 / c_{2}(J) \rightarrow 0$. A equação dos lóbulos que corrige a aproximação de mean-field será dada por

$$
z \alpha_{2}^{(0)} J+\left[\left(z \alpha_{2}^{(0)}\right)^{2}-\frac{\alpha_{2}^{(2)}}{\alpha_{2}^{(0)}}\right] J^{2}=-1
$$

\subsubsection{Diagrama de Fases}

O gráfico da Fig. 16 representa a TFQ segundo os dois métodos analíticos descritos, considerando um preenchimento unitário da rede de vórtices. A aproximação de meanfield subestima o valor do ponto crítico da TFQ, a qual é melhor descrita através do método de Teoria de Campos. Este último corrige a aproximação de MF para sistema de baixa dimensionalidade, apresentando maior concordâmcia com os diagramas de fase numéricos. $(82,83)$ 


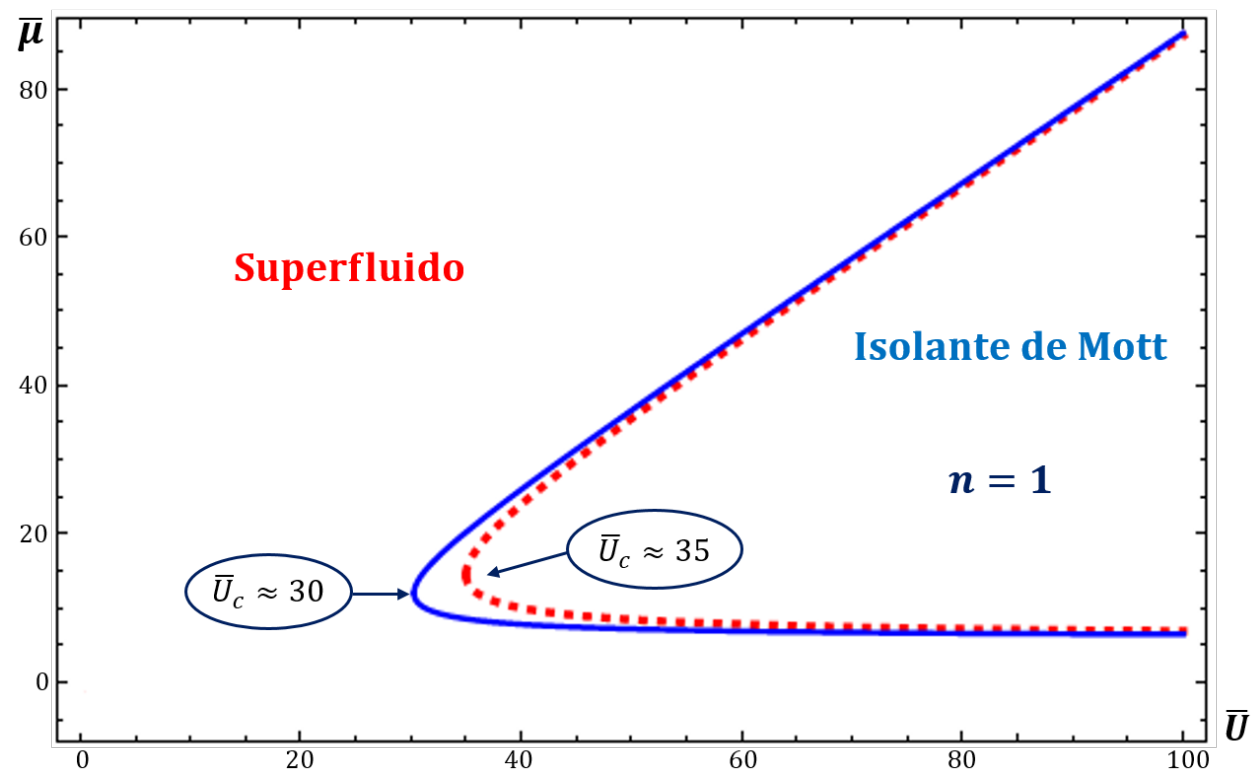

Figura 16 - Diagrama de fases para $n=1$. O lóbulo interno (vermelho tracejada) foi feita pela aproximação de mean-field, enquanto que o lóbulo exterior (azul) foi obtida pela TC através da equação (5.32) usando $z=6$.

Fonte: Elaborada pelo autor.

Segundo o gráfico, o ponto crítico da aproximação de mean-field $\left(U / J_{M}\right)_{c} \approx 35$, enquanto que o resultado da TC fornece $\left(U / J_{M}\right)_{c} \approx 30$.

Os valores experimentais estabelecidos para os comprimentos de espalhamento da onda- $s$ das espécies $A$ e $B$ e interespécies são mostrados na Tabela 1.

Tabela 1 - Valores experimentais para os espalhamentos da onda-s $a_{s}$ e massa $m$ para distintos átomos alcalinos. $a_{0}$ é o raio de Bohr

\begin{tabular}{ccccc}
\hline Espécie & $m\left(10^{-27}\right) \mathrm{kg}$ & $a_{s}\left(a_{0}\right)$ & Mistura & $a_{s}\left(a_{0}\right)$ \\
\hline${ }^{23} \mathrm{Na}$ & 38.18 & $62(84)$ & ${ }^{23} \mathrm{Na}-{ }^{87} \mathrm{Rb}$ & $73(85)$ \\
${ }^{41} \mathrm{~K}$ & 68.06 & $78(86)$ & ${ }^{41} \mathrm{~K}-{ }^{87} \mathrm{Rb}$ & $650(87)$ \\
${ }^{87} \mathrm{Rb}$ & 144.42 & $100(87)$ & ${ }^{133} \mathrm{Cs}-{ }^{87} \mathrm{Rb}$ & $206(88)$ \\
${ }^{133} \mathrm{Cs}$ & 220.78 & $280(89)$ & & \\
\hline
\end{tabular}

Fonte: Elaborada pelo autor.

A seguir empregamos o valor critico $\left(U / J_{M}\right)_{c} \approx 30$ e a equação (5.28b) para computar possíveis valores críticos do espalhamento, assumindo um certo valor para o fator de Landau dentro do regime LLL $(\Gamma<1)$. Para tal fim consideramos dois casos.

- Caso I: Nesse caso vamos determinar valores críticos do espalhamento da espécie $B$, 
mantendo fixos os espalhamentos da espécie $a_{A}$ e entre as espécies $a_{A B}$. As curvas com os valores propostos para o parâmetro de espalhamento $a_{B}$ das diferentes misturas atômicas são mostradas na Tabela 2 e Figuras 17 desenhadas em escala logarítmica.

Tabela 2 - Valores aproximados para o espalhamento $a_{B}$ com fator de Landau $\Gamma_{L L L}=0.6$ para a mistura de espécies diferentes. $l_{z}$ foi computado empregando $\omega_{z}=200 \times 2 \pi \mathrm{rad} / \mathrm{s}$.

\begin{tabular}{cccccc} 
& \multicolumn{4}{c}{ Experimentais } & Propostos \\
\hline Mistura & $l_{z}$ & $a_{A}$ & $a_{B}$ & $a_{A B}$ & $a_{B}$ \\
$A-B$ & $\left(10^{-6}\right) \mathrm{m}$ & $\left(a_{0}\right)$ & $\left(a_{0}\right)$ & $\left(a_{0}\right)$ & $\left(a_{0}\right)$ \\
\hline${ }^{23} \mathrm{Na}-{ }^{87} \mathrm{Rb}$ & 1.48 & 62 & $\mathbf{1 0 0}$ & 73 & $\mathbf{3 7 . 7}$ \\
${ }^{87} \mathrm{Rb}-{ }^{23} \mathrm{Na}$ & 0.76 & 100 & $\mathbf{6 2}$ & 73 & $\sim \mathbf{6} \times \mathbf{1 0}^{3}$ \\
${ }^{41} \mathrm{~K}-{ }^{87} \mathrm{Rb}$ & 1.11 & 78 & $\mathbf{1 0 0}$ & 650 & $\sim \mathbf{2} \times \mathbf{1 0}^{-5}$ \\
${ }^{87} \mathrm{Rb}-{ }^{41} \mathrm{~K}$ & 0.76 & 100 & $\mathbf{7 8}$ & 650 & $\mathbf{0 . 9 5}$ \\
${ }^{133} \mathrm{Cs}-{ }^{87} \mathrm{Rb}$ & 0.61 & 280 & $\mathbf{1 0 0}$ & 206 & $\sim \mathbf{3} \times \mathbf{1 0}^{3}$ \\
${ }^{87} \mathrm{Rb}-{ }^{133} \mathrm{Cs}$ & 0.76 & 100 & $\mathbf{2 8 0}$ & 206 & $\mathbf{2 8 . 3}$ \\
\hline
\end{tabular}

Fonte: Elaborada pelo autor.
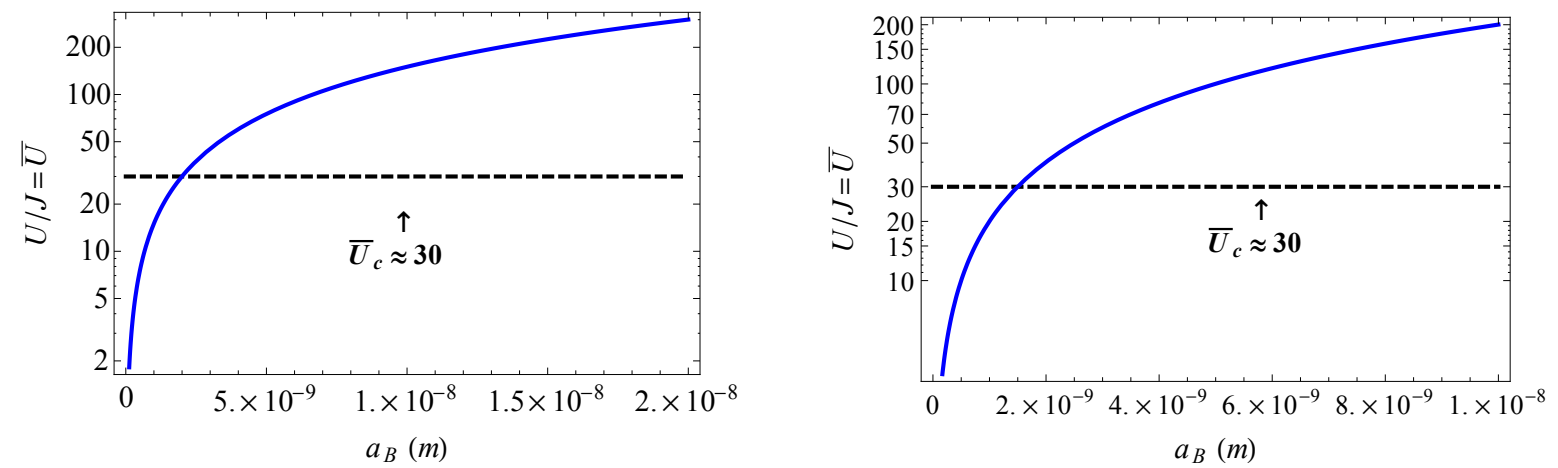

Figura 17 - Valores críticos do espalhamento $a_{B}$ para as misturas: ${ }^{23} \mathrm{Na}-{ }^{87} \mathrm{Rb}$ à esquerda e ${ }^{87} \mathrm{Rb}-{ }^{133} \mathrm{Cs}$ à direita.

Fonte: Elaborada pelo autor.

- Caso II: Nesse caso vamos determinar valores críticos do espalhamento entre as espécies $a_{A B}$, mantendo fixos os espalhamentos da espécie $a_{A}$ e espécie $a_{B}$. As curvas com os valores propostos para o parâmetro de espalhamento $a_{A B}$ das diferentes misturas atômicas são mostradas na Tabela 3 e Figuras 18, 19, as quais estão desenhadas em escala logarítmica. 
Tabela 3 - Valores aproximados para o espalhamento $a_{A B}$ com fator de Landau $\Gamma_{L L L}=0.6$ para a mistura de espécies diferentes. $l_{z}$ foi computado empregando $\omega_{z}=200 \times 2 \pi \mathrm{rad} / \mathrm{s}$.

\begin{tabular}{cccccc} 
& \multicolumn{4}{c}{ Experimentais } & Propostos \\
\hline Mistura & $l_{z}$ & $a_{A}$ & $a_{B}$ & $a_{A B}$ & $a_{A B}$ \\
$A-B$ & $\left(10^{-6}\right) \mathrm{m}$ & $\left(a_{0}\right)$ & $\left(a_{0}\right)$ & $\left(a_{0}\right)$ & $\left(a_{0}\right)$ \\
\hline${ }^{23} \mathrm{Na}-{ }^{87} \mathrm{Rb}$ & 1.48 & 62 & 100 & $\mathbf{7 3}$ & $\mathbf{5 9 . 3}$ \\
${ }^{87} \mathrm{Rb}-{ }^{23} \mathrm{Na}$ & 0.76 & 100 & 62 & $\mathbf{7 3}$ & $\mathbf{3 4 6 . 6}$ \\
${ }^{41} \mathrm{~K}-{ }^{87} \mathrm{Rb}$ & 1.11 & 78 & 100 & $\mathbf{6 5 0}$ & $\mathbf{1 0 6 . 9}$ \\
${ }^{87} \mathrm{Rb}-{ }^{41} \mathrm{~K}$ & 0.76 & 100 & 78 & $\mathbf{6 5 0}$ & $\mathbf{2 8 2 . 3}$ \\
${ }^{133} \mathrm{Cs}-{ }^{87} \mathrm{Rb}$ & 0.61 & 280 & 100 & $\mathbf{2 0 6}$ & $\mathbf{6 0 9 . 3}$ \\
${ }^{87} \mathrm{Rb}-{ }^{133} \mathrm{Cs}$ & 0.76 & 100 & 280 & $\mathbf{2 0 6}$ & $\mathbf{1 1 8}$ \\
${ }^{23} \mathrm{Na}-{ }^{41} \mathrm{~K}$ & 1.48 & 78 & 62 & - & $\mathbf{1 0 7 . 7}$ \\
${ }^{41} \mathrm{~K}-{ }^{23} \mathrm{Na}$ & 0.76 & 62 & 78 & - & $\mathbf{2 3 8 . 4}$ \\
\hline
\end{tabular}

Fonte: Elaborada pelo autor.
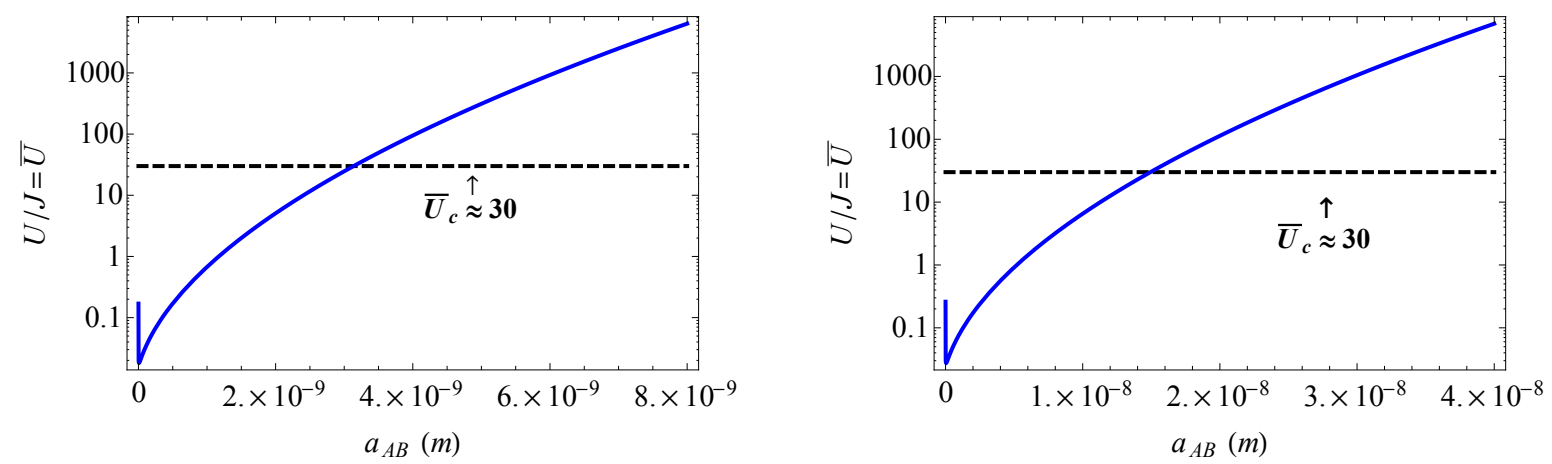

Figura 18 - Valores críticos do espalhamento $a_{A B}$ para as misturas: ${ }^{23} \mathrm{Na}-{ }^{87} \mathrm{Rb}$ à esquerda e ${ }^{87} \mathrm{Rb}-{ }^{41} \mathrm{~K}$ à direita.

Fonte: Elaborada pelo autor.
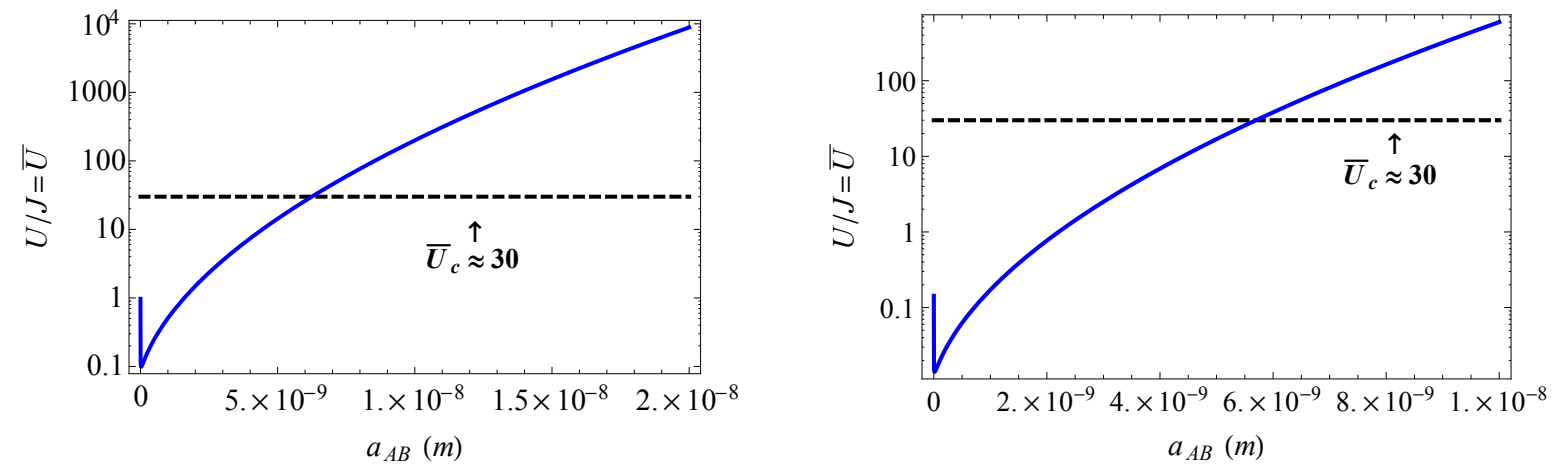

Figura 19 - Valores críticos do espalhamento $a_{A B}$ para as misturas: ${ }^{87} \mathrm{Rb}-{ }^{133} \mathrm{Cs}$ à esquerda e ${ }^{23} \mathrm{Na}-{ }^{41} \mathrm{~K}$ à direita.

Fonte: Elaborada pelo autor. 



\section{Modos vibracionais da rede de vórtices}

Neste capítulo introduz-se flutuações na rede de vórtices da espécie $A$, ou seja, os modos vibracionais da rede de vórtices, mais conhecidos como modos de Tkachenko (16,90), ver Figura 20. Esses modos fornecerão uma dinâmica própria na rede que repercutirá na sua interação com a espécie $B$. Neste caso o sistema será descrito por meio do Hamiltoniano de Bose-Hubbard estendido.
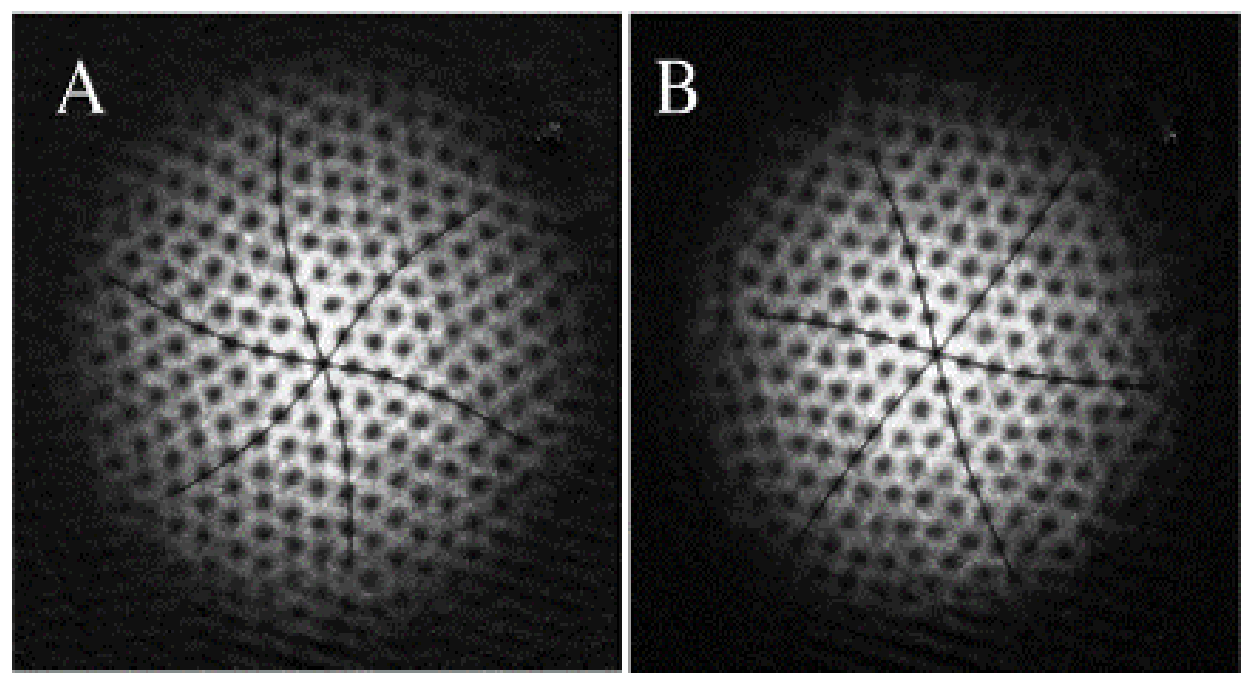

Figura 20 - Modos de Tkachenko observados no experimento do JILA no regime de TF com $\Gamma_{L L L}=0.6$.

Fonte: CODDINGTON et al. (18)

\subsection{Espécie $A$ : Transformação de Bogoliubov}

No capítulo anterior aplicamos a aproximação de MF para tratar o operador de campo da espécie $A$. Consideraremos agora as flutuações quânticas na espécie $A$, introduzidas com a redefinição do operador

$$
\hat{\psi}_{A}(\mathbf{r})=\phi_{A}(\mathbf{r})+\delta \hat{\psi}_{A}(\mathbf{r})
$$


Substituindo a equação (6.1) em (5.8a), tem-se

$$
\begin{aligned}
\hat{H}_{A}= & \int\left[\phi_{A}^{*} \hat{h}_{0} \phi_{A}+\frac{g_{A}}{2}\left|\phi_{A}\right|^{4}\right] d \mathbf{r}+\int\left[\delta \hat{\psi}_{A}^{\dagger}\left(\hat{h}_{0}+g_{A}\left|\phi_{A}\right|^{2}\right) \phi_{A}+\phi_{A}^{*}\left(\hat{h}_{0}+g_{A}\left|\phi_{A}\right|^{2}\right) \delta \hat{\psi}_{A}\right] d \mathbf{r}+ \\
& \int\left[\delta \hat{\psi}_{A}^{\dagger}\left(\hat{h}_{0}+2 g_{A}\left|\phi_{A}\right|^{2}\right) \delta \hat{\psi}_{A}+\frac{g_{A}}{2}\left(\phi_{A}^{* 2} \delta \hat{\psi}_{A} \delta \hat{\psi}_{A}+\phi_{A}^{2} \delta \hat{\psi}_{A}^{\dagger} \delta \hat{\psi}_{A}^{\dagger}\right)\right] d \mathbf{r}+ \\
& \int g_{A}\left[\phi_{A} \delta \hat{\psi}_{A}^{\dagger} \delta \hat{\psi}_{A}^{\dagger} \delta \hat{\psi}_{A}+\phi_{A}^{*} \delta \hat{\psi}_{A}^{\dagger} \delta \hat{\psi}_{A} \delta \hat{\psi}_{A}\right] d \mathbf{r}+\int \frac{g_{A}}{2}\left[\delta \hat{\psi}_{A}^{\dagger} \delta \hat{\psi}_{A}^{\dagger} \delta \hat{\psi}_{A} \delta \hat{\psi}_{A}\right] d \mathbf{r},
\end{aligned}
$$

onde $\hat{h}_{0}=(-\imath \hbar \nabla-\mathbf{A})^{2} / 2 m_{A}$.

Para fixar o número de átomos do sistema, o procedimento usual é considerar o ensemble grande-canônico, i.e. fazer $\hat{\mathcal{H}}_{A}=\hat{H}_{A}-\mu_{A} \hat{N}_{A} \operatorname{com} \hat{N}_{A}=\int \hat{\psi}_{A}^{\dagger} \hat{\psi}_{A} d \mathbf{r}$ sendo o número total de átomos e $\mu_{A}$ o potencial químico. Novamente, com a Eq. (6.1), obtém-se

$$
\hat{N}_{A}=\int\left[\left|\phi_{A}\right|^{2}+\phi_{A}^{*} \delta \hat{\psi}_{A}+\delta \hat{\psi}_{A}^{\dagger} \phi_{A}+\delta \hat{\psi}_{A}^{\dagger} \delta \hat{\psi}_{A}\right] d \mathbf{r} .
$$

Podemos separar as contribuições de $\hat{\mathcal{H}}_{A}$ de acordo com a ordem da flutuação $\delta \hat{\psi}_{A}$

$$
\hat{\mathcal{H}}_{A}=\mathcal{H}_{0}+\hat{\mathcal{H}}_{1}+\hat{\mathcal{H}}_{2}+\hat{\mathcal{H}}_{3}+\hat{\mathcal{H}}_{4}
$$

onde

$$
\begin{aligned}
& \mathcal{H}_{0}=\int\left[\phi_{A}^{*}\left(\hat{h}_{0}-\mu\right) \phi_{A}+\frac{g_{A}}{2}\left|\phi_{A}\right|^{4}\right] d \mathbf{r} \\
& \hat{\mathcal{H}}_{1}=\int\left[\delta \hat{\psi}_{A}^{\dagger}\left(\hat{h}_{0}+g_{A}\left|\phi_{A}\right|^{2}-\mu\right) \phi_{A}+\phi_{A}^{*}\left(\hat{h}_{0}+g_{A}\left|\phi_{A}\right|^{2}-\mu\right) \delta \hat{\psi}_{A}\right] d \mathbf{r} \\
& \hat{\mathcal{H}}_{2}=\int\left[\delta \hat{\psi}_{A}^{\dagger}\left(\hat{h}_{0}+2 g_{A}\left|\phi_{A}\right|^{2}-\mu\right) \delta \hat{\psi}_{A}+\frac{g_{A}}{2}\left(\phi_{A}^{* 2} \delta \hat{\psi}_{A} \delta \hat{\psi}_{A}+\phi_{A}^{2} \delta \hat{\psi}_{A}^{\dagger} \delta \hat{\psi}_{A}^{\dagger}\right)\right] d \mathbf{r} \\
& \hat{\mathcal{H}}_{3}=\int g_{A}\left[\phi_{A} \delta \hat{\psi}_{A}^{\dagger} \delta \hat{\psi}_{A}^{\dagger} \delta \hat{\psi}_{A}+\phi_{A}^{*} \delta \hat{\psi}_{A}^{\dagger} \delta \hat{\psi}_{A} \delta \hat{\psi}_{A}\right] d \mathbf{r} \\
& \hat{\mathcal{H}}_{4}=\int \frac{g_{A}}{2}\left[\delta \hat{\psi}_{A}^{\dagger} \delta \hat{\psi}_{A}^{\dagger} \delta \hat{\psi}_{A} \delta \hat{\psi}_{A}\right] d \mathbf{r},
\end{aligned}
$$

apresentando ordens $0,1,2,3$ e 4, respectivamente.

Os operadores $\delta \hat{\psi}_{A}$ e $\delta \hat{\psi}_{A}^{\dagger}$ satisfazem a relações de comutação:

$$
\left[\delta \hat{\psi}_{A}(\mathbf{r}), \delta \hat{\Psi}_{A}^{\dagger}\left(\mathbf{r}^{\prime}\right)\right]=\delta\left(\mathbf{r}-\mathbf{r}^{\prime}\right) \quad \text { e } \quad\left[\delta \hat{\psi}_{A}(\mathbf{r}), \delta \hat{\psi}_{A}\left(\mathbf{r}^{\prime}\right)\right]=\left[\delta \hat{\psi}_{A}^{\dagger}(\mathbf{r}), \delta \hat{\psi}_{A}^{\dagger}\left(\mathbf{r}^{\prime}\right)\right]=0
$$

Considerando a dependência temporal do operador de campo $\hat{\psi}_{A}(\mathbf{r}) \rightarrow \hat{\Psi}_{A}(\mathbf{r}, t)$ e $\phi_{A}(\mathbf{r}) \rightarrow$ $\Phi_{A}(\mathbf{r}, t)$, através da equação de Heisemberg Eq. (2.10), deriva-se a equação dinâmica

$$
\begin{aligned}
i \hbar \frac{\partial}{\partial t} \delta \hat{\Psi}_{A}= & g_{A}\left|\Phi_{A}\right|^{2} \delta \hat{\Psi}_{A}+g_{A} \Phi_{A}^{2} \delta \hat{\Psi}_{A}^{\dagger}+g_{A} \Phi_{A} \delta \hat{\Psi}_{A}^{\dagger} \delta \hat{\Psi}_{A^{+}} \\
& \left(\hat{h}_{0}+g_{A}\left|\Phi_{A}\right|^{2}\right) \delta \hat{\Psi}_{A}+g_{A} \Phi_{A}^{*} \delta \hat{\Psi}_{A} \delta \hat{\Psi}_{A}+g_{A} \Phi_{A} \delta \hat{\Psi}_{A}^{\dagger} \delta \hat{\Psi}_{A}+g_{A} \delta \hat{\Psi}_{A}^{\dagger} \delta \hat{\Psi}_{A} \delta \hat{\Psi}_{A}
\end{aligned}
$$


onde foi empregada a equação dinâmica da função macroscópica $\Phi_{A}$ obtida por meio da aproximação de MF, Eq. (2.13).

Linearizando a Eq. (6.6), isto é, considerando apenas os termos até primeira ordem na flutuação, finalmente obtém-se

$$
i \hbar \frac{\partial}{\partial t} \delta \hat{\Psi}_{A}(\mathbf{r}, t)=\left[\hat{h}_{0}+2 g_{A}\left|\Phi_{A}(\mathbf{r}, t)\right|^{2}\right] \delta \hat{\Psi}_{A}(\mathbf{r}, t)+g_{A} \Phi_{A}^{2}(\mathbf{r}, t) \delta \hat{\Psi}_{A}^{\dagger}(\mathbf{r}, t)
$$

\subsubsection{Expansão dos modos vibracionais}

Para caracterizar as flutuações da rede de vórtices da espécie $A$, expandimos o operador da flutução em termos dos modos de Bogoliubov (79)

$$
\delta \hat{\psi}_{A}(\mathbf{r})=\frac{1}{\sqrt{S}} \sum_{\mathbf{q}}\left(u_{\mathbf{q}}(\mathbf{r}) \hat{a}_{\mathbf{q}}-v_{\mathbf{q}}(\mathbf{r}) \hat{a}_{\mathbf{q}}^{\dagger}\right)
$$

$S$ : Representa a área da cela primitiva do espaço real.

$u_{\mathbf{q}}(\mathbf{r}), v_{\mathbf{q}}(\mathbf{r})$ : Funções escalares a serem determinadas.

$\hat{a}_{\mathbf{q}}, \hat{a}_{\mathbf{q}}^{\dagger}$ : Operadores de criação e destruição dos modos de Bogoliubov,

q: Pseudo-momentum restrito à 1ZB.

Os operadores $\hat{a}_{\mathbf{q}}, \hat{a}_{\mathbf{q}}^{\dagger}$ satisfazem as relações de comutação

$$
\left[\hat{a}_{\mathbf{q}}, \hat{a}_{\mathbf{q}^{\prime}}^{\dagger}\right]=\delta_{\mathbf{q q}^{\prime}} ; \quad\left[\hat{a}_{\mathbf{q}}^{\dagger}, \hat{a}_{\mathbf{q}^{\prime}}^{\dagger}\right]=\left[\hat{a}_{\mathbf{q}}, \hat{a}_{\mathbf{q}^{\prime}}\right]=0
$$

Utilizando as relações de comutação dos operadores de campo originais e as dos operadores de Bogoliubov, podemos extrair a seguinte relação entre os coeficientes da Eq. (6.8):

$$
\begin{aligned}
& \delta\left(\mathbf{r}-\mathbf{r}^{\prime}\right)= \delta \hat{\psi}_{A}(\mathbf{r}) \delta \hat{\psi}_{A}^{\dagger}\left(\mathbf{r}^{\prime}\right)-\delta \hat{\psi}_{A}^{\dagger}\left(\mathbf{r}^{\prime}\right) \delta \hat{\psi}_{A}(\mathbf{r})=\frac{1}{S} \sum_{\mathbf{q}^{\prime}}\left\{u_{\mathbf{q}}(\mathbf{r}) u_{\mathbf{q}^{\prime}}^{*}\left(\mathbf{r}^{\prime}\right)\left[\hat{a}_{\mathbf{q}}, \hat{a}_{\mathbf{q}^{\prime}}^{\dagger}\right]+\right. \\
&\left.u_{\mathbf{q}}(\mathbf{r}) v_{\mathbf{q}^{\prime}}^{*}\left(\mathbf{r}^{\prime}\right)\left[\hat{a}_{\mathbf{q}}, \hat{a}_{\mathbf{q}^{\prime}}\right]+v_{\mathbf{q}}(\mathbf{r}) u_{\mathbf{q}^{\prime}}^{*}\left(\mathbf{r}^{\prime}\right)\left[\hat{a}_{\mathbf{q}}^{\dagger}, \hat{a}_{\mathbf{q}^{\prime}}^{\dagger}\right]+v_{\mathbf{q}}(\mathbf{r}) v_{\mathbf{q}^{\prime}}^{*}\left(\mathbf{r}^{\prime}\right)\left[\hat{a}_{\mathbf{q}}^{\dagger}, \hat{a}_{\mathbf{q}^{\prime}}\right]\right\} \\
& \underbrace{=}_{(6.9)} \frac{1}{S} \sum_{\mathbf{q q}^{\prime}}\left[u_{\mathbf{q}}(\mathbf{r}) u_{\mathbf{q}^{\prime}}^{*}\left(\mathbf{r}^{\prime}\right)-v_{\mathbf{q}}(\mathbf{r}) v_{\mathbf{q}^{\prime}}^{*}\left(\mathbf{r}^{\prime}\right)\right] \delta_{\mathbf{q q}^{\prime}}
\end{aligned}
$$

$\therefore$

$$
\frac{1}{S} \sum_{\mathbf{q}}\left[u_{\mathbf{q}}(\mathbf{r}) u_{\mathbf{q}}^{*}\left(\mathbf{r}^{\prime}\right)-v_{\mathbf{q}}(\mathbf{r}) v_{\mathbf{q}}^{*}\left(\mathbf{r}^{\prime}\right)\right]=\delta\left(\mathbf{r}-\mathbf{r}^{\prime}\right) .
$$


De forma análoga

$$
\frac{1}{S} \sum_{\mathbf{q}}\left[u_{\mathbf{q}}(\mathbf{r}) v_{\mathbf{q}}^{*}\left(\mathbf{r}^{\prime}\right)-v_{\mathbf{q}}^{*}(\mathbf{r}) u_{\mathbf{q}}\left(\mathbf{r}^{\prime}\right)\right]=\frac{1}{S} \sum_{\mathbf{q}}\left[u_{\mathbf{q}}^{*}(\mathbf{r}) v_{\mathbf{q}}\left(\mathbf{r}^{\prime}\right)-v_{\mathbf{q}}(\mathbf{r}) u_{\mathbf{q}}^{*}\left(\mathbf{r}^{\prime}\right)\right]=0 .
$$

Consideramos a dependência temporal do operador flutuação:

$$
\delta \hat{\Psi}_{A}^{\dagger}(\mathbf{r}, t)=\frac{1}{\sqrt{S}} \sum_{\mathbf{q}}\left(u_{\mathbf{q}}(\mathbf{r}) \hat{a}_{\mathbf{q}} e^{-i\left(\mu+\epsilon_{q}\right) t / \hbar}-v_{\mathbf{q}}(\mathbf{r}) \hat{a}_{\mathbf{q}}^{\dagger} e^{-i\left(\mu-\epsilon_{q}\right) t / \hbar}\right)
$$

$\operatorname{com} \epsilon_{q}$ sendo a energia associada à evolução temporal do operador de Bogoliubov.

Substituindo a equação (6.11) em (6.7) e igualando os coeficientes das exponenciais, obtêm-se o sistema

$$
\left\{\begin{array}{l}
\left(\hat{h}_{0}+2 g_{A}\left|\phi_{A}\right|^{2}-\mu\right) u_{\mathbf{q}}-g_{A} \phi_{A}^{2} v_{\mathbf{q}}^{*}=\epsilon_{q} u_{\mathbf{q}} \\
\left(\hat{h}_{0}+2 g_{A}\left|\phi_{A}\right|^{2}-\mu\right) v_{\mathbf{q}}-g_{A} \phi_{A}^{2} u_{\mathbf{q}}^{*}=-\epsilon_{q} v_{\mathbf{q}} .
\end{array}\right.
$$

As Eqs. (6.12) são conhecidas como equações de Bogoliubov - De Gennes, as quais foram resolvidas analiticamente por Matvenko. (78)

\subsubsection{Hamiltoniano de Bogoliubov}

O Hamiltoniano (6.3) será aproximado até segunda ordem da flutuação, em concordância com a transformação de Bogoliubov, de forma que $\hat{\mathcal{H}}_{3} \sim \hat{\mathcal{H}}_{4} \sim 0$. Verificamos também que o termo linear $\hat{\mathcal{H}}_{1}$ é nulo ao considerar a validade da GPE, Eq. (2.15). Teremos então

$$
\hat{\mathcal{H}}_{A} \sim \mathcal{H}_{0}+\hat{\mathcal{H}}_{2}
$$

O próximo passo consiste em diagonalizar o Hamiltoniano (6.13a). Para isso empregaremos o sistema de equações Bogoliubov-De Gennes e as Eqs. (6.10a)-(6.10b) para obter relações entre os coeficientes $u_{\mathbf{q}}$ e $v_{\mathbf{q}}$.

Na sequência detalhamos algumas passagens para obtenção do $\hat{\mathcal{H}}_{A}$ diagonal, partindo da aproximação em Eq. (6.13a). 
- Analisando $\hat{\mathcal{H}}_{2}$ : Com equação (6.8), obtêm-se

$$
\begin{aligned}
\hat{\mathcal{H}}_{2}=\int\{ & \frac{1}{S} \sum_{\mathbf{q}_{\mathbf{q}^{\prime}}}\left[u_{\mathbf{q}}^{*} \hat{D} u_{\mathbf{q}^{\prime}}-\frac{g_{A}}{2}\left(\phi_{A}^{2} u_{\mathbf{q}}^{*} v_{\mathbf{q}^{\prime}}^{*}+\phi_{A}^{* 2} v_{\mathbf{q}} u_{\mathbf{q}^{\prime}}\right)\right] \hat{a}_{\mathbf{q}^{\prime}}^{\dagger} \hat{a}_{\mathbf{q}^{\prime}} \\
& \left.+\left[v_{\mathbf{q}}^{*} \hat{D} v_{\mathbf{q}^{\prime}}-\frac{g_{A}}{2}\left(\phi_{A}^{2} v_{\mathbf{q}}^{*} u_{\mathbf{q}^{\prime}}^{*}+\phi_{A}^{* 2} u_{\mathbf{q}} v_{\mathbf{q}^{\prime}}\right)\right] \hat{a}_{\mathbf{q}} \hat{a}_{\mathbf{q}^{\prime}}^{\dagger}\right\} \\
+\int\{ & \frac{1}{S} \sum_{\mathbf{q q}^{\prime}}\left[-u_{\mathbf{q}}^{*} \hat{D} v_{\mathbf{q}^{\prime}}+\frac{g_{A}}{2}\left(\phi_{A}^{2} u_{\mathbf{q}}^{*} u_{\mathbf{q}^{\prime}}^{*}+\phi_{A}^{* 2} v_{\mathbf{q}} v_{\mathbf{q}^{\prime}}\right)\right] \hat{a}_{\mathbf{q}^{\prime}} \hat{a}_{\mathbf{q}^{\prime}}^{\dagger} \\
+ & {\left.\left[-v_{\mathbf{q}}^{*} \hat{D} u_{\mathbf{q}^{\prime}}+\frac{g_{A}}{2}\left(\phi_{A}^{2} v_{\mathbf{q}}^{*} v_{\mathbf{q}^{\prime}}^{*}+\phi_{A}^{* 2} u_{\mathbf{q}} u_{\mathbf{q}^{\prime}}\right)\right] \hat{a}_{\mathbf{q}} \hat{a}_{\mathbf{q}^{\prime}}\right\} }
\end{aligned}
$$

onde $\hat{D}=\hat{h}_{0}+2 g_{A}\left|\phi_{A}\right|^{2}-\mu$.

Nota-se que apenas as duas primeiras linhas da equação (6.13b) são diagonais nos operadores dos modos. A forma de diagonalizar o Hamiltoniano é zerar os coeficientes dos termos não diagonais da Eq.(6.13b):

$$
\int \frac{1}{S} \sum_{\mathbf{q q}^{\prime}}\left(\begin{array}{c}
-u_{\mathbf{q}}^{*} \hat{D} v_{\mathbf{q}^{\prime}}+\frac{g_{A}}{2}\left(\phi_{A}^{2} u_{\mathbf{q}}^{*} u_{\mathbf{q}^{\prime}}^{*}+\phi_{A}^{* 2} v_{\mathbf{q}} v_{\mathbf{q}^{\prime}}\right) \\
-v_{\mathbf{q}}^{*} \hat{D} u_{\mathbf{q}^{\prime}}+\frac{g_{A}}{2}\left(\phi_{A}^{2} v_{\mathbf{q}}^{*} v_{\mathbf{q}^{\prime}}^{*}+\phi_{A}^{* 2} u_{\mathbf{q}} u_{\mathbf{q}^{\prime}}\right)
\end{array}\right) d \mathbf{r}=\left(\begin{array}{l}
0 \\
0
\end{array}\right)
$$

De forma que

$$
\int \frac{1}{S} \sum_{\mathbf{q}^{\prime}}\left[v_{\mathbf{q}}^{*} \hat{D} u_{\mathbf{q}^{\prime}}-u_{\mathbf{q}} \hat{D}^{\dagger} v_{\mathbf{q}^{\prime}}^{*}\right] d \mathbf{r}=0 \quad \Rightarrow \quad \int v_{\mathbf{q}}^{*}\left[\hat{D} u_{\mathbf{q}^{\prime}}\right] d \mathbf{r}=\int\left[\hat{D}^{\dagger} v_{\mathbf{q}^{\prime}}^{*}\right] u_{\mathbf{q}} d \mathbf{r} .
$$

Portanto, mostra-se que o operador $\hat{D}$ é hermitiano para qualquer função $u_{\mathbf{q}}, v_{\mathbf{q}}$, assim

$$
\hat{D}^{\dagger}=\hat{D}
$$

Por outro lado, ao multiplicar $u_{\mathbf{q}}$, $v_{\mathbf{q}}^{\star}$ e seus complexos conjugados nas equações de Bogoliubov - De Gennes (6.12), obtêm-se

$$
\left\{\begin{array}{l}
-\hat{D} v_{\mathbf{q}} v_{\mathbf{q}}^{*}+g_{A} \phi_{A}^{2} u_{\mathbf{q}}^{*} v_{\mathbf{q}}^{*}=\epsilon_{q} v_{\mathbf{q}} v_{\mathbf{q}}^{*} \\
\hat{D}^{\dagger} u_{\mathbf{q}}^{*} u_{\mathbf{q}}-g_{A} \phi_{A}^{* 2} v_{\mathbf{q}} u_{\mathbf{q}}=\epsilon_{q} u_{\mathbf{q}}^{*} u_{\mathbf{q}} \underbrace{\Rightarrow}_{(-)}\left(\hat{D}+\epsilon_{q}\right) v_{\mathbf{q}} v_{\mathbf{q}}^{*}-\left(\hat{D}^{\dagger}-\epsilon_{q}\right) u_{\mathbf{q}}^{*} u_{\mathbf{q}}+g_{A}\left[\phi_{A}^{* 2} u_{\mathbf{q}} v_{\mathbf{q}}-\phi_{A}^{2} u_{\mathbf{q}}^{*} v_{\mathbf{q}}^{*}\right]=0
\end{array}\right.
$$

$$
\left\{\begin{array}{l}
\hat{D} u_{\mathbf{q}} u_{\mathbf{q}}^{*}-g_{A} \phi_{A}^{2} v_{\mathbf{q}}^{*} u_{\mathbf{q}}^{*}=\epsilon_{q} u_{\mathbf{q}} u_{\mathbf{q}}^{*} \\
-\hat{D}^{\dagger} v_{\mathbf{q}}^{*} v_{\mathbf{q}}+g_{A} \phi_{A}^{* 2} u_{\mathbf{q}} v_{\mathbf{q}}=\epsilon_{q} v_{\mathbf{q}}^{*} v_{\mathbf{q}} \\
\underbrace{\Rightarrow}_{(+)}\left(\hat{D}-\epsilon_{q}\right) u_{\mathbf{q}} u_{\mathbf{q}}^{*}-\left(\hat{D}^{\dagger}+\epsilon_{q}\right) v_{\mathbf{q}}^{*} v_{\mathbf{q}}+g_{A}\left[\phi_{A}^{* 2} u_{\mathbf{q}} v_{\mathbf{q}}-\phi_{A}^{2} u_{\mathbf{q}}^{*} v_{\mathbf{q}}^{*}\right]=0
\end{array}\right.
$$

Usando a hermiticidade (6.13d), e somando as equações (6.14a) e (6.14b), deduz-se

$$
\phi_{A}^{* 2} u_{\mathbf{q}} v_{\mathbf{q}}=\phi_{A}^{2} u_{\mathbf{q}}^{*} v_{\mathbf{q}}^{*}
$$


Substituindo a relação dada pela Eq. (6.14c) nos termos diagonais da Eq. (6.13b):

$$
\begin{aligned}
\hat{\mathcal{H}}_{2} & =\int\left\{\frac{1}{S} \sum_{\mathbf{q} \mathbf{q}^{\prime}}\left[\epsilon_{\vec{q}^{\prime}} u_{\mathbf{q}^{\prime}}^{*} u_{\mathbf{q}^{\prime}}\right] \hat{a}_{\mathbf{q}^{\prime}}^{\dagger} \hat{a}_{\mathbf{q}^{\prime}}\right\} d \mathbf{r}-\int\left\{\frac{1}{S} \sum_{\mathbf{q q}^{\prime}}\left[\epsilon_{\vec{q}^{\prime}} v_{\mathbf{q}^{\prime}}^{*} v_{\mathbf{q}}\right] \hat{a}_{\mathbf{q}^{\prime}} \hat{a}_{\mathbf{q}}^{\dagger}\right\} d \mathbf{r} \\
& \underbrace{=}_{(6.9)} \int \frac{1}{S} \sum_{\mathbf{q}_{\mathbf{q}^{\prime}}}\left[\epsilon_{\vec{q}^{\prime}}\left(u_{\mathbf{q}}^{*} u_{\mathbf{q}^{\prime}}-v_{\mathbf{q}^{\prime}}^{*} v_{\mathbf{q}}\right) \hat{a}_{\mathbf{q}^{\prime}}^{\dagger} \hat{a}_{\mathbf{q}^{\prime}}-\epsilon_{\vec{q}^{\prime}} v_{\mathbf{q}^{\prime}}^{*} v_{\mathbf{q}^{\prime}} \delta_{\mathbf{q}^{\prime} \mathbf{q}}\right] d \mathbf{r} \\
& =\frac{1}{S} \sum_{\mathbf{q q}_{\mathbf{q}^{\prime}}} \epsilon_{\vec{q}^{\prime}} \hat{a}_{\mathbf{q}}^{\dagger} \hat{a}_{\mathbf{q}^{\prime}} \int\left(u_{\mathbf{q}}^{*} u_{\mathbf{q}^{\prime}}-v_{\mathbf{q}^{\prime}}^{*} v_{\mathbf{q}}\right) d \mathbf{r}-\int \frac{1}{S} \sum_{\mathbf{q q}_{\mathbf{q}^{\prime}}} \epsilon_{\vec{q}^{\prime}} v_{\mathbf{q}^{\prime}}^{*} v_{\mathbf{q}} \delta_{\mathbf{q}^{\prime} \mathbf{q}} d \mathbf{r} \\
& =\sum_{\mathbf{q} \mathbf{q}^{\prime}} \epsilon_{\vec{q}^{\prime}} \hat{a}_{\mathbf{q}}^{\dagger} \hat{a}_{\mathbf{q}^{\prime}} \delta_{\mathbf{q}^{\prime} \mathbf{q}}-\frac{1}{S} \sum_{\mathbf{q}} \epsilon_{\mathbf{q}} \int\left|v_{\mathbf{q}}\right|^{2} d \mathbf{r} .
\end{aligned}
$$

Derivamos então o $\hat{\mathcal{H}}_{2}$ diagonalizado na aproximação de Bogoliubov

$$
\hat{\mathcal{H}}_{2}^{B O G}=\sum_{\mathbf{q}} \epsilon_{\mathbf{q}} \hat{a}_{\mathbf{q}}^{\dagger} \hat{a}_{\mathbf{q}}-\frac{1}{S} \sum_{\mathbf{q}} \epsilon_{\mathbf{q}} \int\left|v_{\mathbf{q}}\right|^{2} d \mathbf{r}
$$

O segundo termo será considerado como uma correção no Hamiltoniano de $\mathrm{MF} \mathcal{H}_{0}$.

\subsection{Interação entre as espécies: $A-B$}

Partindo novamente da expansão em Eq. (6.3), consideraremos agora os termos que advém do Hamiltoniano de interação $\hat{H}_{A B}$. Conforme visto anteriormente, o termo de ordem zero fornece potencial de rede $\hat{H}_{\text {rede }}^{A B}$ associado com o Hamiltoniano de mean-field.

Por outro lado, o termo de interação de primeira ordem fornecerá o Hamiltoniano da interação entre a espécie $B$ com os modos de Bogoliubov da rede, o qual denominaremos $\hat{H}_{\text {modo-rede. }}^{A B}$ Quanto ao termo de segunda ordem na flutuação, é esperado que forneça um deslocamento da energia dos modos de Bogoliubov. Como esse deslocamento é proporcional à densidade dos atomos da espécie $B$, o qual considera-se muito pequena, será factível desprezarmos a última correção.

Dada essas observações todas, teremos finalmente

$$
\begin{aligned}
& \hat{H}^{A B} \approx \hat{H}_{\text {rede }}^{A B}+\hat{H}_{\text {modo-rede }}^{A B} \\
& \hat{H}^{A B} \approx g_{A B} \int\left[n_{A}\left|\varphi_{A}\right|^{2}\right] \hat{\psi}_{B}^{\dagger} \hat{\psi}_{B} d \mathbf{r}+g_{A B} \sqrt{n_{A}} \int\left[\varphi_{A}^{*} \delta \hat{\psi}_{A}+\varphi_{A} \delta \hat{\psi}_{A}^{\dagger}\right] \hat{\psi}_{B}^{\dagger} \hat{\psi}_{B} d \mathbf{r} .
\end{aligned}
$$




\section{Hamiltoniano associado ao modo-rede}

Aplicando a transformação de Bogoliubov, Eq. (6.8), no segundo termo do Hamiltoniano entre as espécies, Eq. (6.16), tem-se

$$
\begin{aligned}
\hat{H}_{\text {modo-rede }}^{A B} & =g_{A B} \sqrt{n_{A}} \int\left[\varphi_{A}^{*}\left(\frac{1}{\sqrt{S}} \sum_{\mathbf{q}}\left[u_{\mathbf{q}} \hat{a}_{\mathbf{q}}-v_{\mathbf{q}} \hat{a}_{\mathbf{q}}^{\dagger}\right]\right)+\varphi_{A}\left(\frac{1}{\sqrt{S}} \sum_{\mathbf{q}}\left[u_{\mathbf{q}}^{*} \hat{a}_{\mathbf{q}}^{\dagger}-v_{\mathbf{q}}^{*} \hat{a}_{\mathbf{q}}\right]\right)\right] \hat{\psi}_{B}^{\dagger} \hat{\psi}_{B} d \mathbf{r} \\
& =g_{A B} \frac{\sqrt{n_{A}}}{\sqrt{S}} \int \sum_{\mathbf{q}}\left[\left(\varphi_{A}^{*} u_{\mathbf{q}}-\varphi_{A} v_{\mathbf{q}}^{*}\right) \hat{a}_{\mathbf{q}}+\left(\varphi_{A} u_{\mathbf{q}}^{*}-\varphi_{A}^{*} v_{\mathbf{q}}\right) \hat{a}_{\mathbf{q}}^{\dagger}\right] \hat{\psi}_{B}^{\dagger} \hat{\psi}_{B} d \mathbf{r} .
\end{aligned}
$$

Utilizando expansão do operador de campo da espécie $B$ em termos das funções de Wannier, equação (5.10), na Eq. (6.17a), consegue-se

$$
\hat{H}_{\text {modo-rede }}^{A B}=g_{A B} \frac{\sqrt{n_{A}}}{\sqrt{S}} \sum_{i j} \sum_{\mathbf{q}}\left[\Omega_{\mathbf{q}}^{i j} \hat{a}_{\mathbf{q}}+\bar{\Omega}_{\mathbf{q}}^{i j} \hat{a}_{\mathbf{q}}^{\dagger}\right] \hat{b}_{i}^{\dagger} \hat{b}_{j},
$$

onde

$$
\begin{aligned}
& \Omega_{\mathbf{q}}^{i j}=\int\left[\varphi_{A}^{*} u_{\mathbf{q}}-\varphi_{A} v_{\mathbf{q}}^{*}\right] w_{i}^{*} w_{j} d \mathbf{r} \\
& \bar{\Omega}_{\mathbf{q}}^{i j}=\int\left[\varphi_{A} u_{\mathbf{q}}^{*}-\varphi_{A}^{*} v_{\mathbf{q}}\right] w_{i}^{*} w_{j} d \mathbf{r} .
\end{aligned}
$$

\subsection{Hamiltoniano total do sistema estendido}

Este Hamiltoniano é dado por

$$
\begin{aligned}
\hat{\mathcal{H}} & =\hat{\mathcal{H}}_{A}^{B O G}+\hat{\mathcal{H}}_{B}+\hat{H}_{\text {rede }}^{A B}+\hat{H}_{\text {modo-rede }}^{A B} \\
& =\hat{\mathcal{H}}_{A}^{B O G}+\hat{\mathcal{H}}_{B}^{B H}+\hat{H}_{\text {modo-rede }}^{A B} .
\end{aligned}
$$

Substituindo os Hamiltonianos aproximados com respeito à transformação de Bogoliubov, Eqs. (6.13a) e (6.17b), e o Hamiltoniano de Bose-Hubbard da espécie B, Eq. (5.13), derivase o $\hat{\mathcal{H}}$ em presença das flutuações

$$
\hat{\mathcal{H}} \approx \sum_{\mathbf{q}} \epsilon_{\mathbf{q}} \hat{a}_{\mathbf{q}}^{\dagger} \hat{a}_{\mathbf{q}}-J \sum_{\langle i j\rangle} \hat{b}_{i}^{\dagger} \hat{b}_{j}-\mu \sum_{i} \hat{n}_{i}+\frac{U}{2} \sum_{i} \hat{n}_{i}\left(\hat{n}_{i}-1\right)+g_{A B} \frac{\sqrt{n_{A}}}{\sqrt{S}} \sum_{i j} \sum_{\mathbf{q}}\left[\Omega_{\mathbf{q}}^{i j} \hat{a}_{\mathbf{q}}+\bar{\Omega}_{\mathbf{q}}^{i j} \hat{a}_{\mathbf{q}}^{\dagger}\right] \hat{b}_{i}^{\dagger} \hat{b}_{j}
$$

onde a contribuição de $\hat{\mathcal{H}}_{0}$ foi omitida, dado que pode ser considerada como um deslocamento da energia total. Os parâmetros de energia $\mathbf{J}, \mathbf{U}$ e $\mu$ estão dados pelas relações 
(5.14a), (5.14b) e (5.14c) respectivamente.

O passo seguinte consiste em determinar o Hamiltoniano que descreva a dinâmica da espécie $B$ na presença dos modos vibracionais $\left(\hat{a}_{\mathbf{q}}, \hat{a}_{\mathbf{q}}^{\dagger}\right)$.

\subsubsection{Transformação unitária}

Na sequência aplicaremos uma transformação unitária no Hamiltoniano do sistema a fim de obtermos o modelo de Bose-Hubbard efetivo para espécie $B$, isto é, um Hamiltoniano "vestido". Trata-se da chamada transformação de polaron (91), a qual nos permitirá embutir os efeitos do espalhamento com os modos de Bogoliubov, último termo da Eq. (6.19), como correções nos parâmetros do modelo BH efetivo dos átomos $B$.

Considera-se a transfomação

$$
\tilde{\mathrm{H}}=e^{\hat{\zeta}} \hat{\mathcal{H}} e^{-\hat{\zeta}}=\hat{\mathcal{H}}+[\hat{\zeta}, \hat{\mathcal{H}}]+\frac{1}{2 !}[\hat{\zeta},[\hat{\zeta}, \hat{\mathcal{H}}]]+\ldots
$$

Com $\hat{\zeta}$ sendo um operador definido como

$$
\hat{\zeta}=\frac{1}{\sqrt{S}} \sum_{\mathbf{q} i}\left[e^{i \mathbf{q} \cdot \mathbf{R}_{i}} \alpha_{\mathbf{q} i}^{*} \hat{a}_{\mathbf{q}}^{\dagger}-e^{-i \mathbf{q} \cdot \mathbf{R}_{i}} \alpha_{\mathbf{q} i} \hat{a}_{\mathbf{q}}\right] \hat{n}_{i}
$$

onde $\alpha_{\mathbf{q} i}$ é um parâmetro a ser determinado.

Para determinar o Hamiltoniano transformado em Eq. (6.20a), começamos aplicando a transformação sobre o operador da impureza e dos modos da rede:

- Operadores $\tilde{\hat{b}}_{i}$ e $\tilde{\hat{b}}_{i}^{\dagger}$ : Usando a relação em (6.20a), tem-se

$$
\tilde{\hat{b}}_{i}=\hat{b}_{i} e^{\hat{Y}_{i}} \quad \text { e } \quad \tilde{\hat{b}}_{i}^{\dagger}=\hat{b}_{i}^{\dagger} e^{-\hat{Y}_{i}}
$$

com

$$
\hat{Y}_{i}=-\frac{1}{\sqrt{S}} \sum_{\mathbf{q}}\left[e^{i \mathbf{q} \cdot \mathbf{R}_{i}} \alpha_{\mathbf{q} i}^{*} \hat{a}_{\mathbf{q}}^{\dagger}-e^{-i \mathbf{q} \cdot \mathbf{R}_{i}} \alpha_{\mathbf{q} i} \hat{a}_{\mathbf{q}}\right]
$$

- Operadores $\tilde{\hat{a}}_{\mathbf{q}}$ e $\tilde{\hat{a}}_{\mathbf{q}}^{\dagger}$ : Nesse caso, obtemos

$$
\tilde{\hat{a}}_{\mathbf{q}}=\hat{a}_{\mathbf{q}}-\frac{1}{\sqrt{S}} \sum_{i} e^{i \mathbf{q} \cdot \mathbf{R}_{i}} \alpha_{\mathbf{q} i}^{*} \hat{n}_{i} \quad \text { e } \quad \tilde{\hat{a}}_{\mathbf{q}}^{\dagger}=\hat{a}_{\mathbf{q}}^{\dagger}-\frac{1}{\sqrt{S}} \sum_{i} e^{-i \mathbf{q} \cdot \mathbf{R}_{i}} \alpha_{\mathbf{q} i} \hat{n}_{i}
$$




\subsubsection{Hamiltoniano de Bose-Hubbard estendido: $\alpha$ particular}

Para obter o modelo de Bose-Hubbard estendido para espécie $B$, aplicaremos sobre o Hamiltoniano transformado em Eq. (6.20a) o traço ou média sobre os graus de liberdade correspondente aos modos da rede. Representamos os modos através do estado $|f\rangle=\prod_{\mathbf{q}}\left|N_{\mathbf{q}}\right\rangle$, onde o produtório abrange todos os estados de ocupação da $1 Z B$, que correspondem ao operador número $\hat{N}_{\mathbf{q}}=\hat{a}_{\mathbf{q}}^{\dagger} \hat{a}_{\mathbf{q}}$. O Hamiltoniano de Bose-Hubbard "vestido" para a espécie $B$ será obtido de (ver Apêndices A e B para detalhes)

$$
\mathbf{H}=\langle f|\tilde{\mathrm{H}}| f\rangle .
$$

O próximo passo consiste em determinar o valor do parâmetro $\alpha_{i \mathbf{q}}$ (transformação de polaron). Uma solução razoável para $\alpha_{i \mathbf{q}}$ será aquela que diagonaliza o Hamiltoniando em Eq. (6.22), com a eliminação do termo de interação $H_{\text {modo-rede }}^{A B}$ Trata-se de uma aproximação adiabática, em que desprezamos efeitos de retardamento na interação entre $B$ e os modos da rede. Esse limite é razoável no regime de baixas energias para os átomos $B$, de forma que existe um ajuste instantâneo da configuração dos modos espalhados perante à ação de B. De acordo com a Eq. (A.1), obteremos as relacões

$$
\Omega_{\mathbf{q}}^{i j}=\frac{\epsilon_{\mathbf{q}}}{g_{A B} \sqrt{n_{A}}} e^{-i \mathbf{q} \cdot \mathbf{R}_{i}} \alpha_{i \mathbf{q}} \delta_{i j} \quad \text { e } \quad \bar{\Omega}_{\mathbf{q}}^{i j}=\frac{\epsilon_{\mathbf{q}}}{g_{A B} \sqrt{n_{A}}} e^{i \mathbf{q} \cdot \mathbf{R}_{i}} \alpha_{i \mathbf{q}}^{*} \delta_{i j}
$$

Para $i=j$ teremos

$$
\alpha_{i \mathbf{q}}=\frac{g_{A B} \sqrt{n_{A}}}{\epsilon_{\mathbf{q}}} e^{i \mathbf{q} \cdot \mathbf{R}_{i}} \Omega_{i \mathbf{q}} \quad \text { e } \quad \alpha_{i \mathbf{q}}^{*}=\frac{g_{A B} \sqrt{n_{A}}}{\epsilon_{\mathbf{q}}} e^{i \mathbf{q} \cdot \mathbf{R}_{i}} \Omega_{i \mathbf{q}}^{*}
$$

Derivamos também as seguintes equações

$$
\begin{aligned}
& \alpha_{j \mathbf{q}}^{*} \alpha_{i \mathbf{q}}=\frac{n_{A} g_{A B}^{2}}{\epsilon_{\mathbf{q}}^{2}} \Omega_{j \mathbf{q}}^{*} \Omega_{i \mathbf{q}} e^{-i \mathbf{q} \cdot \mathbf{d}} \Rightarrow\left|\alpha_{i \mathbf{q}}\right|^{2}=\frac{n_{A} g_{A B}^{2}}{\epsilon_{\mathbf{q}}^{2}}\left|\Omega_{i \mathbf{q}}\right|^{2} \\
& \Omega_{i \mathbf{q}} e^{i \mathbf{q} \cdot \mathbf{R}_{i}} \alpha_{i \mathbf{q}}^{*}+\Omega_{i \mathbf{q}}^{*} e^{-i \mathbf{q} \cdot \mathbf{R}_{i}} \alpha_{i \mathbf{q}}=\frac{2 \sqrt{n_{A}} g_{A B}}{\epsilon_{\mathbf{q}}}\left|\Omega_{i \mathbf{q}}\right|^{2} \\
& \Omega_{j \mathbf{q}} e^{i \mathbf{q} \cdot \mathbf{R}_{i}} \alpha_{i \mathbf{q}}^{*}+\Omega_{j \mathbf{q}}^{*} e^{-i \mathbf{q} \cdot \mathbf{R}_{i}} \alpha_{i \mathbf{q}}=\frac{\sqrt{n_{A}} g_{A B}}{\epsilon_{\mathbf{q}}}\left[\Omega_{j \mathbf{q}}^{*} \Omega_{i \mathbf{q}}+\Omega_{i \mathbf{q}}^{*} \Omega_{j \mathbf{q}}\right] .
\end{aligned}
$$

Nesta última parte consideramos que interação entre vizinhos é isotrópica e restrita aos primeiros vizinhos somente. Além disso, empregamos fato de que $\Omega_{\mathbf{q}}^{i j}, \bar{\Omega}_{\mathbf{q}}^{i j} \sim \delta_{i j}$, equação 
(6.23a), para eliminar termos que contenham a forma $\sum_{\langle i j\rangle}\left(\Omega_{\mathbf{q}}^{i j}, \bar{\Omega}_{\mathbf{q}}^{i j}\right)=0$, dentro do Hamiltoniano total, Eq. (A.1).

Substituindo as relações (6.24a)-(6.24c) nas equações (A.3) e (A.4a)-(A.4d), deriva-se o Hamiltoniano de Bose-Hubbard estendido associado à espécie $B$

$$
\mathbf{H} \approx-\tilde{\mathbf{J}} \sum_{\langle i j\rangle} \hat{b}_{i}^{\dagger} \hat{b}_{j}+\frac{1}{2} \tilde{\mathbf{U}} \sum_{i} \hat{n}_{i}\left(\hat{n}_{i}-1\right)-\tilde{\mu}_{B} \sum_{i} \hat{n}_{i}+\frac{1}{2} \sum_{\langle i j\rangle} \mathbf{V}_{i j} \hat{n}_{i} \hat{n}_{j}
$$

com

$$
\begin{aligned}
\tilde{\mathbf{J}} & =J \exp \left[-\frac{g_{A B}^{2} n_{A}}{S} \sum_{\mathbf{q}} \frac{\left|\Omega_{i, \mathbf{q}}\right|^{2}}{\epsilon_{\mathbf{q}}^{2}}\left|\left(1-e^{-i \mathbf{q} \cdot \mathbf{d}}\right)\right|^{2}\right] \\
\tilde{\mathbf{U}} & =U-2 \frac{g_{A B}^{2} n_{A}}{S} \sum_{\mathbf{q}} \frac{\left|\Omega_{i \mathbf{q}}\right|^{2}}{\epsilon_{\mathbf{q}}} \\
\tilde{\mu} & =\mu+\frac{g_{A B}^{2} n_{A}}{S} \sum_{\mathbf{q}} \frac{\left|\Omega_{i \mathbf{q}}\right|^{2}}{\epsilon_{\mathbf{q}}} \\
\mathbf{V}_{i j} & =-2 \frac{g_{A B}^{2} n_{A}}{S} \sum_{\mathbf{q}} \frac{\Omega_{i, \mathbf{q}}^{*} \Omega_{j, \mathbf{q}}}{\epsilon_{\mathbf{q}}} .
\end{aligned}
$$

A presença dos modos vibracionais na rede de vórtices altera o comportamento dos parâmetros de energia $J, U$ e $\mu$ determinados para o caso da rede "estática". O tunelamento induzido pelos modos decai exponencialmente em comparação com o caso "estático", isto é, os átomos apresentam menor energia para tunelar entre sítios vizinhos. A energia de interação é deslocada do seu valor na rede "estática". Iremos assumir que Ũ permanece maior que zero, isto é, a correção $g_{A B}^{2}$ se manterá pequena frente ao valor original do U. O potencial químico é incrementado de um valor positivo. Comparando a Eq. (6.25) com o modelo Bose-Hubbard dado pela Eq. (5.13), verificamos que o efeito principal da inclusão dos modos na rede foi a indução de uma interação de longo alcance atrativa entre os átomos. O alcance desse potencial será considerado posteriormente com a aproximação de primeiros vizinhos, com base na sua forma analítica para pequenos valores de $\mathbf{q}$. 


\subsubsection{Transição de fase quântica: Aproximação de MF}

Nessa seção determinaremos a equação que permitirá obtermos o diagrama de fases do sistema por meio da aproximação perturbativa de MF. O primeiro passo consiste em determinar a energia do sistema empregando os operadores de criação e destruição, $\hat{b}_{i}$ e $\hat{b}_{i}^{\dagger}$, da espécie $B$. Temos um potencial de longo alcance isotrópico, ou seja, com igual magnitude qualquer que seja o par de sítios vizinhos da rede, $\mathbf{V}_{i j}=\mathbf{V}$. O Hamiltoniano de Bose-Hubbard estendido da espécie $B$ ficará

$$
\mathbf{H} \approx-\tilde{\mathbf{J}} \sum_{\langle i j\rangle} \hat{b}_{i}^{\dagger} \hat{b}_{j}+\frac{1}{2} \tilde{\mathbf{U}} \sum_{i} \hat{n}_{i}\left(\hat{n}_{i}-1\right)-\tilde{\mu}_{B} \sum_{i} \hat{n}_{i}+\frac{1}{2} \mathbf{V} \sum_{\langle i j\rangle} \hat{n}_{i} \hat{n}_{j}
$$

Para diagonalizar o Hamiltoniano na Eq. (6.27), vamos aplicar a aproximação de MF tanto para os operadores de criação $\left(\hat{b}_{i}=\phi_{i}+\delta \hat{b}_{i}\right.$, sendo $\phi_{i}=\left\langle\hat{b}_{i}\right\rangle$ o parâmetro de ordem) como para o operador número $\left(\hat{n}_{i}=\left\langle\hat{n}_{i}\right\rangle+\delta \hat{n}_{i}\right.$, sendo $n_{i}=\left\langle\hat{n}_{i}\right\rangle$ a densidade de sítios na rede). Além disso, o potencial de longo alcance será reescrito como $\mathbf{V}=-\tilde{\mathbf{V}} \operatorname{com} \tilde{\mathbf{V}}>0$. O Hamiltoniano de MF de um sitio será dado por (para detalhes ver Apêndice C)

$$
\mathbf{H}^{M F}=\sum_{i} \mathbf{H}_{i}^{M F}=\sum_{i} \mathbf{H}_{i}^{p e r}+\sum_{i} \mathbf{H}_{i}^{0}
$$

sendo $\mathbf{H}_{i}^{\text {per }}=-\tilde{\mathbf{J}}\left[\hat{b}_{i}^{\dagger} \tilde{\phi}_{i}+\hat{b}_{i} \tilde{\phi}_{i}^{*}\right]$ o Hamiltoniano de perturbação e $\mathbf{H}_{i}^{0}=\frac{1}{2} \tilde{\mathbf{U}} \hat{n}_{i}\left(\hat{n}_{i}-1\right)-\tilde{\mu} \hat{n}_{i}-$ $\frac{1}{2} \tilde{\mathbf{V}}\left[2 \tilde{n}_{i} \hat{n}_{i}-n_{i} \tilde{n}_{i}\right]+\tilde{\mathbf{J}} \phi_{i}^{*} \tilde{\phi}_{i}$ o Hamiltoniano do estado fundamental, onde $\phi_{i}=\sum_{\langle i\rangle_{j}}\left\langle\hat{b}_{i}\right\rangle$, $\tilde{n}_{i}=\sum_{\langle i\rangle_{j}} n_{j}$ e $\langle i\rangle_{j}$ representa os primeiros vizinhos do sítio $i$.

Na sequência vamos determinar a equação que permita obtermos o diagrama de fases do sistema; para isso, vamos calcular a correção na energia do estado fundamental do sistema até segunda ordem na perturbação $\mathbf{H}_{i}^{\text {per }}$

$$
E_{n} \approx E_{n_{i}}^{0}+\left\langle n_{i}\left|\mathbf{H}_{i}^{\text {per }}\right| n_{i}\right\rangle+\sum_{m_{i} \neq n_{i}} \frac{\left|\left\langle m_{i}\left|\mathbf{H}_{i}^{p e r}\right| n_{i}\right\rangle\right|^{2}}{\left(E_{n_{i}}^{0}-E_{m_{i}}^{0}\right)} .
$$

Empregando a equação de estado, Eq. (C.6d), para determinar as energias $E_{n_{i}}^{0}, E_{n_{i}+1}^{0}$ e $E_{n_{i}-1}^{0}$, assim como os elementos de matriz $\left\langle m_{i}\left|\mathbf{H}_{i}^{\text {per }}\right| n_{i}\right\rangle$, Eq.(C.8b), derivamos a equação dos lóbulos em termos do parâmetro de ordem (para detalhes ver o Apêndice C)

$$
\phi_{i}=\tilde{\phi}_{i} \tilde{\mathbf{J}}\left\{\frac{n_{i}+1}{\tilde{\mathbf{U}} n_{i}-\tilde{\mu}-\tilde{\mathbf{V}} \tilde{n}_{i}}+\frac{n_{i}}{-\tilde{\mathbf{U}}\left(n_{i}-1\right)+\tilde{\mu}+\tilde{\mathbf{V}} \tilde{n}_{i}}\right\} .
$$


Esta última relação também foi obtida por Lewenstein $(54,92)$ nos seus estudos do gás de bóson dipolar ultrafrio em rede óptica. Para determinar o limite entre as fases MI e SF, vamos considerar que o parâmetro de ordem seja real e espacialmente isotrópico $\phi_{i} \rightarrow \phi$ na equação dos lóbulos, (6.30), de forma que

$$
\tilde{\phi}_{i}=z \phi_{i} \quad \text { e } \quad \tilde{n}_{i}=z n_{i}
$$

onde $z$ é o número de primeiros vizinhos. Aplicando essa aproximação, deduzimos

$$
1=z \tilde{\mathbf{J}}\left\{\frac{n+1}{\tilde{\mathbf{U}} n-\tilde{\mu}-z \tilde{\mathbf{V}} n}+\frac{n}{-\tilde{\mathbf{U}}(n-1)+\tilde{\mu}+z \tilde{\mathbf{V}} n}\right\},
$$

com o domínio de validade estabelecido através de

$$
(n-1) \tilde{\mathbf{U}}-z n \tilde{\mathbf{V}} \leqslant \tilde{\mu} \leqslant n \tilde{\mathbf{U}}-z n \tilde{\mathbf{V}} .
$$

De agora em diante adotaremos a notação simplificada $n_{i}=n$, dado o desacoplamento dos sítios da rede perante a aproximação feita sobre a uniformidade do parâmetro de ordem.

Para obter o ponto crítico da TFQ, relacionaremos o potencial de interação $\tilde{\mathbf{U}}$ com o potencial de longo alcançe $\tilde{\mathbf{V}}$ através da equação

$$
z \tilde{\mathbf{V}}=a \tilde{\mathbf{U}}, \quad \operatorname{com} \quad a \geqslant 0(\in \mathbb{R})
$$

Usando as transformações $X=\tilde{\mathbf{U}} / z \tilde{\mathbf{J}}$ e $y=\tilde{\mu} / z \tilde{\mathbf{J}}$, reescrevemos a equação dos lóbulos como

$$
1 \approx\left\{\frac{n+1}{-y+n X+a X n}+\frac{n}{y-(n-1) X-a X n}\right\}
$$

Com $a=0$ retornamos ao resulatdo do Bose-Hubbard padrão, Eq. (4.37).

Computando essa última equação, e após igualar as soluções superior e inferior para os lóbulos, obtemos o ponto crítico da TFQ

$$
X_{c}=\left(\frac{\tilde{\mathbf{U}}}{z \tilde{\mathbf{J}}}\right)_{c}=(2 n+1) \pm \sqrt{(2 n+1)^{2}-1}=\left(\frac{U}{J}\right)_{c}^{M F}
$$

Para graficar o diagrama de fases, vamos reescrever a Eq. (6.33) para o preenchimento unitário $(n=1)$; chamando $b=\tilde{\mu} / \tilde{\mathbf{U}}$, obtemos

$$
\frac{z \tilde{\mathbf{J}}}{\tilde{\mathbf{U}}}=\left[\frac{2}{-b+1-a}+\frac{1}{b-a}\right]^{-1} \text {. }
$$




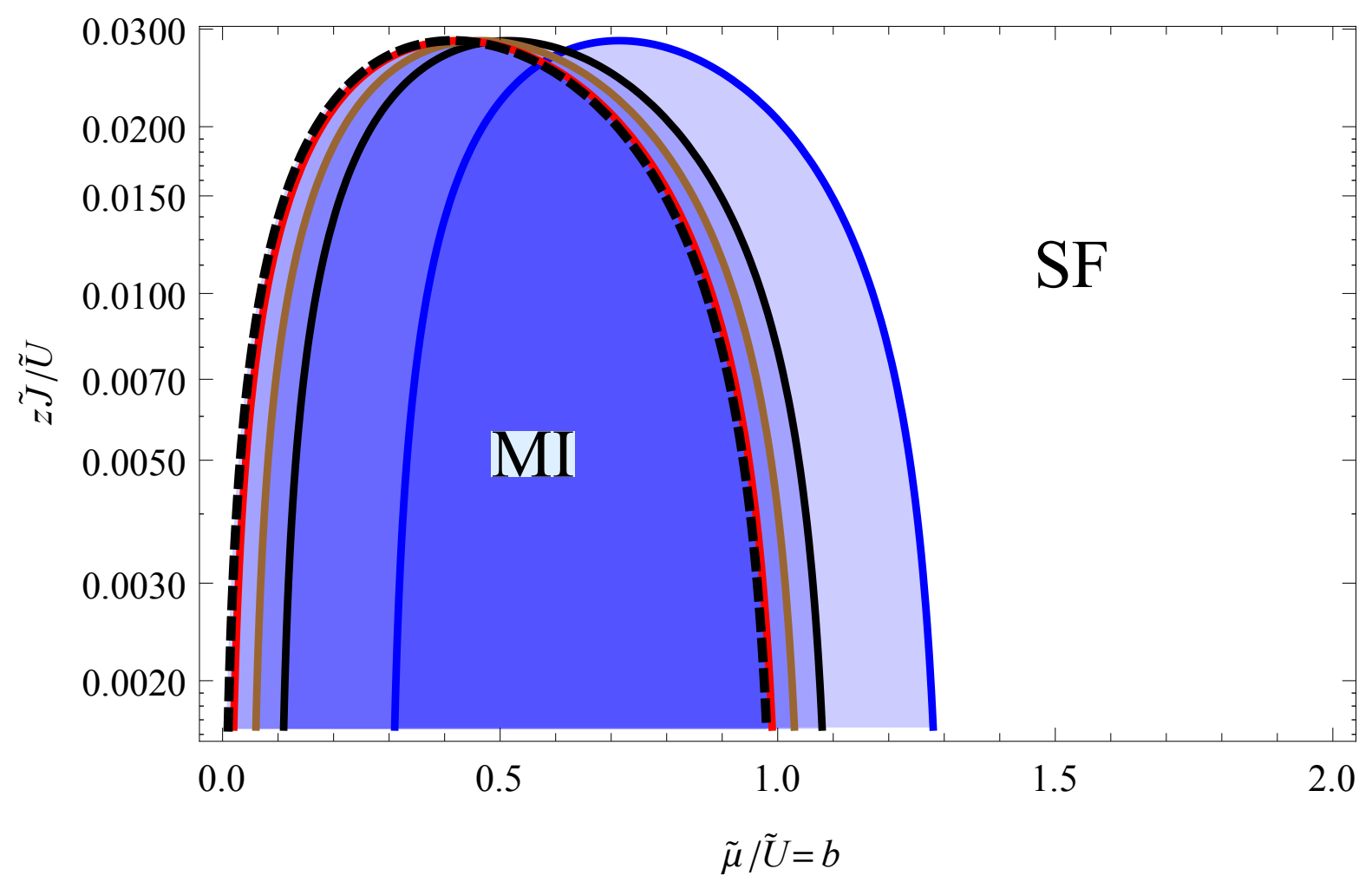

Figura 21 - Diagrama de fases para o sistema estendido, caso $\tilde{\mathbf{V}} \ll \tilde{\mathbf{U}}$. Lóbulo com preenchimento unitário: linha tracejada representando cálculo MF com $a=0$; em vermelho $a=0.01$, em marrom $a=0.05$, em preto, $a=0.1 \mathrm{em}$ azul $a=0.3$.

Fonte: Elaborada pelo autor.

Na Figura 21, mostra-se os diagramas de fases para certos valores do parâmetro $a$.

Verificamos que dentro da aproximação de mean-field, o potencial de longo alcance apenas desloca a posição dos lóbulos do diagrama anterior da rede estática. Apesar do valor do ponto crítico ser equivalente ao caso da rede "estática", o efeito do potencial de longo alcance traz mudanças sobre a expressão dos parâmetos $\tilde{\mathbf{U}}$ e $\tilde{\mathbf{J}}$, e consequentemente afetará os valores dos comprimentos de espalhamentos relativos à transição. 



\section{Conclusões}

Neste trabalho desenvolvemos um modelo de Bose-Hubbard para átomos ultrafrios interagindo com um sistema BEC da rede de vórtices. Estudamos nosso sistema considerando o potencial da rede de vórtices com e sem a presença de flutuações quânticas, ou seja, dos modos de Tkachenko da rede.

Caracterizamos a espécie $B$ através do modelo de Bose-Hubbard, considerando o regime de tight-binding com a ocupação da menor banda de energia de Bloch; associamos esse limite com o regime LLL, assumindo $\Gamma_{L L L}<1$, o qual garante a estabilidade da rede de vórtices. Tomando em conta essas considerações, determinamos o ponto crítico da transição de fase quântica em termos dos parâmetros fundamentais dos átomos: massa e comprimento de espalhamento. Dada a viabilidade experimental de modular os comprimentos de espalhamento das espécies atômicas, selecionamos determinadas misturas de gases bosônicos comumente realizadas nos laboratórios para graficar o respectivo intervalo de parâmetros para obtenção da transição de fase.

Com a inclusão das flutuações, o sistema pôde ser descrito através do modelo de Bose-Hubbard estendido, com novos termos tais como o potencial de longo alcance $\tilde{\mathbf{V}}_{\mathbf{i j}}$, Eq. (A.4d), e os potenciais de "atenuação" $\tilde{\mathbf{P}}_{\mathbf{i j}}$ e $\tilde{\mathbf{Q}}_{\mathbf{i j}}$, Eqs. (A.4e) e (A.4f), em forma geral. Nesses estudos assumimos o parâmentro de ordem e a ocupação dos sítios sendo uniformes. A solução analítica do Hamiltoniano da aproximação de MF forneceu o ponto crítico da TFQ com os novos parâmetros de energia $\tilde{\mathbf{J}}$ e $\tilde{\mathbf{U}}$.

Além da descrição particular feita até agora, o nosso sistema pode ser estudado em forma mais geral. O próximo passo do trabalho consistirá em predizer novas fases quânticas para os átomos aprisionados, além da transição de fases superfluida-isolante de Mott analisada até aqui. Determinaremos como a competição entre as energias correspondentes às interações de curto e longo-alcance com o termo cinético contribuirão para o estabelecimento de novas configurações para o estado fundamental do sistema na rede de vórtices. O fato de estarmos tratando de uma rede triangular com interação de longo 
alcance atrativa tornam nosso modelo bastante inovador dentro da comunidade de átomos frios, onde costumam ser analisadas interações de longo alcance de caráter repulsivo, em redes sem possibilidade de frustração.

Para determinar o diagrama de fases mais geral do nosso sistema, assumiremos apenas a aproximação primeiros vizinhos, tanto para o termo cinético como para interação mediada. Mapearemos o nosso Hamiltoniano Bose-Hubbard estendido no modelo de "spin1/2 XXZ ferromagnétic". (93,94) A solução analítica para resolver o diagrama resultará também da aproximação de mean-field aplicada posteriormente sobre o modelo de spins. Uma outra possibilidade seria a de determinar o diagrama numericamente, empregando a técnica de Monte-Carlo quântico para o sistema da rede em duas dimensões. 


\section{Referências}

1 MYATT, C. J. et al. Production of two overlapping Bose-Einstein condensates by sympathetic cooling. Physical Review Letters, v. 78, n. 4, p. 586-589, 1997.

2 MATTHEWS, M. R. et al. Watching a superfluid untwist itself: recurrence of Rabi oscillations in a Bose-Einstein condensate. Physical Review Letters, v. 83, n. 17, p. 3358-3361, 1999.

3 MATTHEWS, M. R. et al. Watching a superfluid untwist itself: recurrence of Rabi oscillations in a Bose-Einstein condensate. Physical Review Letters, v. 83, n. 17, p. 2498-2501, 1999.

4 SCHWEIKHARD, V. et al. Vortex-lattice dynamics in rotating spinor Bose-Einstein condensates. Physical Review Letters, v. 93, n. 21, p. 210403, 2004.

5 MOdugno, G. et al. Two atomic species superfluid. Physical Review Letters, v. 89, n. 19, p. 190404, 2002.

6 CATANI, J. et al. Degenerate Bose-Bose mixture in a three-dimensional optical lattice. Physical Review A, v. 77, n. 1, p. 011603, 2008.

7 CATANI, J. et al. Entropy exchange in a mixture of ultracold atoms. Physical Review Letters, v. 103, n. 14, p. 140401, 2009.

8 THALHAMMER, G. et al. Double species Bose-Einstein condensates with tunable interespecies interactions. Physical Review Letters, v. 100, n. 21, p. 210402, 2008.

9 PAPP, S. B.; PINO, J. M.; WIEMAN, C. E. Tunable miscibility in a dual-species Bose-Einstein condensate. Physical Review Letters, v. 101, n. 4, p. 040402, 2008.

10 JAKSCH, D.; ZOLLER, P. The cold atom Hubbard toolbox. Annals of Physics, v. 315, n. 1 , p. $52-79,2004$.

11 BLOCH, I.; DALIBARD, J.; ZWERGER, W. Many-body physics with ultracold gases. Reviews of Modern Physics, v. 80, n. 3, p. 885-964, 2008.

12 FISHER, M. P. A. et al. Boson localization and the superfluid-insulator transition. Physical Review B, v. 40, n. 1, p. 546-570, 1989. 
13 JAKSCH, D. et al. Cold bosonic atoms in optical lattices. Physical Review Letters, v. 81, n. 15, p. 3108-3111, 1998.

14 ABO-SHAEER, J. R.; RAMAN, C.; KETTERLE, W. Formation and decay of vortex lattices in Bose-Einstein condensates at finite temperatures. Physical Review Letters, v. 88, n. 7, p. 070409, 2002.

15 ABO-SHAEER, J. R. et al. Observation of vortex lattices in Bose-Einstein condensates. Science, v. 292, n. 5516, p. 476-479, 2001.

16 TKACHENKO, V. K. Stability of vortex lattices. Journal of Experimental and Theoretical Physics, v. 23, n. 6, p. 1049-1056, 1966.

17 BAYM, G. Tkachenko modes of vortex Lattices in rapidly rotating Bose-Einstein condensates. Physical Review Letters, v. 91, n. 11, p. 110402, 2003.

18 CODDINGTON, I. et al. Observation of Tkachenko oscillations in rapidly rotating Bose-Einstein condensates. Physical Review Letters, v. 91, n. 10, p. 100402, 2003.

19 LIN, Y.-J. et al. Synthetic magnetic fields for ultracold neutral atoms. Nature, v. 462, n. 7273 , p. 628-632, 2009.

20 PITAEVSKII, L.; STRINGARI, S. Bose-Einstein condensation. Oxford: Oxford University Press, 2003. 392 p. ISBN 978-0-19-850719-2.

21 PETHICK, C. J.; SMITH, H. Bose-Einstein condensation in dilute gases. 2nd ed. Cambridge: Cambridge University Press, 2008. 569 p. ISBN 978-0-521-84651-6.

22 BOSE, S. N. Plancks gesetz und lichtquantenhypothese. Zeitschrift für Physik, v. 26, n. 1, p. 178-181, 1924.

23 EINSTEIN, A. Quantentheorie de einatomigen ideal gases-zweite. Sitzungsberichte der Preussischen Akademie der Wissenschaften, v. 1, p. 3-14, 1925.

24 KAPITZA, P. L. Viscosity of liquid helium below the $\lambda$-point. Nature, v. 141, n. 3558 , p. $74-74,1938$.

25 ALLEN J. F., M. A. D. Flow of liquid helium II. Nature, v. 141, n. 3558, p. 75-75, 1938. 
26 LONDON, F. The $\lambda$-phenomenon of liquid helium and the Bose-Einstein degeneracy. Nature, v. 141, n. 3571, p. 643-644, 1938.

27 LANDAU, L. The theory of superfuidity of helium II. Physical Review, v. 60, n. 4, p. 356-358, 1941.

28 BOGOLIUBOV, N. N. On the theory of superfluidity. Journal Physics USSR, v. 11, n. 1, p. 23-32, 1947.

29 GROSS, E. P. Structure of a quantized vortex in boson systems. Nuovo Cimento, v. 20, n. 3, p. 454-457, 1961.

30 PITAEVSKII, L. P. Vortex line in an imperfect Bose gas. Journal of Experimental and Theoretical Physics, v. 13, n. 2, p. 451-454, 1961.

31 COHEN-TANNOUDJI, C. N. Manipulating atoms with photons. Reviews of Modern Physics, v. 70, n. 3, p. 707-719, 1998.

32 PHILLIPS, W. D. Laser cooling and trapping of neutral atoms. Reviews of Modern Physics, v. 70, n. 3, p. 721-742, 1998.

33 CHU, S. The manipulating of neutral particles. Reviews of Modern Physics, v. 70, n. 3, p. 685-706, 1998.

34 ANDERSON, M. H. et al. Observation of Bose-Einstein condensation in a dilute atomic vapor. Science, v. 269, n. 5221, p. 198-201, 1995.

35 DAVIS, K. B. et al. Bose-Einstein condensation in a gas of sodium atoms. Physical Review Letters, v. 75, n. 22, p. 3969-3973, 1995.

36 BRADLEY, C. C. et al. Evidence of Bose-Einstein condensation in an atomic gas with attractive interactions. Physical Review Letters, v. 75, n. 9, p. 1687-1690, 1995.

37 KETTERLE, W.; PRITCHARD, D. Atomic quantum gases, group of Wolfgang Ketterle and Dave Pritchard. Massachusetts Institute of Technology, USA. Disponível em: < http://cua.mit.edu/ketterle_group/Projects_1995/Three_peaks/3peaks.JPG>. Acesso em: 10 fev. 2016. 
38 CORNELL, E. Group of Eric Cornell. University of Colorado at Boulder, USA. Disponível em: <http://www.colorado.edu/physics/2000/bec/three_peaks.html>. Acesso em: 10 fev. 2016.

39 ALMEIDA, Z. B. Condensado de Bose-Einstein: el quinto estado de agregación de la materia. Disponível em: < http://condensadoboseeinstein.blogspot.com.br/2013/ 11/condensado-de-bose-einstein.html>. Acesso em: 10 fev. 2016.

40 LEGGETT, A. L. Bose-Einstein condensation in the alkali gases: some fundamental concepts. Reviews of Modern Physics, v. 73, n. 2, p. 307-356, 2001.

41 DALFOVO, F. et al. Theory of Bose-Einstein condensation in trapped gases. Reviews of Modern Physics, v. 71, n. 3, p. 463-512, 1999.

42 FETTER, A. L. Rotating trapped Bose-Einstein condensates. Reviews of Modern Physics, v. 81, n. 2, p. 647-691, 2009.

43 MADISON, K. W. et al. Vortex formation in a stirred Bose-Einstein condensate. Physical Review Letters, v. 84, n. 5, p. 806-809, 2000.

44 ANDERSON, B. P. et al. Vortex precession in Bose-Einstein condensates: observations with filled and empty Cores. Physical Review Letters, v. 85, n. 14, p. 2857-2860, 2000.

45 HALJAN, P. C. et al. Driving Bose-Einstein-condensate vorticity with a rotating normal cloud. Physical Review Letters, v. 87, n. 21, p. 210403, 2002.

46 LEANHARDT, A. E. et al. Imprinting vortices in a Bose-Einstein condensate using topological phases. Physical Review Letters, v. 89, n. 19, p. 190403, 2002.

47 MADISON, K. W. et al. Vortex lattices in a stirred Bose-Einstein condensate. Journal of Modern Optics, v. 47, n. 14-15, p. 2715-2723, 2000.

48 DONNELLY, R. J. Quantized vortices in helium II. Cambridge: Cambridge University Press, 1991. 368 p. ISBN 978-0-5213-2400-7.

49 LANDAU, L. D.; LIFSHITZ, E. M. Statistical physics: part 1. 3rd ed. Oxford: Oxford University Press, 1981. 561 p. (Course of theoretical physics, v. 5). ISBN 978-0-7506-3372-7. 
50 COOPER, N. R. Rapidly rotating atomic gases. Advanced in Physics, v. 57, n. 6, p. 539-616, 2008.

51 FEYNMAN, R. P. Application of quantum mechanics to liquid helium. Progress in Low Temperature Physics, v. 1, p. 17-53, 1955. doi:10.1016/S0079-6417(08)60077-3.

52 LEWENSTEIN, M. et al. Ultracold atomic gases in optical lattices: mimicking condensed matter physics and beyond. Advanced in Physics, v. 56, n. 2, p. 243-379, 2007.

53 BLOCH, I. Ultracold quantum gases in optical lattices. Nature Physics, v. 1, n. 1, p. $23-30,2005$.

54 LEWENSTEIN, M.; SAMPERA, A.; AHUFINGER, V. Ultracold atoms in optical lattices: simulating quantum many-body systems. Oxford: Oxford University Press, 2012. 496 p. ISBN 978-0-19-957312-7.

55 PETSAS, K.; A., C.; GRYNBERG, G. Crystallography of optical lattices. Physical Review A, v. 50, n. 6, p. 5173-5189, 1994.

56 SANTOS, L. et al. Atomic quantum gases in Kagome lattices. Physical Review Letters, v. 93, n. 3, p. 030601, 2004.

57 SACHDEV, S. Quantum phase transitions. 2nd ed. Cambridge: Cambridge University Press, 2001. 519 p. ISBN 978-0-521-51468-2.

58 ASHCROFT, N. W.; MERMIN N, D. Solid state physics. California: Cengage Learning Press, 1976. 848 p. ISBN 978-0-03-083993-1.

59 KITTEL, C. Introduction to solid state physics. 8th ed. New Jersey: John Wiley and Sons Press, 2005. 704 p. ISBN 978-0-471-41526-8.

60 MORSCH, O.; OBERTHALER, M. Dynamics of Bose-Einstein condensates in optical lattices. Reviews of Modern Physics, v. 78, n. 1, p. 179-215, 2006.

61 WANNIER, N. R. The structure of electronic excitation levels in insulating crystals. Physical Review, v. 52, n. 3, p. 191-197, 1937.

62 GREINER, M. et al. Exploring phase coherence in a 2D lattice of Bose-Einstein condensate. Physical Review Letters, v. 87, n. 16, p. 160405, 2001. 
63 GREINER, M. et al. Quantum phase transition from a superfluid to a Mott insulator in a gas of ultracold atoms. Nature, v. 415, n. 6867, p. 39-44, 2002.

64 GREINER, M. Ultracold atoms in optical lattice potencials. Italia. 2005. Disponível em: < http://users.physics.harvard.edu/ greiner/publications/ trieste-greiner-lattice.pdf $>$. Acesso em: 10 fev. 2016.

65 HUBBARD, J. Electron correlations in narrow energy bands. Proceedings of the Royal Society A, v. 276, n. 1365, p. 238-257, 1963.

66 MATHIEU, E. Mémoire sur le mouvement vibratoire d'une membrane de forme elliptique. Journal de Mathématiques Pures et Appliquées, v. 2, n. 13, p. 137-203, 1868.

67 BARRETT, W. Mathieu functions of general order: connection formulae, base functions and asymptotic formulae: I. introduction. Philosophical Transactions of the Royal Society of London A, v. 301, n. 1459, p. 75-79, 1981.

68 OLVER F., W. J. et al. NIST handbook of mathematical functions. Cambridge: Cambridge University Press, 2010. 967 p. ISBN 978-0-521-19225-5.

69 MEIXNER, J.; SCHÄFKE, F. W.; WOLF, G. Mathieu functions and spheroidal functions and their mathematical foundations. Berlin: Springer-Verlag Press, 1980. 140 p. ISBN 978-3-540-38411-3.

70 ABRAMOWITZ, M.; STEGUN, I. A. Handbook of mathematical functions with formulas, graphs, and mathematical tables. New York: Dover Publications, 1965. 1046 p. ISBN 978-0-486-61272-0.

71 ZWERGER, W. Mott-Hubbard transition of cold atoms in optical lattices. Journal Optics B: quantum semiclassical optics, v. 5, n. 2, p. s9-s16, 2003.

72 LEVIN, K.; FETTER, A. L.; STAMPER-KURN, D. M. Ultracold bosonic and fermionic gases. Amsterdam: Elsevier Publications, 2012. 221 p. ISBN 978-0-444-53857-4.

73 BRUUS, H.; FLENSBERG, K. Many-body quantum theory in condensed matter physics: an introduction. Oxford: Oxford University Press, 2004. 466 p. ISBN 978-0-198-56633-5. 
74 SHESHADRI, K. et al. Superfluid and insulating phases in an interacting-boson model: mean-field theory and the RPA. Europhysics Letters, v. 22, n. 4, p. 257-263, 1993.

75 OOSTEN, D. v.; STRATEN, P. v. d.; STOOF, H. T. C. Quantum phases in optical lattices. Physical Review A, v. 63, n. 5, p. 053601, 2001.

76 SCHWEIKHARD, V. et al. Rapidly rotating Bose-Einstein condensates in and near the lowest Landau level. Physical Review Letters, v. 92, n. 4, p. 040404, 2004.

77 HO, T.-L. Bose-Einstein condensates with large number of vortices. Physical Review Letters, v. 87, n. 6, p. 060403, 2001.

78 MATVENKO, S. I.; SHLYAPNIKOV, G. V. Tkachenko modes and their damping in the vortex lattice regime of rapidly rotating bosons. Physical Review A, v. 83, n. 3, p. $033604,2011$.

79 CARACANHAS, M. A.; BAGNATO, V. S.; PEREIRA, R. G. Tkachenko polarons in vortex lattices. Physical Review Letters, v. 111, n. 11, p. 115304, 2013.

80 CODDINGTON, I. et al. Experimental studies of equilibrium vortex properties in a Bose-condensed gas. Physical Review A, v. 70, n. 6, p. 063607, 2004.

81 SANTOS, F. E. A. D.; PELSTER, A. Quantum phase diagram of bosons in optical lattices. Physical Review A, v. 79, n. 1, p. 013614, 2009.

82 CAPOGROSSO-SAnsone, B.; PROKOF'EV, N. V.; SVISTUNOV, B. V. Phase diagram and thermodynamics of the three-dimensional Bose-Hubbard model. Physical Review B, v. 75, n. 13, p. 134302, 2007.

83 CAPOGROSSO-SANSONE, B. et al. Monte Carlo study of the two-dimensional Bose-Hubbard model. Physical Review A, v. 77, n. 1, p. 015602, 2008.

84 SANTOS, F. P. D. et al. Bose-Einstein condensation of metastable helium. Physical Review Letters, v. 86, n. 16, p. 3459-3462, 2001.

85 WANG, F. et al. Observation of Feshbach resonances between ultracold $\mathrm{Na}$ and $\mathrm{Rb}$ atoms. Physical Review A, v. 87, n. 5, p. 050702, 2013.

86 SAMUELIS, C. et al. Cold atomic collisions studied by molecular spectroscopy. Physical Review A, v. 63, n. 1, p. 012710, 2000. 
87 MODUGNO, G. et al. Bose-Einstein condensates of potassium atoms by symphathetic cooling. Science, v. 294, n. 5545, p. 1320-1322, 2001.

88 TAKEKOSHI, T. et al. Towards the production of ultracold ground-state Rb-Cs molecules: Feshbach resonances, weakly bound states, and the coupled-channel model. Physical Review A, v. 85, n. 3, p. 032506, 2012.

89 CHIN, C. et al. Precision Feshbach spectroscopy of ultracold $\mathrm{Cs}_{2}$. Physical Review A, v. 70, n. 3, p. 032701, 2004.

90 TKACHENKO, V. K. Elasticiy of vortex lattices. Journal of Experimental and Theoretical Physics, v. 29, n. 5, p. 945-946, 1969.

91 LANG, I. G.; FIRZOV, Y. A. Kinetic theory of semiconductors with low mobility. Journal of Experimental and Theoretical Physics, v. 16, n. 5, p. 1301-1312, 1963.

92 TREFZGER, C.; MENOTTI, C.; LEWENSTEIN, M. Ultracold dipolar gas in an optical lattice: the fate of metastable states. Physical Review A, v. 78, n. 4, p. 043604, 2008.

93 BRUDER, C.; FAZIO, R.; SCHÖN, G. Superconductor-Mott-insulator transition in Bose systems with finite-range interactions. Physical Review B, v. 47, n. 1, p. 342-347, 1993.

94 MAZZARELLA, G.; GIAMPAOLO, S. M.; ILLUMinATI, F. Extended Bose Hubbard model of interacting bosonic atoms in optical lattices: from superfluidity to density waves. Physical Review A, v. 73, n. 1, p. 013625, 2006.

95 MAHAN, G. D. Many-particle physics. New York: Plenum Press, 1981. 408 p. ISBN 0-306-40411-7. 


\section{APÊNDICE A - Hamiltoniano de}

\section{Bose-Hubbard estendido: $\alpha$ geral}

Para determinarmos o Hamiltoniano de Bose-Hubbard estendido, partimos aplicando a transformação de polaron ao Hamiltoniano total estendido. Então, aplicando a equação (6.19) em (6.20a) e depois expandindo até os primeiros vizinhos, obtemos o Hamiltoniano transformado

$$
\begin{aligned}
& \tilde{\mathrm{H}} \approx \frac{1}{\sqrt{S}} \sum_{i \mathbf{q}}\left|\alpha_{i \mathbf{q}}\right|^{2} \epsilon_{\mathbf{q}} \hat{n}_{i} \hat{n}_{i}+\frac{1}{\sqrt{S}} \sum_{\langle i j\rangle \mathbf{q}} \alpha_{j \mathbf{q}}^{*} \alpha_{i \mathbf{q}} e^{-i \mathbf{q} \cdot \mathbf{d}} \epsilon_{\mathbf{q}} \hat{n}_{i} \hat{n}_{j}-\bar{\mu}_{B} \sum_{i} \hat{n}_{i}+\frac{\mathrm{U}}{2} \sum_{i} \hat{n}_{i}\left(\hat{n}_{i}-1\right) \\
& -\frac{1}{\sqrt{S}} \sum_{j \mathbf{q}} e^{-i \mathbf{q} \cdot \mathbf{R}_{j}} \epsilon_{\mathbf{q}} \alpha_{j \mathbf{q}} \hat{n}_{j} \hat{a}_{\mathbf{q}}-\frac{1}{\sqrt{S}} \sum_{j \mathbf{q}} e^{i \mathbf{q} \cdot \mathbf{R}_{j}} \epsilon_{\mathbf{q}} \alpha_{j \mathbf{q}}^{*} \hat{n}_{j} \hat{a}_{\mathbf{q}}^{\dagger}+\sum_{\mathbf{q}} \epsilon_{\mathbf{q}} \hat{a}_{\mathbf{q}}^{\dagger} \hat{a}_{\mathbf{q}}-\mathrm{J} \sum_{\langle i j\rangle}\left(e^{-\hat{Y}_{i}^{\dagger}} e^{\hat{Y}_{j}}\right) \hat{b}_{i}^{\dagger} \hat{b}_{j} \\
& -\frac{g_{A B} \sqrt{n_{A}}}{S}\left\{\sum_{i \mathbf{q}}\left[\Omega_{\mathbf{q}}^{i i} e^{i \mathbf{q} \cdot \vec{R}_{i}} \alpha_{i \mathbf{q}}^{*}+\bar{\Omega}_{\mathbf{q}}^{i i} e^{-i \mathbf{q} \cdot \vec{R}_{i}} \alpha_{i \mathbf{q}}\right] \hat{n}_{i} \hat{n}_{i}+\sum_{\langle i j\rangle \mathbf{q}}\left[\Omega_{\mathbf{q}}^{j j} e^{i \mathbf{q} \cdot \vec{R}_{i}} \alpha_{i \mathbf{q}}^{*}+\bar{\Omega}_{\mathbf{q}}^{j j} e^{-i \mathbf{q} \cdot \vec{R}_{i}} \alpha_{i \mathbf{q}}\right] \hat{n}_{i} \hat{n}_{j}\right\} \\
& -\frac{g_{A B} \sqrt{n_{A}}}{S} \sum_{\langle i j\rangle \mathbf{q}}\left[\Omega_{\mathbf{q}}^{i j} e^{i \mathbf{q} \cdot \vec{R}_{j}} \alpha_{j \mathbf{q}}^{*}+\bar{\Omega}_{\mathbf{q}}^{i j} e^{-i \mathbf{q} \cdot \vec{R}_{j}} \alpha_{j \mathbf{q}}\right]\left(e^{-\hat{Y}_{i}^{\dagger}} e^{\hat{Y}_{j}}\right) \hat{n}_{j} \hat{b}_{i}^{\dagger} \hat{b}_{j} \\
& -\frac{g_{A B} \sqrt{n_{A}}}{S} \sum_{\langle i j\rangle \mathbf{q}}\left[\Omega_{\mathbf{q}}^{i j} e^{i \mathbf{q} \cdot \vec{R}_{i}} \alpha_{i \mathbf{q}}^{*} \bar{\Omega}_{\mathbf{q}}^{i j} e^{-i \mathbf{q} \cdot \vec{R}_{i}} \alpha_{i \mathbf{q}}\right]\left(e^{-\hat{Y}_{i}^{\dagger}} e^{\hat{Y}_{j}}\right) \hat{n}_{i} \hat{b}_{i}^{\dagger} \hat{b}_{j} \\
& +\frac{g_{A B} \sqrt{n_{A}}}{\sqrt{S}}\left\{\sum_{i \mathbf{q}} \hat{a}_{\mathbf{q}} \Omega_{\mathbf{q}}^{i i} \hat{n}_{i}+\sum_{\langle i j\rangle \mathbf{q}} \hat{a}_{\mathbf{q}}\left(e^{-\hat{Y}_{i}^{\dagger}} e^{\hat{Y}_{j}}\right) \Omega_{\mathbf{q}}^{i j} \hat{b}_{i}^{\dagger} \hat{b}_{j}+\sum_{i \mathbf{q}} \hat{a}_{\mathbf{q}}^{\dagger} \bar{\Omega}_{\mathbf{q}}^{i i} \hat{n}_{i}+\sum_{\langle i j\rangle \mathbf{q}} \hat{a}_{\mathbf{q}}^{\dagger}\left(e^{-\hat{Y}_{i}^{\dagger}} e^{\hat{Y}_{j}}\right) \bar{\Omega}_{\mathbf{q}}^{i j} \hat{b}_{i}^{\dagger} \hat{b}_{j}\right\},
\end{aligned}
$$

onde, o vetor entre dos sítios vizinhos é $\mathbf{d}=\mathbf{R}_{i}-\mathbf{R}_{j}$.

A continuação, vamos tomar a média (processo de aplicar a operação do traço sobre os graus de libertade do sistema da rede) respeito aos modos da rede com o fim de obter o Hamiltoniano do modelo de Bose-Hubbard estendido da espécie $B$.

Empregando a Eq. (6.22) sobre os operadores dos modos, teremos as seguintes relações

$$
\begin{gathered}
\left\langle f\left|\hat{a}_{\mathbf{q}}^{\dagger}\right| f\right\rangle=\left\langle f\left|\hat{a}_{\mathbf{q}}\right| f\right\rangle=0 \quad \text { e } \quad\left\langle f\left|\hat{N}_{\mathbf{q}}\right| f\right\rangle=N_{\mathbf{q}} \\
\left\langle f\left|e^{-\hat{Y}_{i}^{\dagger}} e^{\hat{Y}_{j}}\right| f\right\rangle=f^{i j} \\
\left\langle f\left|\hat{a}_{\mathbf{q}}\left(e^{-\hat{Y}_{i}^{\dagger}} e^{\hat{Y}_{j}}\right)\right| f\right\rangle=\bar{F}_{\mathbf{q}}^{i j} \\
\left\langle f\left|\hat{a}_{\mathbf{q}}^{\dagger}\left(e^{-\hat{Y}_{i}^{\dagger}} e^{\hat{Y}_{j}}\right)\right| f\right\rangle=F_{\mathbf{q}}^{i j}
\end{gathered}
$$


Ao substituir as equações (A.2a)-(A.2d) em (A.1), deduz-se o Hamiltoniano de BoseHubbard estendido da espécie $B$

$$
\mathbf{H} \approx-\tilde{\mathrm{J}} \sum_{\langle i j\rangle} \hat{b}_{i}^{\dagger} \hat{b}_{j}+\frac{\tilde{\mathrm{U}}}{2} \sum_{i} \hat{n}_{i}\left(\hat{n}_{i}-1\right)-\tilde{\mu} \sum_{i} \hat{n}_{i}+\sum_{\langle i j\rangle} \frac{\mathbf{V}_{i j}}{2} \hat{n}_{i} \hat{n}_{j}-\sum_{\langle i j\rangle} \mathbf{P}_{i j} \hat{n}_{j} \hat{b}_{i}^{\dagger} \hat{b}_{j}-\sum_{\langle i j\rangle} \mathbf{Q}_{i j} \hat{n}_{i} \hat{b}_{i}^{\dagger} \hat{b}_{j}
$$

com

$$
\begin{aligned}
\tilde{\mathrm{J}} & =\left\{J-\frac{g_{A B} \sqrt{n_{A}}}{\sqrt{S}} \sum_{\mathbf{q}}\left(e^{i \mathbf{q} \cdot \mathbf{R}_{i}}\left[\alpha_{\mathbf{q} i}^{*}-e^{-i \mathbf{q} \cdot \mathbf{d}} \alpha_{\mathbf{q} j}^{*}\right] \Omega_{\mathbf{q}}^{i j}+e^{-i \mathbf{q} \cdot \mathbf{R}_{i}}\left[\alpha_{\mathbf{q} i}-e^{i \mathbf{q} \cdot \mathbf{d}} \alpha_{\mathbf{q} j}\right] \bar{\Omega}_{\mathbf{q}}^{i j}\right)\right\} f^{0} \\
\tilde{\mathrm{U}} & =U+\frac{2}{S} \sum_{\mathbf{q}}\left[\left|\alpha_{i \mathbf{q}}\right|^{2} \epsilon_{\mathbf{q}}-g_{A B} \sqrt{n_{A}}\left(\Omega_{i \mathbf{q}} e^{i \mathbf{q} \cdot \mathbf{R}_{i}} \alpha_{i \mathbf{q}}^{*}+\Omega_{i \mathbf{q}}^{*} e^{-i \mathbf{q} \cdot \mathbf{R}_{i}} \alpha_{i \mathbf{q}}\right)\right] \\
\tilde{\mu} & =\mu-\frac{1}{S} \sum_{\mathbf{q}}\left[\left|\alpha_{i \mathbf{q}}\right|^{2} \epsilon_{\mathbf{q}}-g_{A B} \sqrt{n_{A}}\left(\Omega_{i \mathbf{q}} e^{i \mathbf{q} \cdot \mathbf{R}_{i}} \alpha_{i \mathbf{q}}^{*}+\Omega_{i \mathbf{q}}^{*} e^{-i \mathbf{q} \cdot \mathbf{R}_{i}} \alpha_{i \mathbf{q}}\right)\right] \\
\mathrm{V}_{i j} & =\frac{2}{S} \sum_{\mathbf{q}}\left[\alpha_{j \mathbf{q}}^{*} \alpha_{i \mathbf{q}} e^{-i \mathbf{q} \cdot \mathbf{d}} \epsilon_{\mathbf{q}}-g_{A B} \sqrt{n_{A}}\left(\Omega_{j \mathbf{q}} e^{i \mathbf{q} \cdot \mathbf{R}_{i}} \alpha_{i \mathbf{q}}^{*}+\Omega_{j \mathbf{q}}^{*} e^{-i \mathbf{q} \cdot \mathbf{R}_{i}} \alpha_{i \mathbf{q}}\right)\right] \\
\mathrm{P}_{i j} & =\frac{g_{A B} \sqrt{n_{A}}}{S} \sum_{\mathbf{q}}\left[\Omega_{\mathbf{q}}^{i j} e^{i \mathbf{q} \cdot \mathbf{R}_{j}} \alpha_{j \mathbf{q}}^{*}+\bar{\Omega}_{\mathbf{q}}^{i j} e^{-i \mathbf{q} \cdot \mathbf{R}_{j}} \alpha_{j \mathbf{q}}\right] f^{0} \\
\mathrm{Q}_{i j} & =\frac{g_{A B} \sqrt{n_{A}}}{S} \sum_{\mathbf{q}}\left[\Omega_{\mathbf{q}}^{i j} e^{i \mathbf{q} \cdot \mathbf{R}_{i}} \alpha_{i \mathbf{q}}^{*}+\bar{\Omega}_{\mathbf{q}}^{i j} e^{-i \mathbf{q} \cdot \mathbf{R}_{i}} \alpha_{i \mathbf{q}}\right] f^{0}
\end{aligned}
$$

onde $\Omega_{\mathbf{q}}^{i j}=\Omega_{i \mathbf{q}}$ e $\bar{\Omega}_{\mathbf{q}}^{i j}=\Omega_{i \mathbf{q}}^{*}$ é obtido fazendo $i=j$ na Eq. $(6.17 \mathrm{c})$. 


\section{APÊNDICE B - Parâmetros associados aos}

\section{modos}

A continuação vamos computar os parâmetros que surgem após aplicar o traço ao Hamiltoniano de Bose-Hubbar estendido, Eq. (6.22), uma vez aplicada a transformação unitária.

Definindo os seguintes operadores

$$
\hat{x}_{i}=e^{\hat{Y}_{i}} \quad \text { e } \quad \hat{x}_{i}^{\dagger}=e^{-\hat{Y}_{i}}
$$

Empregando a formula de Baxer-Campbell-Hausdorff: $e^{\hat{A}} e^{\hat{B}}=e^{\hat{A}+\hat{B}+\frac{1}{2}[\hat{A}, \hat{B}]}$, na Eq. (B.1), tem-se

$$
\hat{x}_{i}^{\dagger} \hat{x}_{j}=e^{-\left(\hat{Y}_{i}+\hat{Y}_{i}\right)-\frac{1}{2}\left[\hat{Y}_{i}, \hat{Y}_{i}\right]}=e^{-\hat{\mathrm{U}}} .
$$

Utilizando a equação (6.21b) em (B.2a)

$$
\begin{aligned}
\hat{\mathrm{U}} & =\frac{1}{2}\left[\frac{1}{\sqrt{S}} \sum_{\mathbf{q}}\left[e^{-i \mathbf{q} \cdot \mathbf{R}_{i}} \alpha_{\mathbf{q} i} \hat{a}_{\mathbf{q}}-e^{i \mathbf{q} \cdot \mathbf{R}_{i}} \alpha_{\mathbf{q} i}^{*} \hat{a}_{\mathbf{q}}^{\dagger}\right], \frac{1}{\sqrt{S}} \sum_{\mathbf{q}^{\prime}}\left[e^{-i \mathbf{q}^{\prime} \cdot \mathbf{R}_{j}} \alpha_{\mathbf{q}^{\prime} j} \hat{a}_{\mathbf{q}^{\prime}}-e^{i \mathbf{q}^{\prime} \cdot \mathbf{R}_{j}} \alpha_{\mathbf{q}^{\prime} j}^{*} \hat{a}_{\mathbf{q}}^{\dagger}\right]\right] \\
& +\frac{1}{\sqrt{S}} \sum_{\mathbf{q}}\left[e^{-i \mathbf{q} \cdot \mathbf{R}_{i}} \alpha_{\mathbf{q} i} \hat{a}_{\mathbf{q}}-e^{i \mathbf{q} \cdot \mathbf{R}_{i}} \alpha_{\mathbf{q} i}^{*} \hat{a}_{\mathbf{q}}^{\dagger}\right]-\frac{1}{\sqrt{S}} \sum_{\mathbf{q}}\left[e^{-i \mathbf{q} \cdot \mathbf{R}_{j}} \alpha_{\mathbf{q} j} \hat{a}_{\mathbf{q}}-e^{i \mathbf{q} \cdot \mathbf{R}_{j}} \alpha_{\mathbf{q} j}^{*} \hat{a}_{\mathbf{q}}^{\dagger}\right] \\
\hat{\mathrm{U}} & =\frac{1}{\sqrt{S}} \sum_{\mathbf{q}}\left[(\hat{a}_{\mathbf{q}}-\underbrace{\frac{\alpha_{\mathbf{q} i}^{*}}{2 \sqrt{S}} e^{i \mathbf{q} \cdot \mathbf{R}_{i}}}_{\bar{a}_{\mathbf{q}}}) \lambda_{\mathbf{q}}^{i j}-(\hat{a}_{\mathbf{q}}^{\dagger}-\underbrace{\frac{\alpha_{\mathbf{q} i}}{2 \sqrt{S}} e^{-i \mathbf{q} \cdot \mathbf{R}_{i}}}_{a_{\mathbf{q}}}) \lambda_{\mathbf{q}}^{* i j}\right] .
\end{aligned}
$$

com

$$
\lambda_{\mathbf{q}}^{i j}=e^{-i \mathbf{q} \cdot \mathbf{R}_{i}}\left[\alpha_{\mathbf{q} i}-e^{i \mathbf{q} \cdot \mathbf{d}} \alpha_{\mathbf{q} j}\right] \quad \text { e } \quad \lambda_{\mathbf{q}}^{* i j}=e^{i \mathbf{q} \cdot \mathbf{R}_{i}}\left[\alpha_{\mathbf{q} i}^{*}-e^{-i \mathbf{q} \cdot \mathbf{d}} \alpha_{\mathbf{q} j}^{*}\right]
$$

sendo $\bar{a}_{\mathbf{q}}, a_{\mathbf{q}}$ escalares que representam simples deslocamentos dos operadores de criação e destruição. Pode-se obviar esses termos sem perder generalidade. Fazendo isso, tem-se

$$
\hat{\mathrm{U}} \equiv \frac{1}{\sqrt{S}} \sum_{\mathbf{q}}\left[\hat{a}_{\mathbf{q}} \lambda_{\mathbf{q}}^{i j}-\hat{a}_{\mathbf{q}}^{\dagger} \lambda_{\mathbf{q}}^{* i j}\right]
$$

Então, substituindo a equação (B.2d) em (B.2a); e logo empregando o teorema $e^{\hat{A}+\hat{B}} \approx$ $e^{\hat{A}} e^{\hat{B}} e^{\frac{1}{2}[\hat{A}, \hat{B}]}$, tem-se

$$
\hat{x}_{i}^{\dagger} \hat{x}_{j} \approx \prod_{\mathbf{q}} e^{-\frac{1}{2}\left|\mho_{\mathbf{q}}^{i j}\right|^{2}} e^{\mho_{\mathbf{q}}^{* i j} \hat{a}_{\mathbf{q}}^{\dagger}} e^{-\mho_{\mathbf{q}}^{i j} \hat{a}_{\mathbf{q}}}
$$


Com

$$
\mho_{\mathbf{q}}^{i j}=\frac{\lambda_{\mathbf{q}}^{i j}}{\sqrt{S}} \quad \text { e } \quad \mho_{\mathbf{q}}^{* i j}=\frac{\lambda_{\mathbf{q}}^{* i j}}{\sqrt{S}}
$$

\section{- Parâmetro $f^{i j}$}

Aplicando a equação (B.2e) em (A.2b)

$$
f^{i j}=\left\langle f\left|\hat{x}_{i}^{\dagger} \hat{x}_{j}\right| f\right\rangle \approx \prod_{\mathbf{q}} e^{-\frac{1}{2}\left|\mho_{\mathbf{q}}^{i j}\right|^{2}} \underbrace{\left\langle f\left|e^{\mho_{\mathbf{q}}^{* i j} \hat{a}_{\mathbf{q}}^{\dagger}} e^{-\mho_{\mathbf{q}}^{i j} \hat{a}_{\mathbf{q}}}\right| f\right\rangle} .
$$

O formalismo efetuado por Mahan (95) (Ele emprega as funções de Matsubara para o "tempo complexo", onde $i t \rightarrow \tau$ ) para a expressão entre chaves é

$$
\begin{aligned}
\left(1-e^{\beta \epsilon_{\mathbf{q}}}\right) \sum_{N_{\mathbf{q}}}^{\infty} e^{\beta N_{\mathbf{q}} \epsilon_{\mathbf{q}}}\left\langle N_{\mathbf{q}}\right| e^{u_{\mathbf{q}}^{*} \hat{a}_{\mathbf{q}}^{\dagger} e^{-u \hat{a}_{\mathbf{q}}\left|N_{\mathbf{q}}\right\rangle}}=e^{-|u|^{2} \hat{N}_{\mathbf{q}}} \\
\hat{N}_{\mathbf{q}}=1 /\left(e^{\beta \epsilon_{\mathbf{q}}}-1\right) .
\end{aligned}
$$

Aplicando a equação (B.4a) em (B.3), tem-se

$$
f^{i j} \approx\left\langle f\left|\hat{x}_{i}^{\dagger} \hat{x}_{j}\right| f\right\rangle \approx e^{-\frac{1}{S} \sum_{\mathbf{q}}\left|\delta_{\mathbf{q}}^{i j}\right|^{2}\left(N_{\mathbf{q}}-\frac{1}{2}\right)}
$$

Para $T \rightarrow 0$, usando as Eqs. (A.2b) e (B.4c), obtém-se

$$
f^{i j} \approx\left\langle f\left|e^{-\hat{Y}_{i}^{\dagger}} e^{\hat{Y}_{j}}\right| f\right\rangle \approx e^{-\frac{1}{2 S} \sum_{\mathbf{q}}\left|\delta_{\mathbf{q}}^{i j}\right|^{2}}
$$

O modulo do parâmetro $\mho$ vira independente da fase associada ao sítio: $\left|\mho_{\mathbf{q}}^{i j}\right|^{2}=\left(\alpha_{\mathbf{q} i}^{*}-\right.$ $\left.e^{-i \mathbf{q} \cdot \mathbf{d}} \alpha_{\mathbf{q} j}^{*}\right)\left(\alpha_{\mathbf{q} i}-e^{i \mathbf{q} \cdot \mathbf{d}} \alpha_{\mathbf{q} j}\right)$. Nota-se que ao trocar os índices o módulo mantém sua forma, a diferença do caso linear. Como consequência deste fato, o módulo fica independente dos índices, então

$$
f^{0}=f^{i j} \approx e^{-\frac{1}{2 S} \sum_{\mathbf{q}}\left|\alpha_{0 \mathbf{q}}\left(1-e^{i \mathbf{q} \cdot \mathbf{d}}\right)\right|^{2}} .
$$

\section{- Parâmetro $\bar{F}_{\mathbf{q}}^{i j}$ :}

Expandindo a equação (B.2e)

$$
\hat{x}_{i}^{\dagger} \hat{x}_{j} \approx\left[\prod_{\mathbf{q} \neq \mathbf{q}^{\prime}} e^{-\frac{1}{2}\left|\mho_{\mathbf{q}}^{i j}\right|^{2}} e^{\mho \mho_{\mathbf{q}}^{* i j} \hat{a}_{\mathbf{q}}^{\dagger}} e^{-\mho_{\mathbf{q}}^{i j} \hat{a}_{\mathbf{q}}}\right] e^{-\frac{1}{2}\left|\mho_{\mathbf{q}^{\prime}}^{i j}\right|^{2}} e^{\mho_{\mathbf{q}^{\prime}}^{* i j} \hat{a}_{\mathbf{q}^{\prime}}^{\dagger}} e^{-\mho_{\mathbf{q}^{\prime}}^{i j} \hat{a}_{\mathbf{q}^{\prime}}}
$$

Aplicando $|f\rangle=\left|\hat{n}_{\mathbf{q}} ; \hat{n}_{\mathbf{q}^{\prime}}\right\rangle$ à equação (B.6a) e empregando o formalismo de Mahan para cada fator de

$$
\left\langle\hat{a}_{\mathbf{q}^{\prime}}\left(\hat{x}_{i}^{\dagger} \hat{x}_{j}\right)\right\rangle=\left[\prod_{\mathbf{q} \neq \mathbf{q}^{\prime}} e^{-\left|\gamma_{\mathbf{q}^{\prime}}^{i j}\right|^{2}\left(N_{\mathbf{q}}+1 / 2\right)}\right] e^{-\frac{1}{2}\left|\gamma_{\mathbf{q}^{\prime}}^{i j}\right|^{2}}\left(1-e^{\beta \epsilon_{\mathbf{q}^{\prime}}}\right) \sum_{N_{\mathbf{q}^{\prime}}}^{\infty} e^{\beta N_{\mathbf{q}^{\prime}} \epsilon_{\mathbf{q}^{\prime}}}\left\langle N_{\mathbf{q}^{\prime}}\left|\hat{a}_{\mathbf{q}^{\prime}} e^{\mho \mho_{\mathbf{q}^{\prime}}^{* i j} \hat{a}_{\mathbf{q}^{\prime}}^{\dagger}} e^{-\mho_{\mathbf{q}^{\prime}}^{i j} \hat{a}_{\mathbf{q}^{\prime}}}\right| N_{\mathbf{q}^{\prime}}\right\rangle,
$$


onde será feito $\left\langle N_{\mathbf{q}^{\prime}}\right| \hat{a}_{\mathbf{q}^{\prime}}^{\dagger}=\sqrt{N_{\mathbf{q}^{\prime}}+1}\left\langle N_{\mathbf{q}^{\prime}}+1\right|$.

Expandindo as exponenciais

$$
e^{\mho_{\mathbf{q}}^{* i j} \hat{a}_{\mathbf{q}}^{\dagger}}=\sum_{l=0}^{N_{\mathbf{q}}} \frac{\left(\mho_{\mathbf{q}}^{* i j}\right)^{l}}{l !}\left(\hat{a}_{\mathbf{q}}^{\dagger}\right)^{l} \quad \text { e } \quad e^{-\mho_{\mathbf{q}}^{i j} \hat{a}_{\mathbf{q}}}=\sum_{m=0}^{N_{\mathbf{q}}} \frac{\left(-\mho_{\mathbf{q}}^{i j}\right)^{m}}{m !}\left(\hat{a}_{\mathbf{q}}\right)^{m}
$$

Onde assume-se o mesmo critério que Mahan para $\left(\hat{a}_{\mathbf{q}}^{\dagger}\right)^{l}=\left(\hat{a}_{\mathbf{q}}^{\dagger}\right)^{m}=0$ para todo $l, m>N_{\mathbf{q}}$. Ao aplicar o estado $\left|N_{\mathbf{q}^{\prime}}\right\rangle$ simultaneamente na equação (B.6c), consegue-se

$$
e^{\mho_{\mathbf{q}^{\prime}}^{* i j} \hat{a}_{\mathbf{q}^{\prime}}^{\dagger}} e^{-\mho_{\mathbf{q}^{\prime}}^{i j} \hat{a}_{\mathbf{q}^{\prime}}}\left|N_{\mathbf{q}^{\prime}}\right\rangle=\sum_{l, m=0}^{N_{\mathbf{q}^{\prime}}} \frac{\left(\mho_{\mathbf{q}^{\prime}}^{* i j}\right)^{l}}{l !} \frac{\left(-\mho_{\mathbf{q}^{\prime}}^{i j}\right)^{m}}{m !} \sqrt{\prod_{i=0}^{m-1}\left(N_{\mathbf{q}^{\prime}}-i\right) \prod_{j=m-1}^{m-l}\left(N_{\mathbf{q}^{\prime}}-j\right)}\left|N_{\mathbf{q}^{\prime}}-m+l\right\rangle .
$$

Aplicando o estado $\left\langle N_{\mathbf{q}^{\prime}}+1\right|$ na equação (B.6d)

$$
\left\langle N_{\mathbf{q}^{\prime}}+1\left|e^{\mho \mho_{\mathbf{q}^{\prime}}^{* i j} \hat{a}_{\mathbf{q}^{\prime}}^{\dagger}} e^{-\mho_{\mathbf{q}^{\prime}}^{i j} \hat{a}_{\mathbf{q}^{\prime}}}\right| N_{\mathbf{q}^{\prime}}-m+l\right\rangle=\sum_{m=0}^{N_{\mathbf{q}^{\prime}}} \mho_{\mathbf{q}^{\prime}}^{* i j} \frac{(-1)^{m}}{(m+1) !} \frac{\left|\mho_{\mathbf{q}^{\prime}}^{i j}\right|^{2 m}}{m !} \frac{\left(N_{\mathbf{q}^{\prime}}+1\right) N_{\mathbf{q}^{\prime}} !}{\left(N_{\mathbf{q}^{\prime}}-m\right) !}
$$

onde foi efetuado $\left\langle N_{\mathbf{q}^{\prime}}+1 \mid N_{\mathbf{q}^{\prime}-m+l}\right\rangle=\delta_{N_{\mathbf{q}^{\prime}}+1, N_{\mathbf{q}^{\prime}}-m+l}=\delta_{1,-m+l}$, então $l=m+1$.

Substituindo a equação (B.6e) em (B.6b), tem-se

$$
\begin{aligned}
\left\langle f\left|\hat{a}_{\mathbf{q}^{\prime}\left(\hat{x}_{i}^{\dagger} \hat{x}_{j}\right)}\right| f\right\rangle & =\left[\prod_{\mathbf{q}_{\mathbf{q} \mathbf{q}^{\prime}}} e^{-\left|\mho_{\mathbf{q}}^{i j}\right|^{2}\left(N_{\mathbf{q}^{+1}}+2\right)}\right] e^{-\frac{1}{2}\left|\mho_{\mathbf{q}^{\prime}}^{i j}\right|^{2}}\left(\mho_{\mathbf{q}^{\prime}}^{* i j}\right)\left(1-e^{\beta \epsilon_{\mathbf{q}^{\prime}}}\right) \times \\
& \sum_{N_{\mathbf{q}^{\prime}}}^{\infty} e^{\beta N_{\mathbf{q}^{\prime}} \epsilon_{\mathbf{q}^{\prime}}} \underbrace{\sum_{m=0}^{N_{\mathbf{q}^{\prime}}} \frac{(-1)^{m}}{(m+1) !} \frac{\left|\mho_{\mathbf{q}^{\prime}}^{i j}\right|^{2 m}}{m !} \frac{\left(N_{\mathbf{q}^{\prime}}+1\right) N_{\mathbf{q}^{\prime}} !}{\left(N_{\mathbf{q}^{\prime}}-m\right) !}}_{(*)} .
\end{aligned}
$$

Seja o polinômio associado de Laguerre e sua função geradora

$$
L_{n}^{k}(x)=\sum_{n=0}^{\infty} \frac{(-1)^{m}}{m !} \frac{(n+k) !}{(m+k) !} \frac{x^{m}}{(n-m) !}, k>-1 \quad \text { e } \quad \frac{e^{-x z / 1-z}}{(1-z)^{k+1}}=\sum_{n=0}^{\infty} L_{n}^{k}(x) z^{n},|z|<1 .
$$

Utilizando a equação (B.6g) em (*) de (B.6e), obtém-se

$$
\bar{F}_{\mathbf{q}^{\prime}}^{i j}=\left\langle f\left|\hat{a}_{\mathbf{q}^{\prime}}\left(\hat{x}_{i}^{\dagger} \hat{x}_{j}\right)\right| f\right\rangle=\mho_{\mathbf{q}^{\prime}}^{* i j}\left(N_{\mathbf{q}^{\prime}}+1\right) e^{-\left|\mho_{\mathbf{q}^{\prime}}^{i j}\right|^{2}\left(N_{\mathbf{q}^{\prime}}+1 / 2\right)},
$$

Onde foi trocado $\mathbf{q}^{\prime} \rightarrow \mathbf{q}$ sem perder generalidade.

Para $T \rightarrow 0$, usando as Eqs. (A.2c) e (B.6h) em (B.6f), deriva-se

$$
\bar{F}_{\mathbf{q}}^{i j}=\left\langle f\left|\hat{a}_{\mathbf{q}}\left(e^{-\hat{Y}_{i}^{\dagger}} e^{\hat{Y}_{j}}\right)\right| f\right\rangle=\frac{\mho_{\mathbf{q}}^{* i j}}{\sqrt{S}} f^{0}
$$

- Parâmetro $F_{\mathbf{q}}^{i j}$ : 
Aplicando $|f\rangle=\left|\hat{n}_{\mathbf{q}} ; \hat{n}_{\mathbf{q}^{\prime}}\right\rangle$ à equação (A.2a) e empregando o formalismo de Mahan a cada fator de

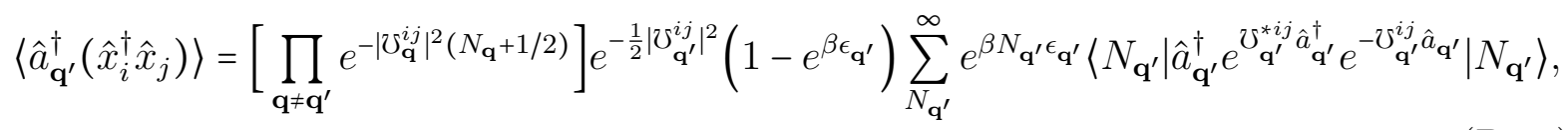

onde será feito $\left\langle N_{\mathbf{q}^{\prime}}\right| \hat{a}_{\mathbf{q}^{\prime}}=\sqrt{N_{\mathbf{q}^{\prime}}}\left\langle N_{\mathbf{q}^{\prime}}-1\right|$.

Aplicando o estado $\left\langle N_{\mathbf{q}^{\prime}}-1\right|$, e usando a equação (B.6d) em (B.8a), atinge-se

$$
\begin{aligned}
\left\langle f\left|\hat{a}_{\mathbf{q}^{\prime}\left(\hat{x}_{i}^{\dagger} \hat{x}_{j}\right)}^{\dagger}\right| f\right\rangle & =\left[\prod_{\mathbf{q}^{\neq} \mathbf{q}^{\prime}} e^{-\left|\delta_{\mathbf{q}^{\prime}}^{i j}\right|^{2}\left(N_{\left.\mathbf{q}^{+}+1 / 2\right)}\right.}\right] e^{-\frac{1}{2}\left|\gamma_{\mathbf{q}^{\prime}}^{i j}\right|^{2}}\left(-\mho_{\mathbf{q}^{\prime}}^{i j}\right)\left(1-e^{\beta \epsilon_{\mathbf{q}^{\prime}}}\right) \times \\
& \sum_{N_{\mathbf{q}^{\prime}}}^{\infty} e^{\beta N_{\mathbf{q}^{\prime}} \epsilon_{\mathbf{q}^{\prime}}} \underbrace{N_{\mathbf{q}^{\prime}} \frac{(-1)}{l} \frac{(-1)}{(l+1) !} \frac{\left|\mho_{\mathbf{q}^{\prime}}^{i j}\right|^{2 l}}{l !} \frac{\left(N_{\mathbf{q}^{\prime}}+1\right) N_{\mathbf{q}^{\prime}} !}{\left(N_{\mathbf{q}^{\prime}}-l\right) !}},
\end{aligned}
$$

onde usou-se $\left\langle N_{\mathbf{q}^{\prime}}-1 \mid N_{\mathbf{q}^{\prime}-m+l}\right\rangle=\delta_{N_{\mathbf{q}^{\prime}}-1, N_{\mathbf{q}^{\prime}}-m+l}=\delta_{-1,-m+l}$, então $m=l+1$.

Utilizando as equações (B.6g) em (*) de (B.8b), tem-se

$$
F_{\mathbf{q}}^{i j}=\left\langle f\left|\hat{a}_{\mathbf{q}}^{\dagger}\left(\hat{x}_{i}^{\dagger} \hat{x}_{j}\right)\right| f\right\rangle=-\mho_{\mathbf{q}}^{i j}\left(N_{\mathbf{q}}+1\right) e^{-\left|\mho_{\mathbf{q}}^{i j}\right|^{2}\left(N_{\mathbf{q}}+1 / 2\right)} .
$$

Onde foi trocado $\mathbf{q}^{\prime} \rightarrow \mathbf{q}$ sem perder generalidade.

Para $T \rightarrow 0$, empregando as Eqs. (A.2d) e (B.8c), deduz-se

$$
F_{\mathbf{q}}^{i j}=\left\langle f\left|\hat{a}_{\mathbf{q}}^{\dagger}\left(e^{-\hat{Y}_{i}^{\dagger}} e^{\hat{Y}_{j}}\right)\right| f\right\rangle=-\frac{\mho_{\mathbf{q}}^{i j}}{\sqrt{S}} f^{0} .
$$




\section{APÊNDICE C - Aproximação de MF da}

\section{TFQ}

Considerando $\mathbf{V}=-\tilde{\mathbf{V}}(>0)$, o Hamiltoniano de Bose-Hubbard estendido da espécie $B$, Eq. (6.27), fica

$$
\mathbf{H} \approx-\tilde{\mathbf{J}} \sum_{\langle i j\rangle} \hat{b}_{i}^{\dagger} \hat{b}_{j}+\frac{1}{2} \tilde{\mathbf{U}} \sum_{i} \hat{n}_{i}\left(\hat{n}_{i}-1\right)-\tilde{\mu}_{B} \sum_{i} \hat{n}_{i}-\frac{1}{2} \tilde{\mathbf{V}} \sum_{\langle i j\rangle} \hat{n}_{i} \hat{n}_{j}
$$

Seja a transformação de MF tanto para o operador de criação (destruição) como o operador número

$$
\begin{aligned}
& \left\{\begin{array}{l}
\hat{b}_{i}=\phi_{i}+\delta \hat{b}_{i} \\
\hat{b}_{i}^{\dagger}=\phi_{i}^{*}+\delta \hat{b}_{i}^{\dagger}
\end{array}\right. \\
& \hat{n}_{i}=\left\langle\hat{n}_{i}\right\rangle+\delta \hat{n}_{i},
\end{aligned}
$$

onde $\phi_{i}=\left\langle\hat{b}_{i}\right\rangle$ é definido como parâmetro de ordem e $n_{i}=\left\langle\hat{n}_{i}\right\rangle$ é a densidade dos sítios na rede. Empregando as relações de acima, obtemos

$$
\begin{aligned}
\hat{b}_{i}^{\dagger} \hat{b}_{j} & \approx \hat{b}_{i}^{\dagger} \phi_{j}+\hat{b}_{j} \phi_{i}^{*}-\phi_{i}^{*} \phi_{j} \\
\left\langle\hat{n}_{i}\right\rangle & \approx\left\langle\hat{b}_{i}^{\dagger}\right\rangle\left\langle\hat{b}_{i}\right\rangle \\
\hat{n}_{i} \hat{n}_{j} & \approx \hat{n}_{i}\left\langle\hat{n}_{j}\right\rangle+\hat{n}_{j}\left\langle\hat{n}_{i}\right\rangle-\left\langle\hat{n}_{i}\right\rangle\left\langle\hat{n}_{j}\right\rangle,
\end{aligned}
$$

onde omitimos os termos de ordem quadrático da flutuação.

Os termos associados à energia de tunelamento, Eq. (C.3a), e potencial de longo alcançe, Eq. (C.3c), podem ser reescritos da seguinte forma

$$
\begin{aligned}
& \sum_{\langle i j\rangle} \hat{b}_{i}^{\dagger} \hat{b}_{j} \approx \sum_{\langle i j\rangle}\left[\hat{b}_{i}^{\dagger} \phi_{j}+\hat{b}_{j} \phi_{i}^{*}-\phi_{i}^{*} \phi_{j}\right] \approx \sum_{i}\left[\hat{b}_{i}^{\dagger} \tilde{\phi}_{i}+\hat{b}_{i} \tilde{\phi}_{i}^{*}-\phi_{i}^{*} \tilde{\phi}_{i}\right] \\
& \sum_{\langle i j\rangle} \hat{n}_{i} \hat{n}_{j} \approx \sum_{\langle i j\rangle}\left[2 \hat{n}_{i}\left\langle\hat{n}_{j}\right\rangle-\left\langle\hat{n}_{i}\right\rangle\left\langle\hat{n}_{j}\right\rangle\right] \approx \sum_{i}\left[2 \tilde{n}_{i} \hat{n}_{i}-n_{i} \tilde{n}_{i}\right]
\end{aligned}
$$

com

$$
\tilde{\phi}_{i}=\sum_{\langle i\rangle_{j}}\left\langle\hat{b}_{i}\right\rangle, \quad \tilde{\phi}_{i}^{*}=\sum_{\langle i\rangle_{j}}\left\langle\hat{b}_{i}^{\dagger}\right\rangle \quad \text { e } \quad \tilde{n}_{i}=\sum_{\langle i\rangle_{j}} n_{j}
$$


onde $\langle i\rangle_{j}$ representa o somatório sobre o número total de primeiros vizinhos.

Utilizando as equações anteriores, deriva-se o Hamiltoniano de MF de um sítio

$$
\mathbf{H}^{M F}=\sum_{i} \mathbf{H}_{i}^{M F}=\sum_{i} \mathbf{H}_{i}^{p e r}+\sum_{i} \mathbf{H}_{i}^{0},
$$

com

$$
\begin{aligned}
\mathbf{H}_{i}^{\text {per }} & =-\tilde{\mathbf{J}}\left[\hat{b}_{i}^{\dagger} \tilde{\phi}_{i}+\hat{b}_{i} \tilde{\phi}_{i}^{*}\right] \\
\mathbf{H}_{i}^{0} & =\frac{1}{2} \tilde{\mathbf{U}} \hat{n}_{i}\left(\hat{n}_{i}-1\right)-\tilde{\mu} \hat{n}_{i}-\frac{1}{2} \tilde{\mathbf{V}}\left[2 \tilde{n}_{i} \hat{n}_{i}-n_{i} \tilde{n}_{i}\right]+\tilde{\mathbf{J}} \phi_{i}^{*} \tilde{\phi}_{i},
\end{aligned}
$$

onde, a primeira relação pode ser entendida como uma "perturbação" do sistema (tunelamento pequeno em comparação com as outras energias), enquanto que o estado fundamental é estabelecida pela segunda expressão.

A energia $E_{i}$ do estado fundamental associada ao $i$-ésimo sítio, pode ser obtida a partir do Hamiltoniano não perturbado

$$
\mathbf{H}_{i}^{0}\left|n_{i}\right\rangle=E_{n_{i}}^{0}\left|n_{i}\right\rangle
$$

Para determinar a TFQ, vamos empregar a teoria de perturbações, a qual será expandida até segundo ordem em $\mathbf{H}_{i}^{\text {per }}$, então

$$
E_{n} \approx E_{n_{i}}^{0}+\left\langle n_{i}\left|\mathbf{H}_{i}^{\text {per }}\right| n_{i}\right\rangle+\sum_{m_{i} \neq n_{i}} \frac{\left|\left\langle m_{i}\left|\mathbf{H}_{i}^{p e r}\right| n_{i}\right\rangle\right|^{2}}{\left(E_{n_{i}}^{0}-E_{m_{i}}^{0}\right)}
$$

Computando cada termo

$$
\begin{aligned}
\left\langle n_{i}\left|\mathbf{H}_{i}^{\text {per }}\right| n_{i}\right\rangle= & -\tilde{\mathbf{J}}\left\langle n_{i}\left|\hat{b}_{i}^{\dagger} \tilde{\phi}_{i}+\hat{b}_{i} \tilde{\phi}_{i}^{*}\right| n_{i}\right\rangle=0, \\
\left|\left\langle m_{i}\left|\mathbf{H}_{i}^{\text {per }}\right| n_{i}\right\rangle\right|^{2}= & \left|-\tilde{\mathbf{J}} \tilde{\phi}_{i}\left\langle m_{i}\left|\hat{b}_{i}^{\dagger}\right| n_{i}\right\rangle-\tilde{\mathbf{J}} \tilde{\phi}_{i}^{*}\left\langle m_{i}\left|\hat{b}_{i}\right| n_{i}\right\rangle\right|^{2} \\
= & \tilde{\mathbf{J}}^{2}\left|\tilde{\phi}_{i}\right|^{2}\left[n_{i} \delta_{m_{i}, n_{i}-1}+\left(n_{i}+1\right) \delta_{m_{i}, n_{i}+1}\right] \\
& +\tilde{\mathbf{J}}^{2} \sqrt{n_{i}\left(n_{i}+1\right)}\left[\tilde{\phi}_{i}^{* 2} \delta_{m_{i}, n_{i}+1}+\tilde{\phi}_{i}^{2} \delta_{m_{i}, n_{i}-1}\right] .
\end{aligned}
$$

Empregando a equação (C.8b), obtemos

$$
\sum_{m_{i} \neq n_{i}} \frac{\left|\left\langle m_{i}\left|\mathbf{H}_{i}^{p e r}\right| n_{i}\right\rangle\right|^{2}}{\left(E_{n_{i}}^{0}-E_{m_{i}}^{0}\right)}=\tilde{\mathbf{J}}^{2}\left|\tilde{\phi}_{i}\right|^{2}\left\{\frac{n_{i}+1}{E_{n_{i}}^{0}-E_{n_{i}+1}^{0}}+\frac{n_{i}}{E_{n_{i}}^{0}-E_{n_{i}-1}^{0}}\right\} .
$$


As energias são obtidas empregando a equação de auto-estados (C.6d), da qual, obtemos

$$
\begin{gathered}
\mathbf{H}_{i}^{0}\left|n_{i}\right\rangle=E_{n_{i}}^{0}\left|n_{i}\right\rangle \Rightarrow E_{n_{i}}^{0}=\frac{\tilde{\mathbf{U}}}{2} n_{i}\left(n_{i}-1\right)-\tilde{\mu} n_{i}-\frac{\tilde{\mathbf{V}}}{2} \tilde{n}_{i} n_{i}+\tilde{\mathbf{J}} \phi_{i}^{*} \tilde{\phi}_{i}, \\
\mathbf{H}_{i}^{0}\left|n_{i}+1\right\rangle=E_{n_{i}+1}^{0}\left|n_{i}+1\right\rangle \Rightarrow E_{n_{i}+1}^{0}=\frac{\tilde{\mathbf{U}}}{2} n_{i}\left(n_{i}+1\right)-\tilde{\mu}\left(n_{i}+1\right)-\frac{\tilde{\mathbf{V}}}{2} \tilde{n}_{i}\left(n_{i}+2\right)+\tilde{\mathbf{J}} \phi_{i}^{*} \tilde{\phi}_{i}, \\
\mathbf{H}_{i}^{0}\left|n_{i}-1\right\rangle=E_{n_{i}-1}^{0}\left|n_{i}-1\right\rangle \Rightarrow E_{n_{i}-1}^{0}=\frac{\tilde{\mathbf{U}}}{2}\left(n_{i}-1\right)\left(n_{i}-2\right)-\tilde{\mu}\left(n_{i}-1\right)-\frac{\tilde{\mathbf{V}}}{2} \tilde{n}_{i}\left(n_{i}-2\right)+\tilde{\mathbf{J}} \phi_{i}^{*} \tilde{\phi}_{i} .
\end{gathered}
$$

Substituindo essas energias e a Eq. (C.9) na aproximação da energia total, Eq. (C.7), deduzimos

$$
E_{n} \approx \varepsilon_{n}^{0}+\left|\tilde{\phi}_{i}\right|^{2}\left[\tilde{\mathbf{J}} \frac{\phi_{i}^{*}}{\tilde{\phi}_{i}^{*}}+\tilde{\mathbf{J}}^{2}\left\{\frac{n_{i}+1}{-\tilde{\mathbf{U}} n_{i}+\tilde{\mu}+\tilde{\mathbf{V}} \tilde{n}_{i}}+\frac{n_{i}}{\tilde{\mathbf{U}}\left(n_{i}-1\right)-\tilde{\mu}-\tilde{\mathbf{V}} \tilde{n}_{i}}\right\}\right]
$$

onde $\varepsilon_{n}^{0}=\frac{1}{2} \tilde{\mathbf{U}} n_{i}\left(n_{i}-1\right)-\tilde{\mu} n_{i}-\frac{1}{2} \tilde{\mathbf{V}} \tilde{n}_{i} n_{i}$.

Lembrando que o termo de segundo ordem fornece a TFQ em concordância com a energia livre de Landau para a TFQ. Zerando o coeficiente que acompanha ao parâmetro de ordem da equação anterior, derivamos a equação dos lóbulos

$$
\phi_{i}=\tilde{\phi}_{i} \tilde{\mathbf{J}}\left\{\frac{n_{i}+1}{\tilde{\mathbf{U}} n_{i}-\tilde{\mu}-\tilde{\mathbf{V}} \tilde{n}_{i}}+\frac{n_{i}}{-\tilde{\mathbf{U}}\left(n_{i}-1\right)+\tilde{\mu}+\tilde{\mathbf{V}} \tilde{n}_{i}}\right\}
$$

onde foi tomado o complexo conjugado ao parâmetro de ordem. 
